\title{
Total synthesis of (-)-borrelidin
}

\author{
Tohru Nagamitsu, Daisuke Takano, Takeo Fukuda, Kazuhiko Otoguro, Yoshihiro \\ Harigaya, Isao Kuwajima and Satoshi $\bar{O} m u r a *$
}

\section{Supporting Information}

General Information. All non-aqueous reactions were carried out under an inert atmosphere of argon. Organic solvents were dried over molecular sieves 3A or 4A. Other reagents were commercially available and used without further purification. Optical rotations were measured on a JASCO DIP-1000 digital polarimeter with a sodium lamp. Infrared spectra were recorded on a Horiba FT-210 and JASCO FT/IR-460. ${ }^{1} \mathrm{H}-\mathrm{NMR}$ and ${ }^{13} \mathrm{C}-\mathrm{NMR}$ spectra were recorded on a JEOL JNM-EX270. Ambiguous assignments were resolved on the basis of two-dimensional COSY experiments. High-resolution mass spectra were obtained on JEOL JMS-700 (FAB) and JEOL JMS-AX505HA (EI).

$(2 R, 4 S)-5-A c e t o x y-2,4-d i m e t h y l p e n t a n e-1-o l ~(3)$

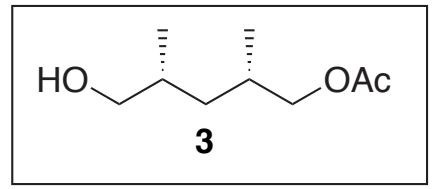

This compound was prepared according to Mori's procedure. (Fujita, K.; Mori, K. Eur. J. Org. Chem. 2001, 66, 493-502.)

(2S,4R)-1-Acetoxy-5-(tert-butyldimethylsilyloxy)-2,4-dimethylpentane (3a)

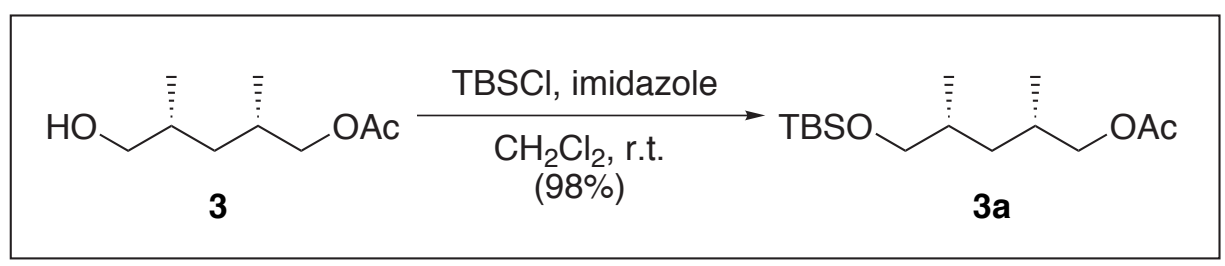

To a solution of $\mathbf{3}(1.01 \mathrm{~g}, 5.83 \mathrm{mmol})$ in $\mathrm{CH}_{2} \mathrm{Cl}_{2}(20 \mathrm{ml})$ were added imidazole $(790 \mathrm{mg}, 11.6$ 
$\mathrm{mmol})$ and $\mathrm{TBSCl}(1.30 \mathrm{~g}, 8.74 \mathrm{mmol})$ at r.t.. The resulting solution was stirred for $30 \mathrm{~min}$ and quenched with water. The aqueous phase was extracted with $\mathrm{CH}_{2} \mathrm{Cl}_{2}$. The combined organic extracts were dried over anhydrous $\mathrm{Na}_{2} \mathrm{SO}_{4}$ and concentrated in vacuo. Flash chromatography (30:1 hexanes/EtOAc) afforded $\mathbf{3 a}(1.64 \mathrm{~g}, 98 \%)$ as a colorless oil.

$[\alpha]_{\mathrm{D}}^{22}=+5.6^{\circ}\left(c=0.24, \mathrm{CHCl}_{3}\right)$; IR $(\mathbf{K B r}) 2956,2931,2892,2888,1743,1251,1238 \mathrm{~cm}^{-1}$; ${ }^{1} \mathbf{H}-$ NMR (270 MHz, $\left.\mathbf{C D C l}_{3}\right) \delta 0.01\left(\mathrm{~s}, 6 \mathrm{H}, \mathrm{CH}_{3} \mathrm{Si}\right), 0.87$ (s, 9H, $\left.\mathrm{CH}_{3} \mathrm{CSi}\right), 0.88(\mathrm{~d}, 3 \mathrm{H}, J=6.6 \mathrm{~Hz}$, $\left.\mathrm{C}_{4}-\mathrm{CH}_{3}\right), 0.90\left(\mathrm{~m}, 1 \mathrm{H}, 1 / 2 \mathrm{C}_{3}-\mathbf{H}\right), 0.92\left(\mathrm{~d}, 3 \mathrm{H}, J=6.6 \mathrm{~Hz}, \mathrm{C}_{2}-\mathrm{CH}_{3}\right), 1.44\left(\mathrm{~m}, 1 \mathrm{H}, 1 / 2 \mathrm{C}_{3}-\mathbf{H}\right), 1.67$ $\left(\mathrm{m}, 1 \mathrm{H}, \mathrm{C}_{4}-\mathbf{H}\right), 1.88\left(\mathrm{~m}, 1 \mathrm{H}, \mathrm{C}_{2}-\mathbf{H}\right), 2.02\left(\mathrm{~s}, 3 \mathrm{H}, \mathbf{C O C H}_{3}\right), 3.34\left(\mathrm{dd}, 1 \mathrm{H}, J=9.6,6.3 \mathrm{~Hz}, 1 / 2 \mathrm{C}_{5^{-}}\right.$ H), $3.41\left(\mathrm{dd}, 1 \mathrm{H}, J=9.6,5.6 \mathrm{~Hz}, 1 / 2 \mathrm{C}_{5}-\mathbf{H}\right), 3.80\left(\mathrm{dd}, 1 \mathrm{H}, J=10.9,6.9 \mathrm{~Hz}, 1 / 2 \mathrm{C}_{1}-\mathbf{H}\right), 3.94(\mathrm{dd}$, $\left.1 \mathrm{H}, J=10.9,5.3 \mathrm{~Hz}, 1 / 2 \mathrm{C}_{1}-\mathbf{H}\right) ;{ }^{13} \mathbf{C}-\mathbf{N M R}\left(\mathbf{6 7 . 5} \mathbf{M H z}, \mathbf{C D C l}_{3}\right) \delta$-5.4, 17.4, 17.8, 18.2, 20.9, 25.9, 30.0, 33.0, 37.4, 68.0, 69.3, 171.2; HRMS [FAB, m-NBA] calcd for $\mathrm{C}_{15} \mathrm{H}_{33} \mathrm{O}_{3} \mathrm{Si}\left[\mathrm{M}+\mathrm{H}^{+}\right]$: 289.2199; found: 289.2193 .

\section{(2S,4R)-5-(tert-Butyldimethylsilyloxy)-2,4-dimethylpentan-1-ol (3b)}

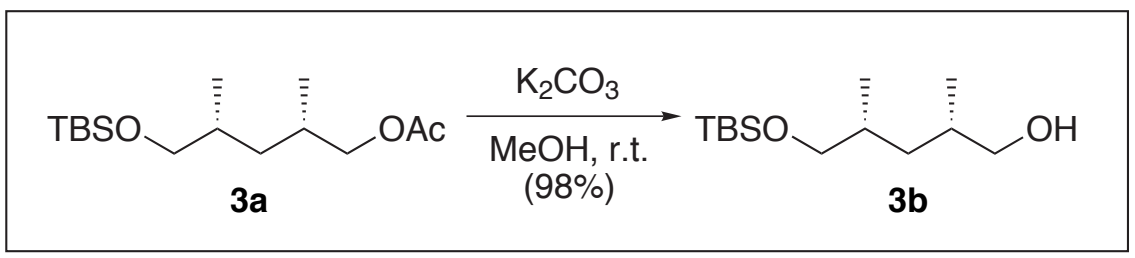

To a stirred solution of 3a $(1.64 \mathrm{~g}, 5.72 \mathrm{mmol})$ in $\mathrm{MeOH}(6 \mathrm{ml})$ was added potassium carbonate ( $870 \mathrm{mg}, 6.29 \mathrm{mmol})$. The reaction was stirred at r.t. for $2 \mathrm{~h}$, and then diluted with water. The aqueous phase was extracted with EtOAc. The combined organic extracts were dried over anhydrous $\mathrm{Na}_{2} \mathrm{SO}_{4}$ and concentrated in vacuo. Flash chromatography (10:1 hexanes/EtOAc) afforded $3 \mathbf{b}(1.47 \mathrm{~g}, 98 \%)$ as a colorless oil.

$[\alpha]_{\mathrm{D}}^{22}=+0.8^{\circ}\left(c=0.32, \mathrm{CHCl}_{3}\right)$; IR (KBr) 3411, 2956, 2929, 2858, 1471, 1255, 1093, $837 \mathrm{~cm}^{-1}$; ${ }^{1} \mathrm{H}-\mathrm{NMR}\left(270 \mathrm{MHz}, \mathbf{C D C l}_{3}\right) \delta 0.03\left(\mathrm{~s}, 6 \mathrm{H}, \mathrm{CH}_{3} \mathrm{Si}\right), 0.88\left(\mathrm{~m}, 1 \mathrm{H}, 1 / 2 \mathrm{C}_{3}-\mathbf{H}\right), 0.89(\mathrm{~s}, 9 \mathrm{H}$, $\left.\mathrm{CH}_{3} \mathrm{CSi}\right), 0.90\left(\mathrm{~d}, 3 \mathrm{H}, J=6.6 \mathrm{~Hz}, \mathrm{CH}_{3}\right), 0.93\left(\mathrm{~d}, 3 \mathrm{H}, J=6.6 \mathrm{~Hz}, \mathrm{CH}_{3}\right), 1.42\left(\mathrm{~m}, 1 \mathrm{H}, 1 / 2 \mathrm{C}_{3}-\mathbf{H}\right)$, $1.70(\mathrm{~m}, 1 \mathrm{H}, \mathbf{C H}), 1.81$ (m, 1H, CH), 3.32-3.52 (m, 4H, CH $\mathbf{C H}_{2}$ ); ${ }^{13} \mathbf{C}-\mathbf{N M R}\left(\mathbf{6 7 . 5} \mathbf{~ M H z}, \mathbf{C D C l}_{3}\right)$ $\delta$-5.4, 17.7, 17.8, 18.3, 25.9, 33.2, 33.3, 37.3, 68.1, 68.3; HRMS [FAB, m-NBA] calcd for $\mathrm{C}_{13} \mathrm{H}_{31} \mathrm{O}_{2} \mathrm{Si}\left[\mathrm{M}+\mathrm{H}^{+}\right]: 247.2093$; found: 247.2079. 
(2R,4S)-5-(tert-Butyldiphenylsilyloxy)-2,4-dimethylpentan-1-ol (3c)

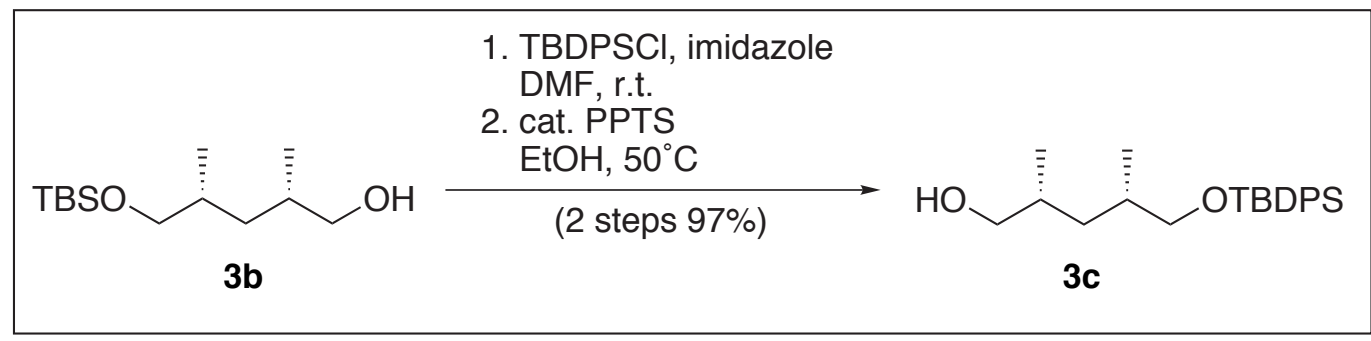

To a solution of $\mathbf{3 b}(3.10 \mathrm{~g}, 12.6 \mathrm{mmol})$ in DMF $(63 \mathrm{ml})$ were added imidazole $(1.70 \mathrm{~g}, 25.2$ mmol) and TBDPSCl $(4.9 \mathrm{ml}, 18.9 \mathrm{mmol})$ at $0^{\circ} \mathrm{C}$. The reaction was allowed to warm to r.t. and stirred for $30 \mathrm{~min}$. After addition of water, the reaction mixture was extracted with EtOAc. The combined organic extracts were dried over anhydrous $\mathrm{Na}_{2} \mathrm{SO}_{4}$ and concentrated in vacuo. Flash chromatography (50:1 hexanes/EtOAc) afforded the corresponding disilyl ether (5.52 g) including unseparable impurities.

To a solution of the disilyl ether in EtOH $(40 \mathrm{ml})$ was added PPTS (1.43 g, $5.70 \mathrm{mmol})$, and the mixture was allowed to warm up to $50^{\circ} \mathrm{C}$. After $7 \mathrm{~h}$, water was added and the aqueous phase was extracted with EtOAc. The combined organic extracts were dried over anhydrous $\mathrm{Na}_{2} \mathrm{SO}_{4}$ and concentrated in vacuo. Flash chromatography (15:1 hexanes/EtOAc) afforded 3c (4.19 g, 2 steps $97 \%)$ as a colorless oil.

$[\alpha]_{\mathrm{D}}{ }^{23}=+1.8^{\circ}\left(c=0.23, \mathrm{CHCl}_{3}\right)$; IR (KBr) 3455, 2931, 2856, 1112, 1091, $702 \mathrm{~cm}^{-1}$; ${ }^{1} \mathbf{H}-\mathbf{N M R}$ $\left(270 \mathbf{~ M H z}, \mathbf{C D C l}_{3}\right) \delta 0.91\left(\mathrm{~d}, 3 \mathrm{H}, J=6.6 \mathrm{~Hz}, \mathrm{CH}_{3}\right), 0.95\left(\mathrm{~m}, 1 \mathrm{H}, 1 / 2 \mathrm{C}_{3}-\mathbf{H}\right), 0.98(\mathrm{~d}, 3 \mathrm{H}, J=6.6$

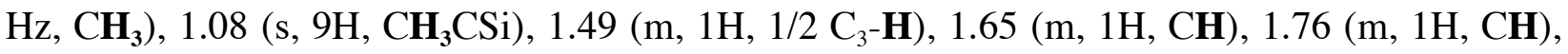
$3.36\left(\mathrm{dd}, 1 \mathrm{H}, J=10.2,6.6 \mathrm{~Hz}, 1 / 2 \mathbf{C H}_{2}\right), 3.45\left(\mathrm{~m}, 1 \mathrm{H}, 1 / 2 \mathbf{C H}_{2}\right), 3.51(\mathrm{dd}, 1 \mathrm{H}, J=9.6,5.3 \mathrm{~Hz}$, 1/2 $\left.\mathbf{C H}_{2}\right), 3.55\left(\mathrm{dd}, 1 \mathrm{H}, J=9.6,5.3 \mathrm{~Hz}, 1 / 2 \mathrm{CH}_{2}\right), 7.35-7.72(\mathrm{~m}, 10 \mathrm{H}, \operatorname{ArH}) ;{ }^{13} \mathbf{C}-\mathbf{N M R}(\mathbf{6 7 . 5}$ MHz, $\mathbf{C D C l}_{3}$ ) $\delta 17.4,17.9,19.3,26.9,33.1,37.1,68.2,68.7,127.6,129.5,134.0,135.6$; HRMS [FAB, m-NBA] calcd for $\mathrm{C}_{23} \mathrm{H}_{35} \mathrm{O}_{2} \mathrm{Si}\left[\mathrm{M}+\mathrm{H}^{+}\right]$: 371.2406; found: 371.2415 .

\section{(2R,4S)-5-(tert-Butyldiphenylsilyloxy)-2,4-dimethylpentanal (4)}

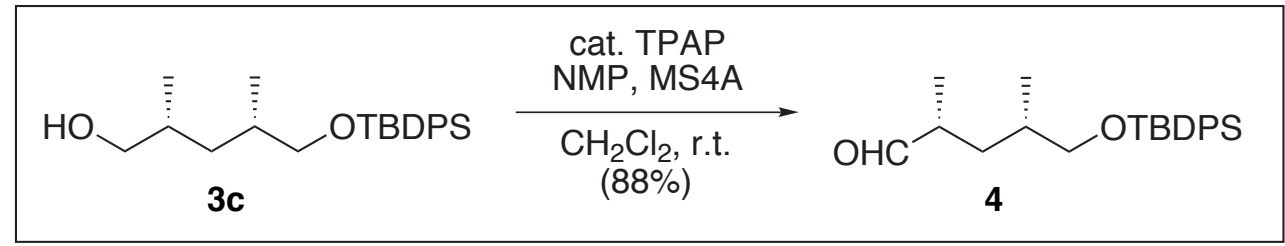

To a solution of $3 \mathbf{c}(89.3 \mathrm{mg}, 0.241 \mathrm{mmol})$ in $\mathrm{CH}_{2} \mathrm{Cl}_{2}(2.5 \mathrm{ml})$ were added dried MS4A $(500 \mathrm{mg})$, 
NMO $(31.1 \mathrm{mg}, 0.265 \mathrm{mmol})$ and TPAP $(4.2 \mathrm{mg}, 12.0 \mu \mathrm{mol})$ at r.t.. The resulting solution was stirred for $30 \mathrm{~min}$ and the reaction mixture was filtered through a silica pad. After evaporation of the filtrate, the residue was purified by flash chromatography (50:1 hexanes/EtOAc) to afford 4 (78.0 $\mathrm{mg}, 88 \%)$ as a colorless oil.

$[\alpha]_{\mathrm{D}}{ }^{23}=-6.3^{\circ}\left(c=0.40, \mathrm{CHCl}_{3}\right)$; IR (KBr) $2960,2931,2858,1727,1427,1112,1089 \mathrm{~cm}^{-1} ;{ }^{1} \mathbf{H}-$ NMR (270 MHz, $\left.\mathbf{C D C l}_{3}\right) \delta 0.94\left(\mathrm{~d}, 3 \mathrm{H}, J=6.6 \mathrm{~Hz}, \mathrm{C}_{4}-\mathrm{CH}_{3}\right), 1.04\left(\mathrm{~d}, 3 \mathrm{H}, J=6.6 \mathrm{~Hz}, \mathrm{C}_{2}-\mathrm{CH}_{3}\right)$, 1.06 (s, 9H, $\left.\mathbf{C H}_{3} \mathrm{CSi}\right), 1.14\left(\mathrm{~m}, 1 \mathrm{H}, 1 / 2 \mathrm{C}_{3}-\mathbf{H}\right), 1.75\left(\mathrm{~m}, 1 \mathrm{H}, \mathrm{C}_{4}-\mathbf{H}\right), 1.90\left(\mathrm{~m}, 1 \mathrm{H}, 1 / 2 \mathrm{C}_{3}-\mathbf{H}\right), 2.39$ $\left(\mathrm{m}, 1 \mathrm{H}, \mathrm{C}_{2}-\mathbf{H}\right), 3.49\left(\mathrm{~d}, 2 \mathrm{H}, J=5.6 \mathrm{~Hz}, \mathrm{C}_{5}-\mathbf{H}\right), 7.34-7.70(\mathrm{~m}, 10 \mathrm{H}, \mathrm{ArH}), 9.54(\mathrm{~d}, 1 \mathrm{H}, J=2.3 \mathrm{~Hz}$, CHO); ${ }^{13}$ C-NMR (67.5 MHz, $\mathbf{C D C l}_{3}$ ) $\delta$ 14.1, 17.2, 19.3, 26.9, 33.3, 34.5, 44.1, 68.4, 127.6, 129.6, 133.7, 135.6, 205.2; HRMS [FAB, m-NBA] calcd for $\mathrm{C}_{23} \mathrm{H}_{32} \mathrm{O}_{2} \mathrm{Si}\left[\mathrm{M}^{+}\right]: 368.2171$; found: 368.2142.

(3S)-1,1-Dibromo-4-(4'-methoxybenzyloxy)-3-methylbut-1-ene (5)

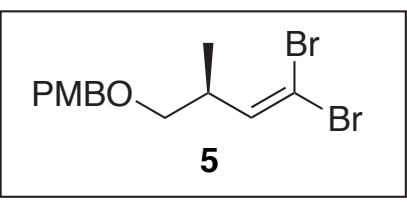

This compound was prepared by using a known method. (Paquette, L. A.; Guevel, R.; Sakamoto, S.; Kim, I. H.; Crawford, J. J. Org. Chem. 2003, 68, 6096-6107.)

\section{$(2 S, 6 R, 8 S)-9-($ tert-Butyldiphenylsilyloxy)-1-(4'-methoxybenzyloxy)-2,6,8-trimethylnona-3-} yne (4a)

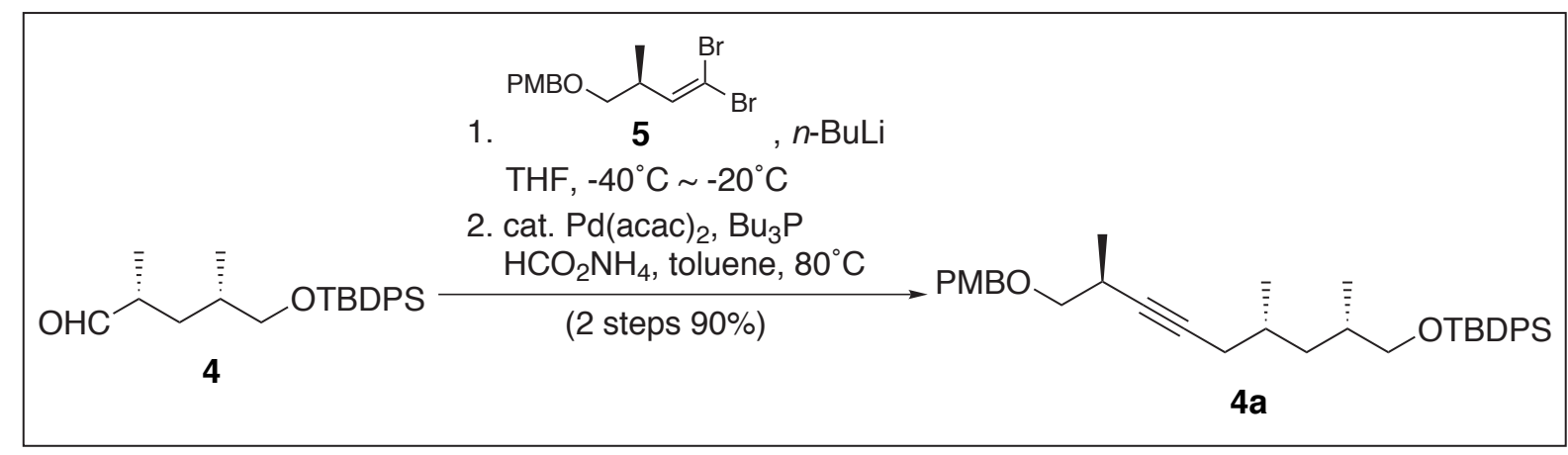

To a solution of $5(83.0 \mathrm{mg}, 0.229 \mathrm{mmol})$ in THF (700 $\mu \mathrm{l})$ was added $n$-BuLi (1.58 M solution in hexane, $290 \mu \mathrm{l}, 0.459 \mathrm{mmol}$ ) at $-78^{\circ} \mathrm{C}$, and the mixture was allowed to warm up to $-40^{\circ} \mathrm{C}$. The reaction was stirred for $1 \mathrm{~h}$, and then treated with a solution of $4(56.3 \mathrm{mg}, 0.153 \mathrm{mmol})$ in THF 
$(700 \mu \mathrm{l})$. The reaction was continued for $1 \mathrm{~h}$ at $-30^{\circ} \mathrm{C}$, and methyl chloroformate $(90 \mu \mathrm{l}, 1.15$ mmol) was added at $-20^{\circ} \mathrm{C}$. The reaction was quenched with sat. aq. $\mathrm{NH}_{4} \mathrm{Cl}$, and the aqueous phase was extracted with EtOAc. The combined organic extracts were dried over anhydrous $\mathrm{Na}_{2} \mathrm{SO}_{4}$ and concentrated in vacuo. Flash chromatography (30:1 hexanes/EtOAc) afforded the corresponding carbonate (100.8 mg) including unseparable impurities.

To a solution of palladium acetylacetonate $(2.3 \mathrm{mg}, 7.65 \mu \mathrm{mol})$ and tributylphosphine $(8 \mu \mathrm{l}, 30.6$ $\mu \mathrm{mol})$ in toluene $(700 \mu \mathrm{l})$ were added a solution of the crude carbonate in toluene $(700 \mu \mathrm{l})$ and ammonium formate $(24.1 \mathrm{mg}, 0.382 \mathrm{mmol})$. The resulting solution was warmed up to $70^{\circ} \mathrm{C}$ and stirred for $1 \mathrm{hr}$. Concentration in vacuo gave a yellow oil, which upon flash chromatography (50:1 hexanes/EtOAc) afforded $\mathbf{4 a}(76,3 \mathrm{mg}, 2$ steps 90\%) as a colorless oil.

$[\alpha]_{\mathrm{D}}{ }^{24}=-3.1^{\circ}\left(c=0.29, \mathrm{CHCl}_{3}\right)$; IR (KBr) 2910, 2898, 2856, 1513, 1459, 1112, 1106, $1091 \mathrm{~cm}^{-1}$; ${ }^{1} \mathbf{H}-N M R\left(270 \mathbf{M H z} \mathbf{C D C l}_{3}\right) \delta 0.95\left(\mathrm{~d}, 6 \mathrm{H}, J=6.6 \mathrm{~Hz}, \mathrm{C}_{6}-\mathrm{CH}_{3}\right.$ and $\left.\mathrm{C}_{8}-\mathrm{CH}_{3}\right), 0.98(\mathrm{~m}, 1 \mathrm{H}, 1 / 2$ $\left.\mathrm{C}_{7}-\mathbf{H}\right), 1.08\left(\mathrm{~s}, 9 \mathrm{H}, \mathrm{CH}_{3} \mathrm{CSi}\right), 1.17\left(\mathrm{~d}, 3 \mathrm{H}, J=6.9 \mathrm{~Hz}, \mathrm{C}_{2}-\mathrm{CH}_{3}\right), 1.46\left(\mathrm{~m}, 1 \mathrm{H}, 1 / 2 \mathrm{C}_{7}-\mathbf{H}\right), 1.68(\mathrm{~m}$, $\left.1 \mathrm{H}, \mathrm{C}_{6}-\mathbf{H}\right), 1.75\left(\mathrm{~m}, 1 \mathrm{H}, \mathrm{C}_{8}-\mathbf{H}\right), 1.97$ (ddd, 1H, $\left.J=16.2,7.3,1.7 \mathrm{~Hz}, 1 / 2 \mathrm{C}_{5}-\mathbf{H}\right), 2.15$ (ddd, 1H, $J$ $\left.=16.5,4.9,1.7 \mathrm{~Hz}, 1 / 2 \mathrm{C}_{5}-\mathbf{H}\right), 2.70\left(\mathrm{~m}, 1 \mathrm{H}, \mathrm{C}_{2}-\mathbf{H}\right), 3.30\left(\mathrm{~m}, 1 \mathrm{H}, 1 / 2 \mathrm{C}_{1}-\mathbf{H}\right), 3.48\left(\mathrm{~m}, 3 \mathrm{H}, \mathrm{C}_{9}-\mathbf{H}\right.$ and 1/2 $\left.\mathrm{C}_{1}-\mathbf{H}\right), 3.81\left(\mathrm{~s}, 3 \mathrm{H}, \mathrm{OCH}_{3}\right), 4.48\left(\mathrm{~s}, 2 \mathrm{H}, \mathrm{CH}_{2} \mathrm{Ph}\right), 6.89(\mathrm{~d}, 2 \mathrm{H}, J=8.6 \mathrm{~Hz}, \mathrm{ArH}), 7.28(\mathrm{~d}$, $2 \mathrm{H}, J=8.6 \mathrm{~Hz}, \operatorname{ArH}), 7.37-7.71(\mathrm{~m}, 10 \mathrm{H}, \operatorname{ArH}) ;{ }^{13} \mathbf{C}-\mathbf{N M R}\left(67.5 \mathbf{M H z}, \mathbf{C D C l}_{3}\right) \delta ; 17.5,18.3$, 19.3, 20.1, 25.9, 26.7, 26.9, 30.1, 33.2, 40.1, 55.2, 69.0, 72.6, 74.3, 79.7, 83.0, 113.7, 127.5, 129.2, 129.5, 130.5, 134.0, 135.6, 159.1 HRMS [FAB, m-NBA] calcd for $\mathrm{C}_{36} \mathrm{H}_{48} \mathrm{O}_{3} \mathrm{Si}\left[\mathrm{M}+\mathrm{H}^{+}\right]$: 556.3372; found: 556.3364 .

(2S,6R,8S)-9-(tert-Butyldiphenylsilyloxy)-2,6,8-trimethylnona-3-yn-1-ol (6)

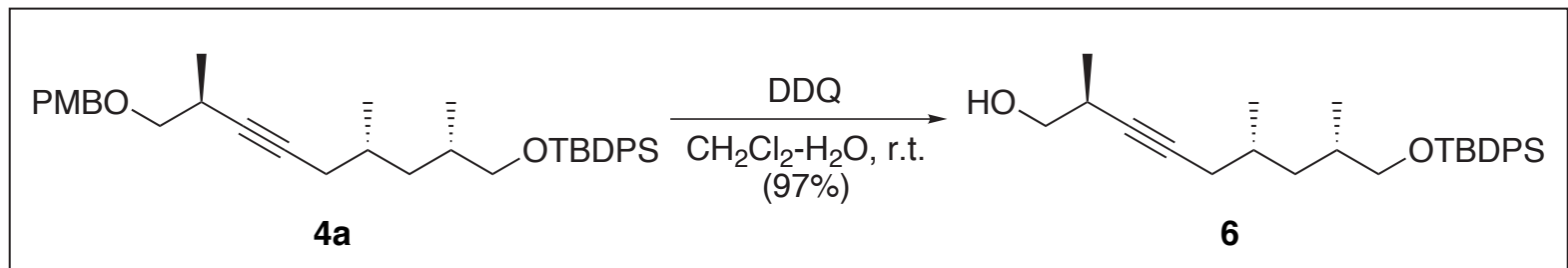

To a solution of $4 \mathbf{a}(648.6 \mathrm{mg}, 1.16 \mathrm{mmol})$ in $\mathrm{CH}_{2} \mathrm{Cl}_{2}(12 \mathrm{ml})$ and $\mathrm{H}_{2} \mathrm{O}(1.2 \mathrm{ml})$ was added DDQ $(344.0 \mathrm{mg}, 1.51 \mathrm{mmol})$ at r.t.. The reaction mixture was stirred for $30 \mathrm{~min}$, and then diluted with water. The aqueous phase was extracted with EtOAc. The combined organic extracts were dried over anhydrous $\mathrm{Na}_{2} \mathrm{SO}_{4}$ and concentrated in vacuo. Flash chromatography (30:1 hexanes/EtOAc) afforded 6 (494.1 mg, 97\%) as a colorless oil. 
$[\alpha]_{\mathrm{D}}{ }^{24}=-11.6^{\circ}\left(c=0.26, \mathrm{CHCl}_{3}\right)$; IR (KBr) 3644, 2964, 2960, 2931, 2325, 1112, $761 \mathrm{~cm}^{-1} ;{ }^{1} \mathbf{H}-$ NMR (270 MHz, $\left.\mathbf{C D C l}_{3}\right) \delta 0.96\left(\mathrm{~d}, 6 \mathrm{H}, J=6.6 \mathrm{~Hz}, \mathrm{C}_{6}-\mathrm{CH}_{3}\right.$ and $\left.\mathrm{C}_{8}-\mathrm{CH}_{3}\right), 0.98\left(\mathrm{~m}, 1 \mathrm{H}, 1 / 2 \mathrm{C}_{7^{-}}\right.$ H), 1.08 (s, 9H, CH $\mathbf{C H S i}_{3}, 1.14\left(\mathrm{~d}, 3 \mathrm{H}, J=6.9 \mathrm{~Hz}, \mathrm{C}_{2}-\mathrm{CH}_{3}\right), 1.47\left(\mathrm{~m}, 1 \mathrm{H}, 1 / 2 \mathrm{C}_{7}-\mathbf{H}\right), 1.70(\mathrm{~m}, 1 \mathrm{H}$, $\left.\mathrm{C}_{6}-\mathbf{H}\right), 1.75\left(\mathrm{~m}, 1 \mathrm{H}, \mathrm{C}_{8}-\mathbf{H}\right), 1.98\left(\mathrm{ddd}, 1 \mathrm{H}, J=16.5,6.9,1.7 \mathrm{~Hz}, 1 / 2 \mathrm{C}_{5}-\mathbf{H}\right), 2.17$ (ddd, 1H, $J=$ 16.1, 5.0, $\left.1.7 \mathrm{~Hz}, 1 / 2 \mathrm{C}_{5}-\mathbf{H}\right), 2.64\left(\mathrm{~m}, 1 \mathrm{H}, \mathrm{C}_{2}-\mathbf{H}\right), 3.49\left(\mathrm{~m}, 4 \mathrm{H}, \mathrm{C}_{1}-\mathbf{H}\right.$ and $\left.\mathrm{C}_{9}-\mathbf{H}\right)$, 7.37-7.71 (m, 10H, ArH); ${ }^{13} \mathbf{C}-\mathbf{N M R}(67.5$ MHz, $\mathbf{C D C l}$ ) $\delta 17.3,17.5,19.3,20.2,25.8,26.9,29.5,30.0,33.2$, 40.1, 67.1, 68.9, 81.1, 82.4, 127.6, 129.5, 134.0, 135.6; HRMS [FAB, m-NBA] calcd for $\mathrm{C}_{28} \mathrm{H}_{40} \mathrm{O}_{2} \mathrm{SiNa}\left[\mathrm{M}+\mathrm{Na}^{+}\right]$: 459.2695; found: 459.2677 .

\section{(2S,6R,8S)-9-(tert-Butyldiphenylsilyloxy)-2,4,6,8-tetramethylnona-3Z-en-1-ol (7)}

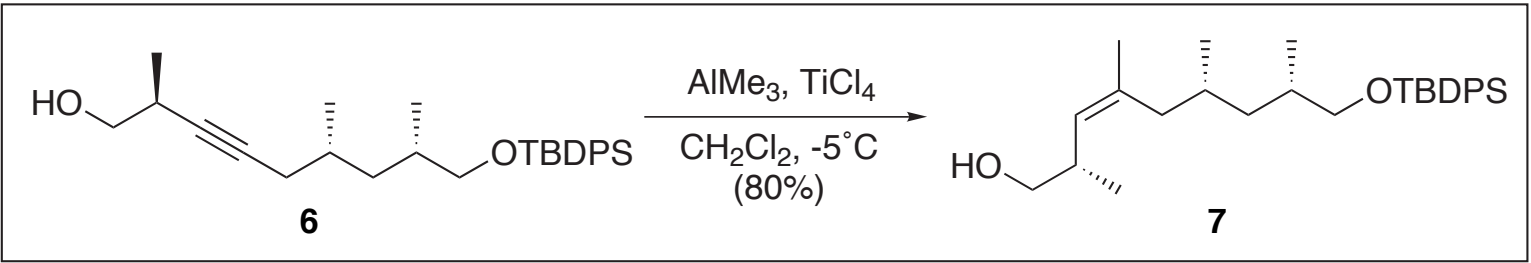

To a solution of trimethylaluminium (1.0 M solution in hexanes, $6.2 \mathrm{ml}, 6.20 \mathrm{mmol})$ was added a solution of $6(672.5 \mathrm{mg}, 1.54 \mathrm{mmol})$ in $\mathrm{CH}_{2} \mathrm{Cl}_{2}(3 \mathrm{ml})$ at $0^{\circ} \mathrm{C}$. The mixture was allowed to warm to r.t., stirred for $20 \mathrm{~min}$, and then cooled to $-15^{\circ} \mathrm{C}$. The resulting solution was treated with titanium tetrachloride (1.0 M solution in $\mathrm{CH}_{2} \mathrm{Cl}_{2}, 3.1 \mathrm{ml}, 3.10 \mathrm{mmol}$ ) and continued for $15 \mathrm{~min}$. The reaction was cooled to $-40^{\circ} \mathrm{C}$ and then cautiously quenched with ice-cooled $\mathrm{MeOH}(3 \mathrm{ml})$ followed by Celite ${ }^{\circledR}(10 \mathrm{~g})$ and $\mathrm{Na}_{2} \mathrm{SO}_{4} \cdot 10 \mathrm{H}_{2} \mathrm{O}(10 \mathrm{~g})$. The mixuture was allowed to warm to r.t. and stirred for $2 \mathrm{hr}$. The mixture was filtered through Celite ${ }^{\circledR}$ and the filtrate was concentrated in vacuo. The residue was purified by flash chromatography (40:1 hexanes/EtOAc) to afford 7 (557.1 $\mathrm{mg}, 80 \%)$ as a colorless oil.

$[\alpha]_{\mathrm{D}}{ }^{24}=-9.1^{\circ}\left(c=0.17, \mathrm{CHCl}_{3}\right)$; IR (KBr) 3444, 2960, 2931, 2896, 2869, 1473, 1459, 1450, 1427, $1105 \mathrm{~cm}^{-1}$; ${ }^{1} \mathbf{H}-\mathbf{N M R}\left(\mathbf{2 7 0} \mathbf{M H z}, \mathbf{C D C l}_{3}\right) \delta 0.77\left(\mathrm{~d}, 3 \mathrm{H}, J=6.3 \mathrm{~Hz}, \mathrm{C}_{6}-\mathrm{CH}_{3}\right), 0.90(\mathrm{~d}, 3 \mathrm{H}, J$ $\left.=6.6 \mathrm{~Hz}, \mathrm{C}_{2}-\mathrm{CH}_{3}\right), 0.95\left(\mathrm{~d}, 3 \mathrm{H}, J=6.6 \mathrm{~Hz}, \mathrm{C}_{8}-\mathrm{CH}_{3}\right), 0.98\left(\mathrm{~m}, 1 \mathrm{H}, 1 / 2 \mathrm{C}_{7}-\mathbf{H}\right), 1.06(\mathrm{~s}, 9 \mathrm{H}$, $\mathrm{CH}_{3} \mathrm{CSi}$ ), 1.34 (m, 1H, 1/2 C $\left.-\mathbf{H}\right), 1.66$ (s, 3H, $\left.\mathrm{C}_{4}-\mathrm{CH}_{3}\right), 1.70\left(\mathrm{~m}, 1 \mathrm{H}, \mathrm{C}_{6}-\mathbf{H}\right), 1.79\left(\mathrm{~m}, 1 \mathrm{H}, \mathrm{C}_{8}-\mathbf{H}\right)$, $1.90\left(\mathrm{~m}, 2 \mathrm{H}, \mathrm{C}_{5}-\mathbf{H}\right), 2.62\left(\mathrm{~m}, 1 \mathrm{H}, \mathrm{C}_{2}-\mathbf{H}\right), 3.28\left(\mathrm{dd}, 1 \mathrm{H}, J=10.2,8.2 \mathrm{~Hz}, 1 / 2 \mathrm{C}_{1}-\mathbf{H}\right), 3.43(\mathrm{~m}, 2 \mathrm{H}$, $1 / 2 \mathrm{C}_{1}-\mathbf{H}$ and $\left.1 / 2 \mathrm{C}_{9}-\mathbf{H}\right), 3.52\left(\mathrm{dd}, 1 \mathrm{H}, J=9.8,5.1 \mathrm{~Hz}, 1 / 2 \mathrm{C}_{9}-\mathbf{H}\right), 4.93\left(\mathrm{~d}, 1 \mathrm{H}, J=9.6 \mathrm{~Hz}, \mathrm{C}_{3}-\mathbf{H}\right)$,

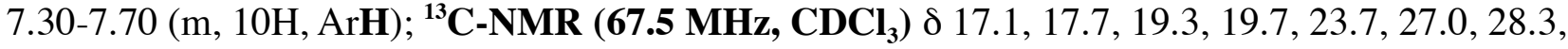
$33.2,35.2,39.5,41.5,67.9,68.9,127.5,128.8,129.5,134.0,135.5,137.0$; HRMS [FAB, m- 
NBA] calcd for $\mathrm{C}_{29} \mathrm{H}_{44} \mathrm{O}_{2} \mathrm{SiNa}\left[\mathrm{M}+\mathrm{Na}^{+}\right]$: 475.3008; found: 475.3022 .

The stereochemistry of C8-9 olefin was determined by NOE experiment.

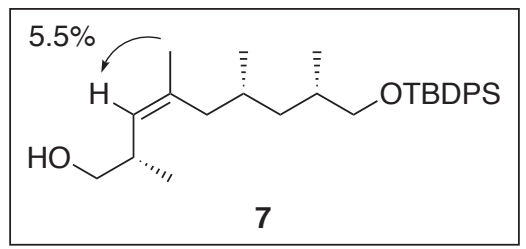

NOE experiment of 7

$(2 S, 4 R, 6 S, 8 S)-9-($ tert-Butyldiphenylsilyloxy)-2,4,6,8-tetramethylnonan-1-ol (8)

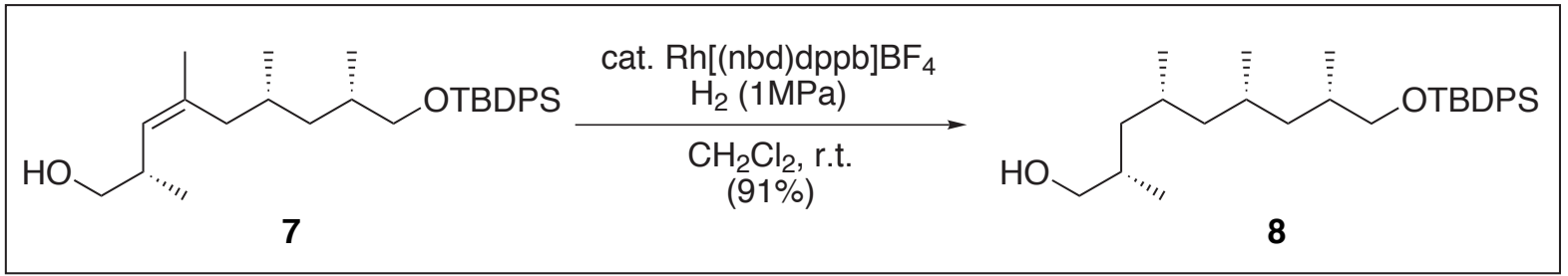

A solution of 7 (941.3 mg, $2.08 \mathrm{mmol})$ and $\mathrm{Rh}[(\mathrm{nbd}) \mathrm{dppb}] \mathrm{BF}_{4}(147.5 \mathrm{mg}, 0.208 \mathrm{mmol})$ in $\mathrm{CH}_{2} \mathrm{Cl}_{2}$ $(4 \mathrm{ml})$ was stirred under $1 \mathrm{Mpa}$ of $\mathrm{H}_{2}$ gas for $2 \mathrm{hr}$. The resulting solution was filtered through a short plug of silica gel and concentrated in vacuo. The residue was purified by flash chromatography (40:1 hexanes/EtOAc) to afford $8(928.5 \mathrm{mg}, 91 \%)$ as a colorless oil. $[\alpha]_{\mathrm{D}}{ }^{25}=-16.6^{\circ}\left(c=0.15, \mathrm{CHCl}_{3}\right)$; IR (KBr) 3425, 2958, 2929, 2858, 1461, 1427, 1112, $1081 \mathrm{~cm}^{-}$ ${ }^{1}$; ${ }^{1} \mathbf{H}-\mathbf{N M R}\left(270 \mathbf{M H z} \mathbf{C D C l}_{3}\right) \delta 0.83\left(\mathrm{~d}, 3 \mathrm{H}, J=6.3 \mathrm{~Hz}, \mathrm{CH}_{3}\right), 0.84\left(\mathrm{~d}, 3 \mathrm{H}, J=6.6 \mathrm{~Hz}, \mathrm{CH}_{3}\right)$, $0.85\left(\mathrm{~m}, 1 \mathrm{H}, 1 / 2 \mathbf{C H}_{2}\right), 0.89\left(\mathrm{~d}, 3 \mathrm{H}, J=6.9 \mathrm{~Hz}, \mathrm{CH}_{3}\right), 0.93\left(\mathrm{~m}, 1 \mathrm{H}, 1 / 2 \mathrm{CH}_{2}\right), 0.95$ (d, $J=6.6 \mathrm{~Hz}$,

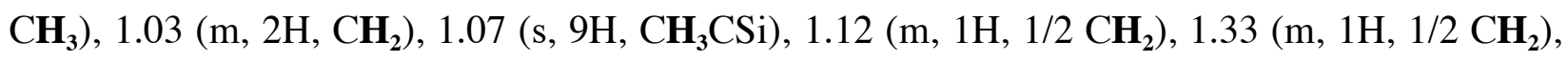
1.52 (m, 1H, CH), 1.57 (m, 1H, CH), 1.70 (m 2H, CH x 2), 3.36-3.59 (m, 4H, CH x 2), 7.30$7.74(\mathrm{~m}, 10 \mathrm{H}, \mathrm{ArH}) ;{ }^{13} \mathbf{C}-\mathbf{N M R}\left(\mathbf{6 7 . 5} \mathbf{M H z}, \mathbf{C D C l}_{3}\right) \delta 16.0,18.0,19.2,20.2,20.5,26.8,27.0$, 27.4 33.1, 33.2, 39.9, 41.5, 46.1, 68.8, 69.2, 127.5, 129.4, 134.0, 135.5; HRMS [FAB, m-NBA] calcd for $\mathrm{C}_{29} \mathrm{H}_{46} \mathrm{O}_{2} \mathrm{SiNa}\left[\mathrm{M}+\mathrm{Na}^{+}\right]$: 477.3164; found: 477.3266 . 
$(2 S, 4 S, 6 R, 8 S)$-1-(tert-Butyldiphenylsilyloxy)-2,4,6,8-tetramethyl-9-(tetrahydropyran-2'yloxy)nonane $(8 a)$

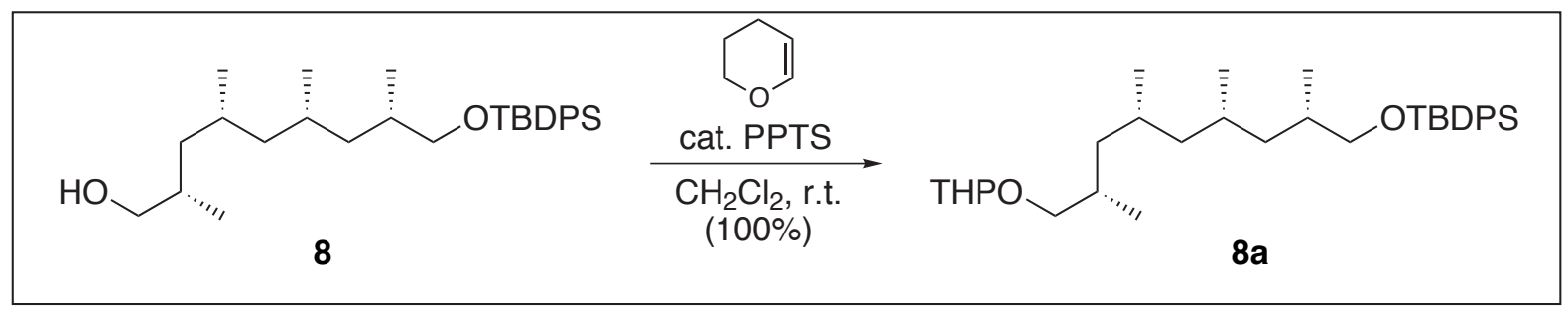

To a solution of $8(928.5 \mathrm{mg}, 2.05 \mathrm{mmol})$ in $\mathrm{CH}_{2} \mathrm{Cl}_{2}(20 \mathrm{ml})$ were added dihydropyrane $(1.85 \mathrm{ml}$, $20.5 \mathrm{mmol}$ ) and PPTS (51 mg, $0.205 \mathrm{mmol})$. The resulting solution was stirred at r.t. for $2 \mathrm{hr}$. The reaction was quenched with sat. aq. $\mathrm{NaHCO}_{3}$ solution and the aqueous phase was extracted with $\mathrm{CH}_{2} \mathrm{Cl}_{2}$. The combined organic extracts were dried over anhydrous $\mathrm{Na}_{2} \mathrm{SO}_{4}$ and concentrated in vacuo. Flash chromatography (50:1 hexanes/EtOAc) afforded 8a (1.10 g, 100\%) as a colorless oil.

$[\alpha]_{\mathrm{D}}{ }^{25}=-10.5^{\circ}\left(c=0.19, \mathrm{CHCl}_{3}\right)$; IR (KBr) 3450, 2956, 2929, 2366, 1473, 1461, $1079 \mathrm{~cm}^{-1} ;{ }^{1} \mathbf{H}-$ NMR (270 MHz, CDCl $)_{3} \delta 0.82\left(\mathrm{~d}, 3 \mathrm{H}, J=6.6 \mathrm{~Hz}, \mathrm{CH}_{3}\right), 0.83\left(\mathrm{~d}, 3 \mathrm{H}, J=6.6 \mathrm{~Hz}, \mathrm{CH}_{3}\right), 0.84(\mathrm{~m}$, $\left.1 \mathrm{H}, 1 / 2 \mathbf{C H}_{2}\right), 0.91\left(\mathrm{~d}, 3 \mathrm{H}, J=6.3 \mathrm{~Hz}, \mathbf{C H}_{3}\right), 0.92\left(\mathrm{~m}, 1 \mathrm{H}, 1 / 2 \mathbf{C H}_{2}\right), 0.94(\mathrm{~d}, 3 \mathrm{H}, J=6.6 \mathrm{~Hz}$,

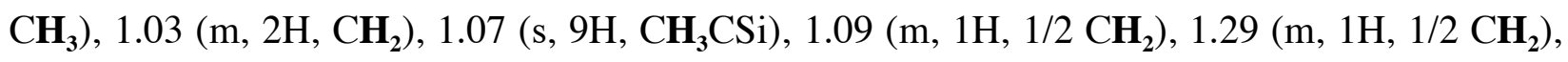
1.47-1.80 (m, 10H, THP(6H) and CH x 4), 3.17 (m, 1H, THP(1H)), 3.39-3.61 (m, 4H, 1/2 CH $\mathrm{CH}_{2}$ and THP(1H)), $3.87\left(\mathrm{~m}, 1 \mathrm{H}, 1 / 2 \mathbf{C H}_{2}\right), 4.58(\mathrm{~m}, 1 \mathrm{H}, \mathrm{THP}(1 \mathrm{H})), 7.35-7.71(\mathrm{~m}, 10 \mathrm{H}, \operatorname{ArH})$; ${ }^{13}$ C-NMR (67.5 MHz, $\mathbf{C D C l}_{3}$ ) $\delta 16.7,16.8,17.9,18.0,19.3,19.5,19.6,20.3,20.6,20.7,25.6$, 26.8, 27.0, 27.1, 27.4, 30.7, 30.8, 30.9, 33.1, 40.6, 41.4, 46.1, 62.0, 62.2, 68.8, 73.8, 74.0, 98.6, 99.0, 127.5, 129.5, 134.1, 135.6; HRMS [FAB, m-NBA] calcd for $\mathrm{C}_{34} \mathrm{H}_{54} \mathrm{O}_{3} \mathrm{SiNa}\left[\mathrm{M}+\mathrm{Na}^{+}\right]$: 561.3739; found: 561.3741.

\section{$(2 S, 4 S, 6 R, 8 S)-2,4,6,8$-Tetramethyl-9-(tetrahydropyran-2'-yloxy)nonan-1-ol (8b)}

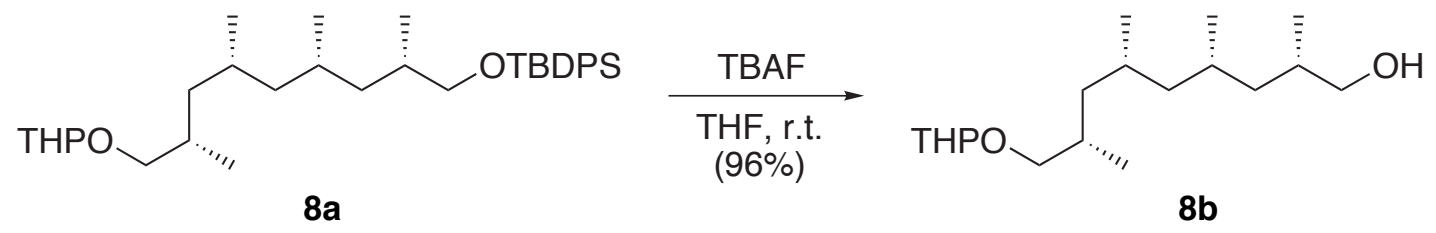

To a stirred solution of $\mathbf{8 a}(1.10 \mathrm{~g}, 2.05 \mathrm{mmol})$ in THF $(20 \mathrm{ml})$ was added TBAF (1.0 M solution 
in THF, $5 \mathrm{ml}, 5.00 \mathrm{mmol}$ ). The resulting solution was stirred for $3 \mathrm{hr}$, quenched with sat. aq. $\mathrm{NH}_{4} \mathrm{Cl}$ solution, and extracted with EtOAc. The combined organic extracts were dried over anhydrous $\mathrm{Na}_{2} \mathrm{SO}_{4}$ and concentrated in vacuo. Flash chromatography (10:1 hexanes/EtOAc) afforded $\mathbf{8 b}$ (590.3 $\mathrm{mg}, 96 \%)$ as a colorless oil.

$[\alpha]_{\mathrm{D}}^{25}=-13.7^{\circ}\left(c=0.29, \mathrm{CHCl}_{3}\right)$; IR (KBr) 3422, 2957, 2922, 2871, 2359, 1457, 1377, 1261, 1201, $1118 \mathrm{~cm}^{-1}$; ${ }^{1} \mathbf{H}-\mathbf{N M R}\left(\mathbf{2 7 0} \mathbf{M H z}, \mathbf{C D C l}_{3}\right) \delta 0.81\left(\mathrm{~d}, 3 \mathrm{H}, J=6.9 \mathrm{~Hz}, \mathrm{CH}_{3}\right), 0.84(\mathrm{~d}, 3 \mathrm{H}, J=$

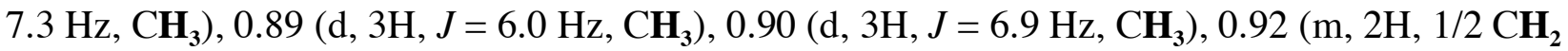
x 2), 1.01-1.32 (m, 4H, 1/2 $\mathbf{C H}_{2} \times 2$ and $\left.\mathbf{C H}_{2}\right), 1.48-1.86(\mathrm{~m}, 10 \mathrm{H}, \mathrm{CH} \times 4$ and THP(6H)), $3.15(\mathrm{~m}$, 1H, THP(1H)), $3.34\left(\mathrm{dd}, 1 \mathrm{H}, J=10.2,6.9 \mathrm{~Hz}, 1 / 2 \mathrm{CH}_{2}\right), 3.41-3.58(\mathrm{~m}, 3 \mathrm{H}, \mathrm{THP}(1 \mathrm{H})$ and $1 / 2$ $\left.\mathrm{CH}_{2} \times 2\right), 3.84\left(\mathrm{~m}, 1 \mathrm{H}, 1 / 2 \mathbf{C H}_{2}\right), 4.54(\mathrm{~m}, 1 \mathrm{H}, \mathrm{THP}(1 \mathrm{H})) ;{ }^{13} \mathbf{C}-\mathbf{N M R}\left(67.5 \mathbf{M H z} \mathbf{C D C l}_{3}\right)$ 16.7, $16.8,17.4,17.5,19.4,19.6,20.3,20.6,20.8,25.5,27.1,27.2,27.4,30.6,30.7,30.8,33.0,40.5$, 41.3, 45.8, 62.0, 68.1, 68.2, 73.6, 73.9, 98.6, 99.0; HRMS [FAB, m-NBA] calcd for $\mathrm{C}_{18} \mathrm{H}_{37} \mathrm{O}_{3}$ $\left[\mathrm{M}+\mathrm{H}^{+}\right]:$301.2742; found: 301.2746.

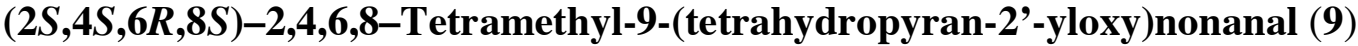

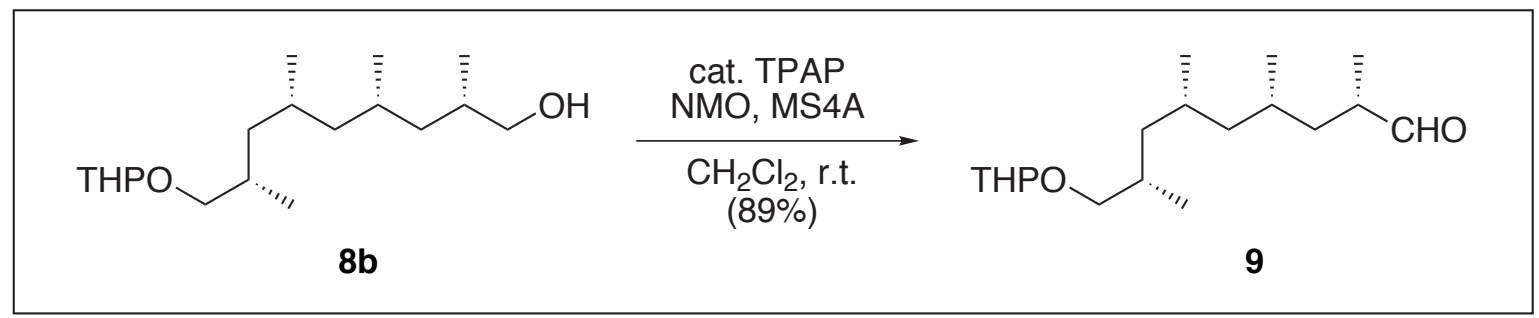

To a solution of $\mathbf{8 b}(388.6 \mathrm{mg}, 1.30 \mathrm{mmol})$ in $\mathrm{CH}_{2} \mathrm{Cl}_{2}(13 \mathrm{ml})$ were added MS4A $(1 \mathrm{~g})$, NMO (321 mg, $2.70 \mathrm{mmol})$ and TPAP $(24.0 \mathrm{mg}, 68.5 \mu \mathrm{mol})$. The reaction was stirred at r.t. for $30 \mathrm{~min}$, and then filtered through a silica pad. After evaporation of the filtrate, the residue was purified by flash chromatography (40:1 hexanes/EtOAc) to afford 9 (343.6 mg, 89\%) as a colorless oil. $[\alpha]_{\mathrm{D}}^{25}=+1.0^{\circ}\left(c=0.14, \mathrm{CHCl}_{3}\right)$; IR (KBr) 2955, 2873, 1734, 1488, 1457, 1261, $1031 \mathrm{~cm}^{-1}$; ${ }^{1} \mathbf{H}-$ NMR (270 MHz, CDCl $) \delta 0.81\left(\mathrm{~d}, 3 \mathrm{H}, J=6.3 \mathrm{~Hz}, \mathrm{CH}_{3}\right), 0.87\left(\mathrm{~d}, 6 \mathrm{H}, J=6.3 \mathrm{~Hz}, \mathrm{CH}_{3} \mathrm{x} 2\right)$, 1.00-1.07 (m, 5H, $\mathbf{C H}_{2} \times 2$ and 1/2 $\left.\mathbf{C H}_{2}\right), 1.16\left(\mathrm{~d}, 3 \mathrm{H}, J=6.9 \mathrm{~Hz}, \mathrm{CH}_{3}\right), 1.47-1.87(\mathrm{~m}, 10 \mathrm{H}$, THP(6H), CH x 3 and 1/2 CH $), 2.56$ (m, 1H, CH), 3.16 (m, 1H, THP(1H)), 3.40-3.58 (m, 2H, 1/2 $\mathrm{CH}_{2}$ and THP(1H)), $3.85\left(\mathrm{~m}, 1 \mathrm{H}, 1 / 2 \mathrm{CH}_{2}\right), 4.56(\mathrm{~m}, 1 \mathrm{H}, \mathrm{THP}(1 \mathrm{H})), 9.57(\mathrm{~d}, 1 \mathrm{H}, J=2.3 \mathrm{~Hz}$, CHO); ${ }^{13} \mathbf{C}-\mathbf{N M R}\left(67.5 \mathbf{M H z} \mathbf{C D C l}_{3}\right) \delta 16.8,16.9,18.0,19.4,19.6,19.8,20.1,25.5,27.0,27.1$, 28.0, 30.6, 30.7, 30.8, 37.2, 40.9, 41.1, 45.9, 62.0, 62.2, 73.6, 73.9, 98.6, 99.0, 182.7; HRMS 
[FAB, m-NBA] calcd for $\mathrm{C}_{18} \mathrm{H}_{34} \mathrm{O}_{3} \mathrm{Na}\left[\mathrm{M}+\mathrm{Na}^{+}\right]: 321.2405$; found: 321.2391 .

(3'S,4R,4'S,6'S,8'R,10'S)-4-Benzyl-3-[3'-hydroxy-4',6',8',10'-tetramethyl-11'(tetrahydropyran-2"-yloxy)undecanoyl]-2-oxazolidinone (9a)

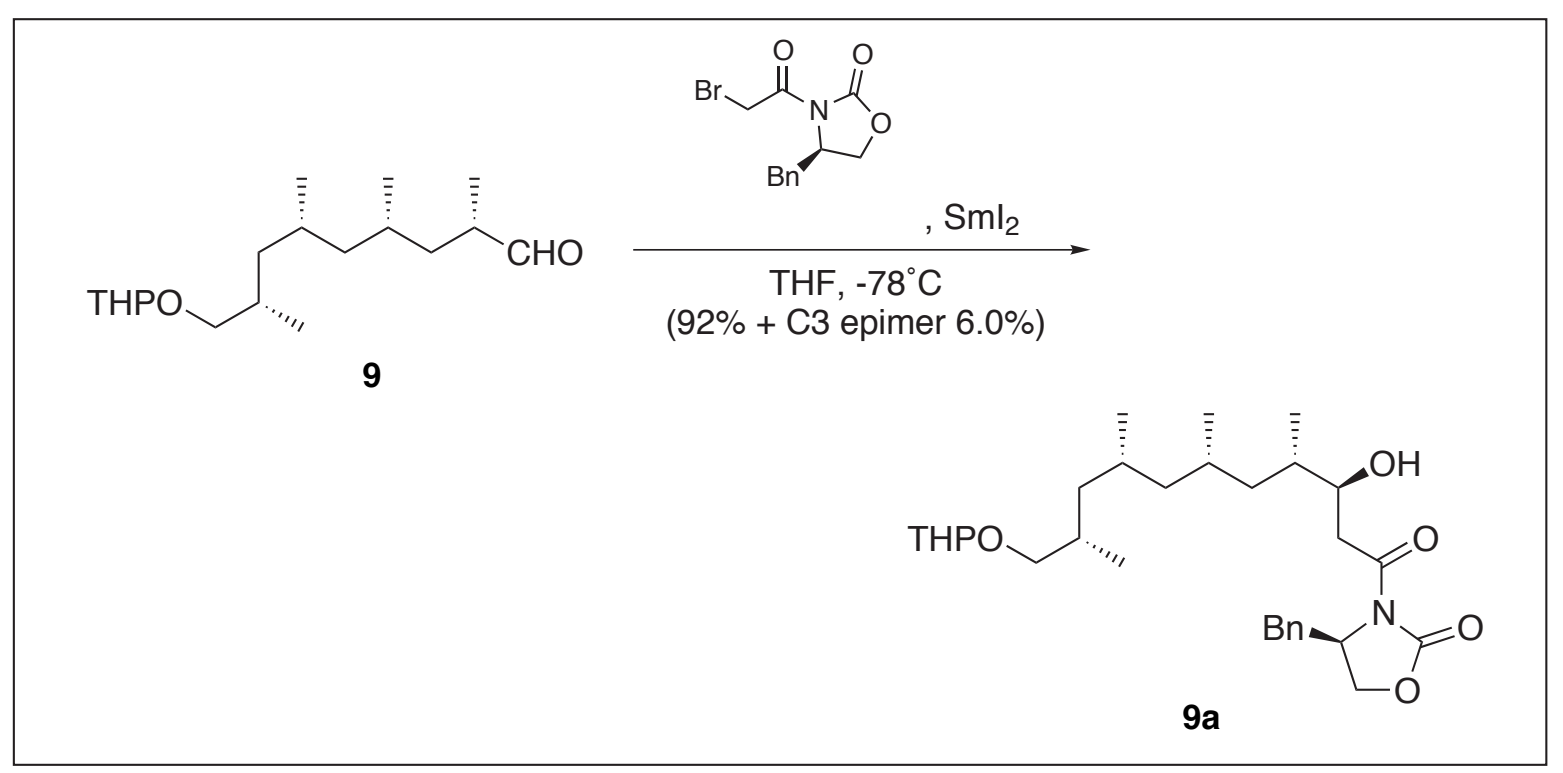

To a solution of samarium iodide $(0.1 \mathrm{M}$ solution in THF, $58.0 \mathrm{ml}, 5.80 \mathrm{mmol})$ were added 9 (343.6 mg, $1.15 \mathrm{mmol}$ ) and ( $R$ )-4-benzyl-3-bromoacetyl-2-oxazolidinone (376.0 mg, $1.26 \mathrm{mmol}$ ) in THF ( $11 \mathrm{ml})$ at $-78^{\circ} \mathrm{C}$. The reaction was stirred for $20 \mathrm{~min}$ and then treated with hexane (100 $\mathrm{ml}$ ) followed by silica gel $(50 \mathrm{~g})$. The mixture was allowed to warm to r.t. and stirred for $30 \mathrm{~min}$. The mixture was filtered through a short plug of silica gel and concentrated in vacuo. The residue was purified by flash chromatography (5:1 hexanes/EtOAc) to afford 9a $(550.7 \mathrm{mg}, 92 \%)$ along with its epimer ( $40.0 \mathrm{mg}, 6.2 \%$ ).

$[\alpha]_{\mathrm{D}}{ }^{26}=-43.8^{\circ}\left(c=0.25, \mathrm{CHCl}_{3}\right) ; \mathbf{I R}(\mathbf{K B r}) 3566,2955,1785,1698,1456,1386,1199,1030 \mathrm{~cm}^{-}$ '; ${ }^{1} \mathbf{H}-\mathbf{N M R}\left(270 \mathrm{MHz}, \mathbf{C D C l}_{3}\right) \delta 0.85\left(\mathrm{~d}, 3 \mathrm{H}, J=5.9 \mathrm{~Hz}, \mathbf{C H}_{3}\right), 0.87\left(\mathrm{~d}, 3 \mathrm{H}, J=5.3 \mathrm{~Hz}, \mathrm{CH}_{3}\right)$, $0.89\left(\mathrm{~d}, 3 \mathrm{H}, J=5.0 \mathrm{~Hz}, \mathrm{CH}_{3}\right), 0.93\left(\mathrm{~d}, 3 \mathrm{H}, J=6.3 \mathrm{~Hz}, \mathrm{CH}_{3}\right), 0.98-1.14\left(\mathrm{~m}, 3 \mathrm{H}, \mathrm{CH}_{2}\right.$ and $1 / 2$ $\left.\mathrm{CH}_{2}\right), 1.25\left(\mathrm{~m}, 2 \mathrm{H}, \mathbf{C H}_{2}\right), 1.42-174\left(\mathrm{~m}, 10 \mathrm{H}, \mathrm{THP}(6 \mathrm{H}), \mathrm{CHx} 3\right.$ and $\left.1 / 2 \mathbf{C H}_{2}\right), 1.83(\mathrm{~m}, 1 \mathrm{H}, \mathrm{CH})$, 2.79 (dd, 1H, $\left.J=13.2,9.6 \mathrm{~Hz}, 1 / 2 \mathrm{CH}_{2} \mathrm{Ph}\right), 3.07$ (d, $\left.2 \mathrm{H}, J=6.3 \mathrm{~Hz}, \mathrm{C}_{2}-\mathbf{H}\right), 3.19(\mathrm{~m}, 1 \mathrm{H}$, THP(1H)), $3.31\left(\mathrm{dd}, 1 \mathrm{H}, J=13.2,2.6 \mathrm{~Hz}, 1 / 2 \mathrm{CH}_{2} \mathrm{Ph}\right), 3.48\left(\mathrm{~m}, 1 \mathrm{H}, 1 / 2 \mathrm{C}_{11},-\mathbf{H}\right), 3.54(\mathrm{~m}, 1 \mathrm{H}$, THP(1H)), $3.86\left(\mathrm{~m}, 1 \mathrm{H}, 1 / 2 \mathrm{C}_{11}-\mathbf{H}\right), 4.10\left(\mathrm{~m}, 1 \mathrm{H}, \mathrm{C}_{4}-\mathbf{H}\right), 4.21\left(\mathrm{~m}, 2 \mathrm{H}, \mathrm{NCHCH}_{2}\right), 4.57(\mathrm{~m}, 1 \mathrm{H}$, THP(1H)), 4.69 (m, 1트, NCH), 7.18-7.36 (m, 5H, ArH); ${ }^{13} \mathbf{C}-\mathbf{N M R}\left(67.5 \mathbf{M H z}, \mathbf{C D C l}_{3}\right) \delta$ 14.5, $16.7,16.8,19.4,19.6,20.3,20.7,25.5,27.1,27.2,30.6,30.8,30.9,35.2,37.9,40.4,40.5$, 40.9, 
45.6, 55.1, 62.2, 61.9, 66.3, 70.2, 70.3, 73.7, 73.9, 98.6, 99.0, 127.3, 128.9, 129.3, 135.1, 153.4, 173.1; HRMS [FAB, m-NBA] calcd for $\mathrm{C}_{30} \mathrm{H}_{47} \mathrm{O}_{6} \mathrm{NNa}$ [M+Na ${ }^{+}$: 540.3301; found: 540.3309.

Absolute configuration of C3 was determined by modified Mosher ester analysis.*

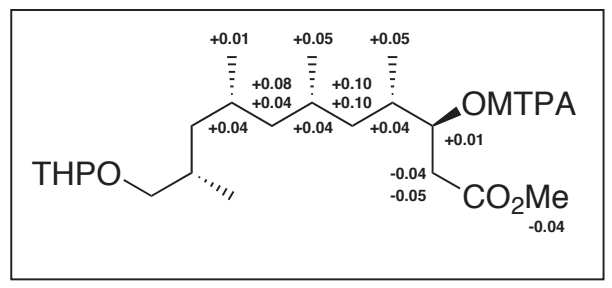

$\Delta \delta=\left(\delta_{S}-\delta_{R}\right)$ for $(R)$ - and $(S)$-MTPA derivatives

* Ohtani, I.; Kusumi, J.; Kashman, Y.; Kakisawa, H. J. Am. Chem. Soc. 1991, 113, 4092.

$(3 S, 4 S, 6 S, 8 R, 10 S)-3-($ tert-Butyldimethylsilyloxy)-4,6,8,10-tetramethyl-11-(tetrahydropyran2'-yloxy)undecanoic acid (10)

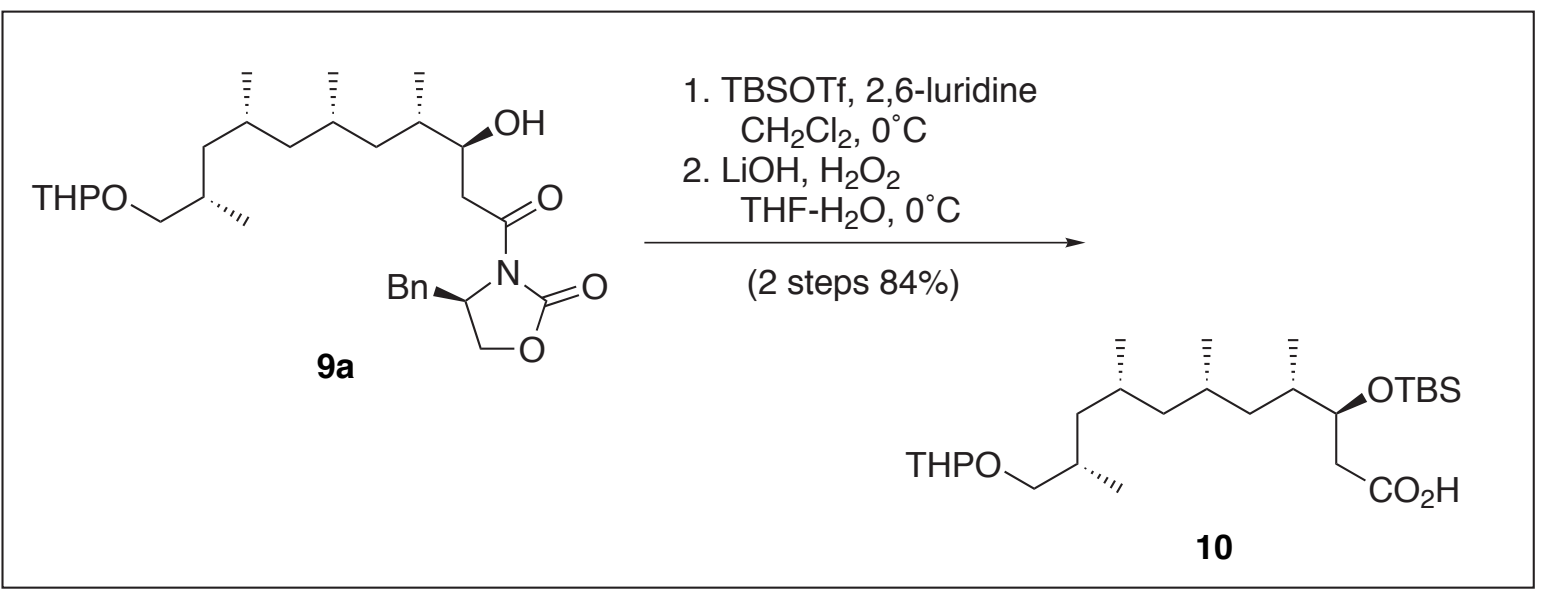

To a $0^{\circ} \mathrm{C}$ stirred solution of $9 \mathbf{a}(453.0 \mathrm{mg}, 0.876 \mathrm{mmol})$ in $\mathrm{CH}_{2} \mathrm{Cl}_{2}(9 \mathrm{ml})$ was added 2,6-lutidine (170 $\mu \mathrm{l}, 1.49 \mathrm{mmol})$ followed by TBSOTf $(260 \mu \mathrm{l}, 1.14 \mathrm{mmol})$. After $30 \mathrm{~min}$, the reaction was quenched with water and the aqueous phase was extracted with $\mathrm{CH}_{2} \mathrm{Cl}_{2}$. The combined organic extracts were dried over anhydrous $\mathrm{Na}_{2} \mathrm{SO}_{4}$ and concentrated in vacuo. This residue was employed in the next reaction without further purification. The resulting silyl ether was dissolved in THF $(6 \mathrm{ml})$ and $\mathrm{H}_{2} \mathrm{O}(2 \mathrm{ml})$ at $0^{\circ} \mathrm{C}$. Lithium hydroxide $(72.9 \mathrm{mg}, 1.74 \mathrm{mmol})$ and hydrogen peroxide ( $30 \%$ solution in $\mathrm{H}_{2} \mathrm{O}, 590 \mu \mathrm{l}, 5.21 \mathrm{mmol}$ ) were added to the solution. After $3 \mathrm{hr}$, the reaction was quenched with sat. aq. $\mathrm{Na}_{2} \mathrm{~S}_{2} \mathrm{O}_{3}$ solution, and the aqueous phase was extracted with EtOAc. The combined organic extracts were dried over anhydrous $\mathrm{Na}_{2} \mathrm{SO}_{4}$ and concentrated in 
vacuo. Flash chromatography (5:1 hexanes/EtOAc) afforded $10(347.4 \mathrm{mg}, 2$ steps 84\%) as a colorless oil.

$[\alpha]_{\mathrm{D}}{ }^{27}=-31.5^{\circ}\left(c=0.24, \mathrm{CHCl}_{3}\right)$; IR $(\mathbf{K B r}) 3444,2955,2927,1789,1456,1385,1350,1250$,

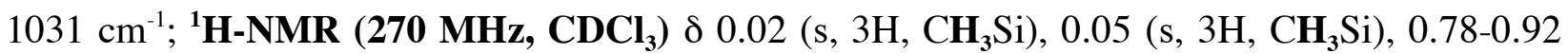
$\left(\mathrm{m}, 23 \mathrm{H}, \mathrm{CH}_{3} \times 4, \mathrm{CH}_{3} \mathrm{CSi}\right.$ and 1/2 $\left.\mathrm{CH}_{2} \times 2\right), 0.95-0.92\left(\mathrm{~m}, 3 \mathrm{H}, \mathrm{CH}_{2}\right.$ and 1/2 $\left.\mathrm{CH}_{2}\right), 1.33-1.88$ (m, $11 \mathrm{H}, 1 / 2 \mathrm{CH}_{2}, \mathbf{C H} \times 4$ and THP(6H)), $2.42\left(\mathrm{~d}, 2 \mathrm{H}, J=5.9 \mathrm{~Hz}, \mathrm{C}_{2}-\mathbf{H}\right), 3.15(\mathrm{~m}, 1 \mathrm{H}, \mathrm{THP}(1 \mathrm{H}))$, $3.50\left(\mathrm{~m}, 2 \mathrm{H}, \mathrm{THP}(1 \mathrm{H})\right.$ and $\left.1 / 2 \mathrm{C}_{11}-\mathbf{H}\right), 3.85\left(\mathrm{~m}, 1 \mathrm{H}, 1 / 2 \mathrm{C}_{11}-\mathbf{H}\right), 4.06\left(\mathrm{~m}, 1 \mathrm{H}, \mathrm{C}_{3}-\mathbf{H}\right), 4.57(\mathrm{~m}, 1 \mathrm{H}$, THP(1H)); ${ }^{13} \mathbf{C}$-NMR (67.5 MHz, $\mathbf{C D C l}_{3}$ ) $\delta$-4.7, -4.6, 15.2, 16.6, 16.7, 18.0, 19.4, 19.5, 20.6, 20.7, 20.8, 25.5, 25.8, 27.2, 27.3, 27.5, 30.6, 30.8, 30.9, 35.9, 39.3, 40.1, 40.4, 45.7, 61.9, 62.1, 72.5, 73.8, 74.0, 98.6, 99.0, 177.9; HRMS [FAB, m-NBA] calcd for $\mathrm{C}_{26} \mathrm{H}_{52} \mathrm{O}_{5} \mathrm{SiNa}\left[\mathrm{M}+\mathrm{H}^{+}\right]$: 495.3481; found: 495.3489.

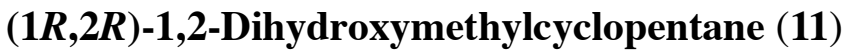

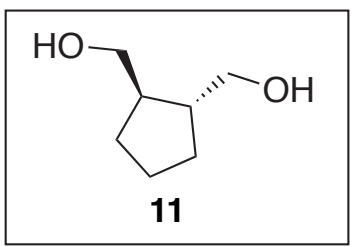

This diol was synthesized according to known protcol. [(a) Misumi, A.; Iwanaga, K.; Furuta, K.; Yamamoto, H. J. Am. Chem. Soc. 1985, 107, 3343-3345. (b) Fujimura, O.; de la Mata, F. J.; Grubbs, R. H. Organometallics 1996, 15, 1865-1871.]

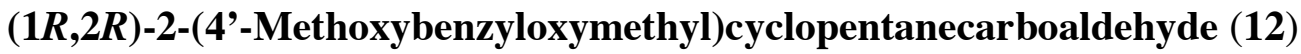

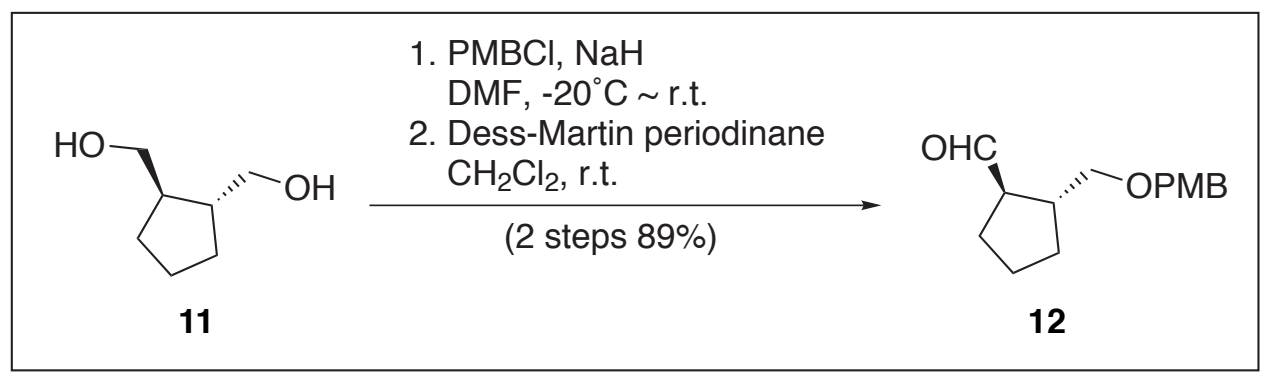

To a solution of 11 (55.0 $\mathrm{mg}, 0.42 \mathrm{mmol})$ in DMF (4.2 ml) was added sodium hydride (60\% in oil, $20 \mathrm{mg}, 0.47 \mathrm{mmol}$ ) at $-20^{\circ} \mathrm{C}$. After being stirred for $30 \mathrm{~min}$, the resulting suspension was added $\mathrm{PMBCl}(60 \mu \mathrm{L}, 0.50 \mathrm{mmol})$ and then warmed to r.t.. The reaction was quenched with 
water, and the aqueous phase was extracted with EtOAc. The combined organic extracts were dried over anhydrous $\mathrm{Na}_{2} \mathrm{SO}_{4}$ and concentrated in vacuo. Flash chromatography (7:1 hexanes/EtOAc) afforded the corresponding alcohol (111.4 mg) including unseparable impurities. To a solution of alcohol in $\mathrm{CH}_{2} \mathrm{Cl}_{2}(4.5 \mathrm{ml})$ was added Dess-Martin periodinane (283 $\mathrm{mg}, 0.67$ mmol). After being stirred at r.t. for $30 \mathrm{~min}$, the reaction mixture was quenched with sat. aq. $\mathrm{Na}_{2} \mathrm{~S}_{2} \mathrm{O}_{3}$ and sat. aq. $\mathrm{NaHCO}_{3}$, the aqueous phase was extracted with $\mathrm{CH}_{2} \mathrm{Cl}_{2}$. The combined organic extracts were dried over anhydrous $\mathrm{Na}_{2} \mathrm{SO}_{4}$ and concentrated in vacuo. Flash chromatography (20:1 hexanes/EtOAc) afforded 12 (94.5 mg, 2 steps 89\%) as a colorless oil. $[\alpha]_{\mathrm{D}}{ }^{21}=-25.9^{\circ}\left(c=0.27, \mathrm{CHCl}_{3}\right)$; IR (KBr) 2955, 2854, 1721, 1613, 1513, 1247, 1095, $1034 \mathrm{~cm}^{-}$ ${ }^{1}$; ${ }^{1} \mathbf{H}-\mathbf{N M R}\left(270 \mathrm{MHz}, \mathbf{C D C l}_{3}\right) \delta 1.31\left(\mathrm{~m}, 1 \mathrm{H}, 1 / 2 \mathrm{C}_{3}-\mathbf{H}\right), 1.57\left(\mathrm{~m}, 2 \mathrm{H}, \mathrm{C}_{4}-\mathbf{H}\right), 1.66-1.86(\mathrm{~m}, 3 \mathrm{H}$, $1 / 2 \mathrm{C}_{3}-\mathbf{H}$ and $\left.\mathrm{C}_{5}-\mathbf{H}\right), 2.33-2.51\left(\mathrm{~m}, 2 \mathrm{H}, \mathrm{C}_{1}-\mathbf{H}\right.$ and $\left.\mathrm{C}_{2}-\mathbf{H}\right), 3.25(\mathrm{dd}, 1 \mathrm{H}, J=8.9,7.3 \mathrm{~Hz}, 1 / 2$ $\mathrm{CH}_{2} \mathrm{OPMB}$ ), 3.39 (dd, 1H, $J=8.9,5.6 \mathrm{~Hz}, 1 / 2 \mathrm{CH}_{2} \mathrm{OPMB}$ ), 3.72 (s, 3H, OCH $), 4.36$ (s, 2H, $\left.\mathrm{CH}_{2} \mathrm{Ph}\right), 6.79(\mathrm{~d}, 2 \mathrm{H}, J=8.6 \mathrm{~Hz}, \mathrm{ArH}), 7.15(\mathrm{~d}, 2 \mathrm{H}, J=8.6 \mathrm{~Hz}, \mathrm{ArH}), 9.75$ (d, 1H, $J=2.0 \mathrm{~Hz}$, CHO); ${ }^{13}$ C-NMR (67.5 MHz, $\mathbf{C D C l}_{3}$ ) $\delta$ 24.9, 26.5, 29.3, 41.2, 55.2, 55.7, 72.6, 72.9, 113.7, 129.1, 130.4, 159.1, 203.7; HRMS [EI] calcd for $\mathrm{C}_{15} \mathrm{H}_{20} \mathrm{O}_{3}\left[\mathrm{M}^{+}\right]$: 248.1412; found: 248.1407.

\section{$(1 S, 1 ' R, 2 ' R)-1-[2 '-(4 '$-Methoxybenzyloxymethyl)cyclopentyl]buta-3-en-1-ol (13)}

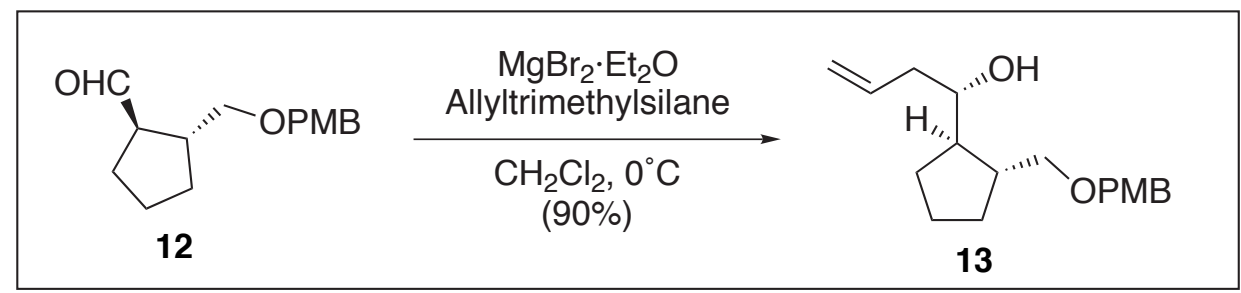

To a stirred solution of $12(111.8 \mathrm{mg}, 0.45 \mathrm{mmol})$ in $\mathrm{CH}_{2} \mathrm{Cl}_{2}(4.5 \mathrm{ml})$ was added allyltrimethylsilane $(110 \mu \mathrm{l}, 0.67 \mathrm{mmol})$ and magnesium bromide diethyl etherate $(116.4 \mathrm{mg}$, $0.45 \mathrm{mmol}$ ) at $0^{\circ} \mathrm{C}$. The reaction was stirred for $7 \mathrm{hr}$, then quenched with water. The aqueous phase was extracted with $\mathrm{CH}_{2} \mathrm{Cl}_{2}$. The combined organic extracts were dried over anhydrous $\mathrm{Na}_{2} \mathrm{SO}_{4}$ and concentrated in vacuo. Flash chromatography (25:1 hexanes/EtOAc) afforded 13 (117.3 mg, 90\%) along with its epimer (6.0 mg, 4.5\%).

$[\alpha]_{\mathrm{D}}{ }^{21}=+6.1^{\circ}\left(c=0.30, \mathrm{CHCl}_{3}\right)$; IR (KBr) 3432, 2950, 2865, 1613, 1513, 1248, 1091, $1036 \mathrm{~cm}^{-}$

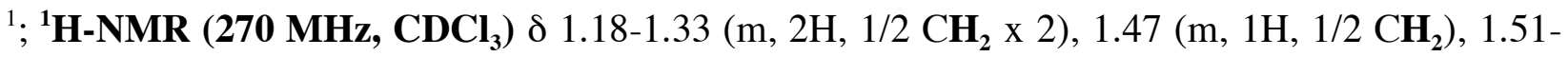
$1.63\left(\mathrm{~m}, 2 \mathrm{H}, 1 / 2 \mathrm{CH}_{2}\right.$ and $\left.\mathrm{C}_{1},-\mathbf{H}\right), 1.72-1.84\left(\mathrm{~m}, 2 \mathrm{H}, 1 / 2 \mathrm{CH}_{2} \times 2\right), 2.02-2.17\left(\mathrm{~m}, 2 \mathrm{H}, 1 / 2 \mathrm{C}_{2}-\mathbf{H}\right.$ 
and $\left.\mathrm{C}_{2},-\mathbf{H}\right), 2.39\left(\mathrm{~m}, 1 \mathrm{H}, 1 / 2 \mathrm{C}_{2}-\mathbf{H}\right), 3.20\left(\mathrm{t}, 1 \mathrm{H}, J=8.9 \mathrm{~Hz}, 1 / 2 \mathrm{CH}_{2} \mathrm{OPMB}\right), 3.41\left(\mathrm{~m}, 1 \mathrm{H}, \mathrm{C}_{1}-\mathbf{H}\right)$, $3.53\left(\mathrm{dd}, 1 \mathrm{H}, J=8.9,4.6 \mathrm{~Hz}, 1 / 2 \mathrm{CH}_{2} \mathrm{OPMB}\right), 3.81$ (s, 3H, OCH $\mathbf{H}_{3}, 4.47$ (d, 1H, $J=11.9 \mathrm{~Hz}, 1 / 2$ $\left.\mathrm{CH}_{2} \mathrm{Ph}\right), 4.53\left(\mathrm{~d}, 1 \mathrm{H}, J=11.9 \mathrm{~Hz}, 1 / 2 \mathrm{CH}_{2} \mathrm{Ph}\right), 5.10\left(\mathrm{~m}, 2 \mathrm{H}, \mathrm{C}_{4}-\mathbf{H}\right), 5.98\left(\mathrm{~m}, 1 \mathrm{H}, \mathrm{C}_{3}-\mathbf{H}\right), 6.89$ (d, $2 \mathrm{H}, J=8.6 \mathrm{~Hz}, \operatorname{ArH}), 7.26(\mathrm{~d}, 2 \mathrm{H}, J=8.6 \mathrm{~Hz}, \mathrm{ArH}) ;{ }^{13} \mathbf{C}-\mathbf{N M R}\left(\mathbf{6 7 . 5} \mathbf{M H z}, \mathbf{C D C l}_{3}\right) \delta 24.5,29.9$, 30.9, 40.4, 43.9, 51.6, 55.2, 72.8, 74.2, 74.9, 113.8, 116.4, 129.4, 129.6, 135.7, 159.2; HRMS [EI] calcd for $\mathrm{C}_{18} \mathrm{H}_{26} \mathrm{O}_{3}\left[\mathrm{M}^{+}\right]: 290.1882$; found: 290.1887 .

Stereochemical assignment of the newly created asymmetric center was achieved by Mosher's method.

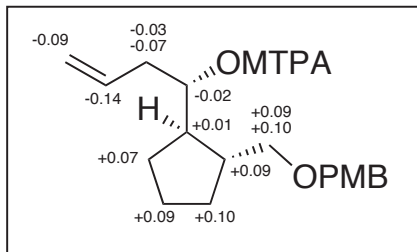

$$
\Delta \delta=\left(\delta_{S}-\delta_{R}\right) \text { for }(R) \text { - and }(S) \text {-MTPA ester of } \mathbf{1 3}
$$

(1'R,2'R,4S)-4-(tert-Butyldimethylsilyloxy)-4-[2'-(4'’-methoxybenzyloxymethyl)cyclopentyl]butene (13a)

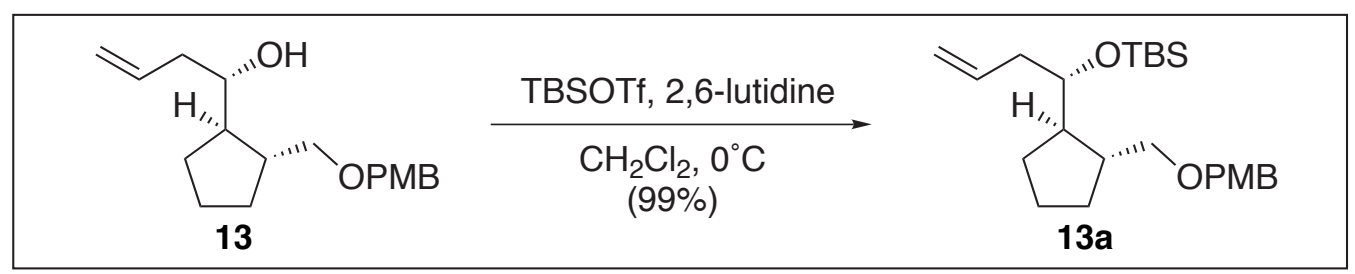

To a solution of $13(1.91 \mathrm{~g}, 6.61 \mathrm{mmol})$ in $\mathrm{CH}_{2} \mathrm{Cl}_{2}(66 \mathrm{ml})$ at $0^{\circ} \mathrm{C}$ was added 2,6-lutidine $(1.1 \mathrm{ml}$, $9.92 \mathrm{mmol})$ followed by TBSOTf $(2.0 \mathrm{ml}, 8.59 \mathrm{mmol})$. After $1 \mathrm{hr}$, the reaction was quenched with water, and the aqueous phase was extracted with $\mathrm{CH}_{2} \mathrm{Cl}_{2}$. The combined organic extracts were dried over anhydrous $\mathrm{Na}_{2} \mathrm{SO}_{4}$ and concentrated in vacuo. Flash chromatography (50:1 hexanes/EtOAc) afforded 13a (2.65 g, 99\%) as a colorless oil.

$[\alpha]_{\mathrm{D}}{ }^{22}=-8.6^{\circ}\left(c=0.27, \mathrm{CHCl}_{3}\right)$; IR (KBr) 2953, 2856, 1471, 1249, $1095 \mathrm{~cm}^{-1} ;{ }^{1} \mathbf{H}-\mathbf{N M R}(\mathbf{2 7 0}$ MHz, $\left.\mathbf{C D C l}_{3}\right) \delta 0.06\left(\mathrm{~s}, 6 \mathrm{H}, \mathrm{CH}_{3} \mathrm{Si}\right), 0.90\left(\mathrm{~s}, 9 \mathrm{H}, \mathrm{CH}_{3} \mathrm{CSi}\right), 1.23-1.36\left(\mathrm{~m}, 2 \mathrm{H}, 1 / 2 \mathrm{C}_{3},-\mathbf{H}\right.$ and $1 / 2$ $\left.\mathrm{C}_{5},-\mathbf{H}\right), 1.44-1.74\left(\mathrm{~m}, 5 \mathrm{H}, \mathrm{C}_{1},-\mathbf{H}, 1 / 2 \mathrm{C}_{3},-\mathbf{H}, \mathrm{C}_{4},-\mathbf{H}\right.$ and $\left.1 / 2 \mathrm{C}_{5},-\mathbf{H}\right), 2.15\left(\mathrm{~m}, 1 \mathrm{H}, \mathrm{C}_{2},-\mathbf{H}\right), 2.26(\mathrm{~m}$, $\left.2 \mathrm{H}, \mathrm{C}_{3}-\mathbf{H}\right), 3,18\left(\mathrm{t}, 1 \mathrm{H}, J=8.6 \mathrm{~Hz}, 1 / 2 \mathrm{CH}_{2} \mathrm{OPMB}\right), 3.46(\mathrm{dd}, 1 \mathrm{H}, J=8.6,4.9 \mathrm{~Hz}, 1 / 2$ $\left.\mathrm{CH}_{2} \mathrm{OPMB}\right), 3.64\left(\mathrm{~m}, 1 \mathrm{H}, \mathrm{C}_{4}-\mathbf{H}\right), 3.80\left(\mathrm{~s}, 3 \mathrm{H}, \mathrm{OCH}_{3}\right), 4.40\left(\mathrm{~d}, 2 \mathrm{H}, J=11.5 \mathrm{~Hz}, 1 / 2 \mathrm{CH}_{2} \mathrm{Ph}\right), 4.46$ 
$\left(\mathrm{d}, 2 \mathrm{H}, J=11.5 \mathrm{~Hz}, 1 / 2 \mathrm{CH}_{2} \mathrm{Ph}\right), 5.01\left(\mathrm{~m}, 2 \mathrm{H}, \mathrm{C}_{1}-\mathbf{H}\right), 5.85\left(\mathrm{~m}, 1 \mathrm{H}, \mathrm{C}_{2}-\mathbf{H}\right), 6.88(\mathrm{~d}, 2 \mathrm{H}, J=8.2 \mathrm{~Hz}$, ArH), 7.27 (d, 2H, $J=8.2 \mathrm{~Hz}, \operatorname{ArH}) ;{ }^{13} \mathbf{C}-\mathbf{N M R}\left(67.5\right.$ MHz, $\left.\mathbf{C D C l}_{3}\right) \delta$-4.6, -4.2, 18.0, 25.5, 25.9, 29.5, 30.5, 39.8, 41.0, 46.8, 55.2, 72.6, 74.7, 75.5, 113.7, 116.5, 129.1, 130.9, 135.2, 150.0; HRMS [FAB, m-NBA] calcd for $\mathrm{C}_{24} \mathrm{H}_{39} \mathrm{O}_{3} \mathrm{Si}\left[\mathrm{M}-\mathrm{H}^{+}\right]$: 403.2668; found: 403.2669.

\section{(1'R,2'R,3S)-3-(tert-Butyldimethylsilyloxy)-3-[2'-(4'’-methoxybenzyloxymethyl)- cyclopentyl]propanal (13b)}

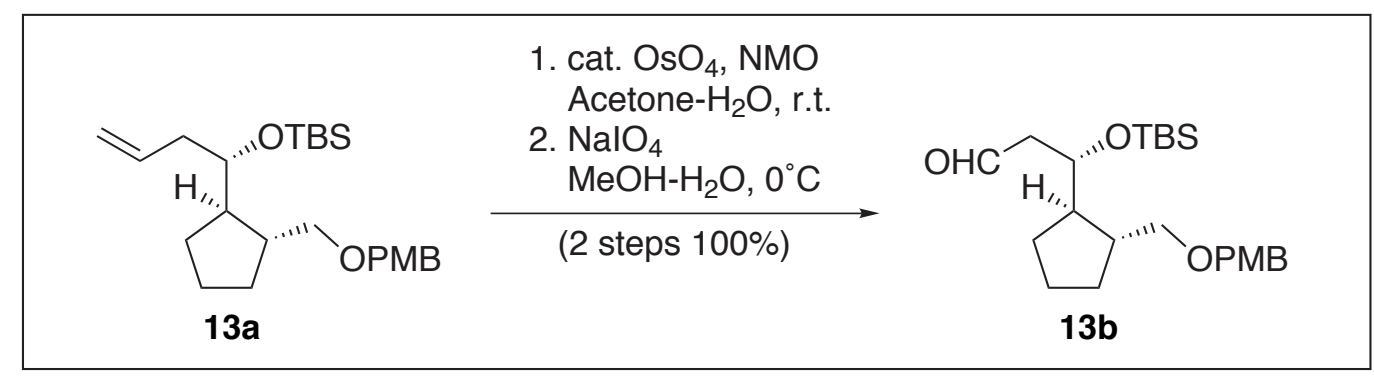

To a solution of $13 \mathrm{a}(515.8 \mathrm{mg}, 1.27 \mathrm{mmol})$ in acetone $(12 \mathrm{ml})$ and $\mathrm{H}_{2} \mathrm{O}(12 \mathrm{ml})$ were added osmium tetraoxide (32.4 $\mathrm{mg}, 0.127 \mathrm{mmol})$ and NMO (600 $\mathrm{mg}, 5.10 \mathrm{mmol})$, and the reaction was stirred at r.t. for $4 \mathrm{hr}$. The reaction mixture was quenched with sat. aq. $\mathrm{Na}_{2} \mathrm{~S}_{2} \mathrm{O}_{3}$ solution and the aqueous phase was extracted with EtOAc. The combined organic extracts were dried over anhydrous $\mathrm{Na}_{2} \mathrm{SO}_{4}$ and concentrated in vacuo to give the corresponding diol as a yellow oil.

The crude diol was dissolved in $\mathrm{MeOH}(18 \mathrm{ml})$ and $\mathrm{H}_{2} \mathrm{O}(6 \mathrm{ml})$. To the reaction mixture was added sodium metaperiodate $(545 \mathrm{mg}, 2.55 \mathrm{mmol})$ at $0^{\circ} \mathrm{C}$. The mixture was stirred for $2 \mathrm{hr}$, then quenched with sat. aq. $\mathrm{Na}_{2} \mathrm{~S}_{2} \mathrm{O}_{3}$ solution. The aqueous phase was extracted with EtOAc. The combined organic extracts were dried over anhydrous $\mathrm{Na}_{2} \mathrm{SO}_{4}$ and concentrated in vacuo. Flash chromatography (40:1 hexanes/EtOAc) afforded 13b (532.7 mg, 2 steps 100\%) as a colorless oil. $[\alpha]_{\mathrm{D}}^{22}=-14.4^{\circ}\left(c=0.36, \mathrm{CHCl}_{3}\right)$; IR (KBr) 2954, 2857, 1725, 1614, 1513, 1249, 1093, $1037 \mathrm{~cm}^{-}$

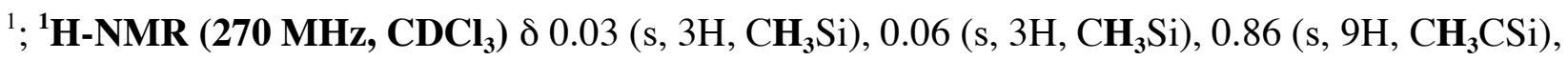
1.32-1.75 (m, 6H, $\mathrm{C}_{3},-\mathbf{H}, \mathrm{C}_{4},-\mathbf{H}$ and $\left.\mathrm{C}_{5},-\mathbf{H}\right), 1.83$ (m, 1H, $\left.\mathrm{C}_{1},-\mathbf{H}\right), 2.04$ (m, 1H, $\left.\mathrm{C}_{2},-\mathbf{H}\right), 2.48$ (ddd, $\left.1 \mathrm{H}, J=15.8,5.9,2.6 \mathrm{~Hz}, 1 / 2 \mathrm{C}_{2}-\mathbf{H}\right), 2.58\left(\mathrm{ddd}, 1 \mathrm{H}, J=15.8,4.9,1.7 \mathrm{~Hz}, 1 / 2 \mathrm{C}_{2}-\mathbf{H}\right), 3.31(\mathrm{~m}, 2 \mathrm{H}$, $\left.\mathrm{CH}_{2} \mathrm{OPMB}\right), 3.80\left(\mathrm{~s}, 3 \mathrm{H}, \mathrm{OCH}_{3}\right), 4.20\left(\mathrm{~m}, 1 \mathrm{H}, \mathrm{C}_{3}-\mathbf{H}\right), 4.41\left(\mathrm{~m}, 2 \mathrm{H}, \mathrm{CH}_{2} \mathrm{Ph}\right), 6.87$ (d, 2H, J= 8.6 $\mathrm{Hz}, \mathrm{ArH}), 7.24$ (d, 2H, ArH), 9.73 (m, 1H, CHO); ${ }^{13} \mathbf{C}-\mathbf{N M R}\left(67.5\right.$ MHz, $\mathbf{C D C l}_{3}$ ) $\delta$-4.6, 17.9, 25.2, 25.8, 28.0, 30.3 41.0, 48.6, 48.7, 55.2, 70.9, 72.8, 74.5, 113.7, 129.2, 130.6, 159.1, 202.5; HRMS [FAB, m-NBA] calcd for $\mathrm{C}_{23} \mathrm{H}_{37} \mathrm{O}_{4} \mathrm{Si}\left[\mathrm{M}-\mathrm{H}^{+}\right]$: 405.2461 ; found: 405.2451 . 
$(1 ' R, 2 ' R, 5 S)-5$-(tert-Butyldimethylsilyloxy)-5-[2'-(4''-methoxybenzyloxymethyl)cyclopentyl]penta-2E-enal (14)

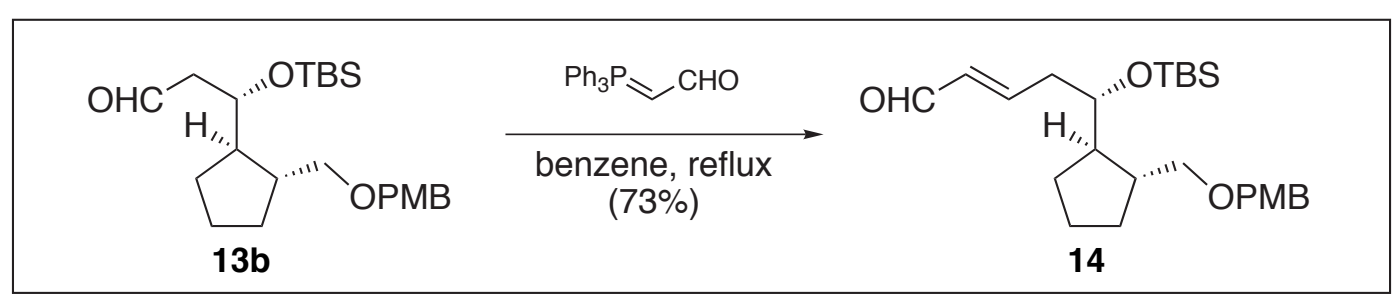

To a solution of $\mathbf{1 3 b}(20.0 \mathrm{mg}, 49.2 \mu \mathrm{mol})$ in benzene $(1.0 \mathrm{ml})$ was added triphenylphosphoranylidene acetoaldehyde $(45.0 \mathrm{mg}, 98.5 \mu \mathrm{mol})$, and the resulting solution was stirred at $80^{\circ} \mathrm{C}$ for 2 days. The solvent was then removed in vacuo and the residue was purified by flash chromatography (30:1 hexanes/EtOAc) to afford $14(15.5 \mathrm{mg}, 73 \%)$ as a yellow oil. $[\alpha]_{\mathrm{D}}{ }^{23}=+8.3^{\circ}\left(c=0.35, \mathrm{CHCl}_{3}\right)$; IR (KBr) 2953, 2857, 1694, 1513, 1249, 1091, $1038 \mathrm{~cm}^{-1} ;{ }^{1} \mathbf{H}-$ NMR (270 MHz, $\left.\mathbf{C D C l}_{3}\right) \delta 0.04$ (s, 3H, CH $\left.\mathbf{C H}_{3} \mathrm{Si}\right), 0.06$ (s, 3H, $\left.\mathrm{CH}_{3} \mathrm{Si}\right), 0.88$ (s, 9H, CH $\mathrm{CSi}_{3}$, 1.28-1.71 (m, 7H, $\mathrm{C}_{3},-\mathbf{H}, \mathrm{C}_{4}-\mathbf{H}, \mathrm{C}_{5}-\mathbf{H}$ and $\left.\mathrm{C}_{1}-\mathbf{H}\right), 2.08\left(\mathrm{~m}, 1 \mathrm{H}, \mathrm{C}_{2},-\mathbf{H}\right), 2.49\left(\mathrm{~m}, 2 \mathrm{H}, \mathrm{C}_{4}-\mathbf{H}\right), 3.22$ (t, $\left.1 \mathrm{H}, J=8.6 \mathrm{~Hz}, 1 / 2 \mathrm{CH}_{2} \mathrm{OPMB}\right), 3.39$ (dd, $\left.1 \mathrm{H}, J=8.6 \mathrm{~Hz}, 1 / 2 \mathrm{CH}_{2} \mathrm{OPMB}\right), 3.78\left(\mathrm{~m}, 1 \mathrm{H}, \mathrm{C}_{5^{-}}\right.$ H), $3.80\left(\mathrm{~s}, 3 \mathrm{H}, \mathrm{OCH}_{3}\right), 4.39\left(\mathrm{~d}, 1 \mathrm{H}, J=11.9 \mathrm{~Hz}, 1 / 2 \mathrm{CH}_{2} \mathrm{Ph}\right), 4.43(\mathrm{~d}, 1 \mathrm{H}, J=11.9 \mathrm{~Hz}, 1 / 2$ $\left.\mathrm{CH}_{2} \mathrm{Ph}\right), 6.10\left(\mathrm{dd}, 1 \mathrm{H}, J=15.5,7.9 \mathrm{~Hz}, \mathrm{C}_{2}-\mathbf{H}\right), 6.86(\mathrm{~d}, 2 \mathrm{H}, J=8.2 \mathrm{~Hz}, \mathrm{ArH}), 6.88\left(\mathrm{~m}, 1 \mathrm{H}, \mathrm{C}_{3}-\mathbf{H}\right)$, $7.24(\mathrm{~d}, 2 \mathrm{H}, J=8.2 \mathrm{~Hz}, \mathrm{ArH}), 9.47(\mathrm{~d}, 1 \mathrm{H}, J=7.9 \mathrm{~Hz}, \mathrm{CHO}) ;{ }^{13} \mathbf{C}-\mathbf{N M R}\left(\mathbf{6 7 . 5} \mathbf{~ M H z}, \mathbf{C D C l}_{3}\right) \delta$ 4.6, -4.3, 18.0, 25.2, 25.8, 29.2, 30.3, 38.4, 41.4, 47.7, 55.2, 72.7, 74.5, 74.6, 113.7, 129.2, 130.7, 134.7, 155.2, 159.1, 193.9; HRMS [EI] calcd for $\mathrm{C}_{25} \mathrm{H}_{40} \mathrm{O}_{4} \mathrm{Si}\left[\mathrm{M}^{+}\right]$: 432.2696; found: 432.2702 .

\section{$(1 ' R, 2 ' R, 7 S)-2-B r o m o-7-(t e r t-b u t y l d i m e t h y l s i l y l o x y)-7-[2 '-(4 '$ '-methoxybenzyloxy-} methyl)cyclopentyl]hepta-2E,4Z-dienenitrile (14a)

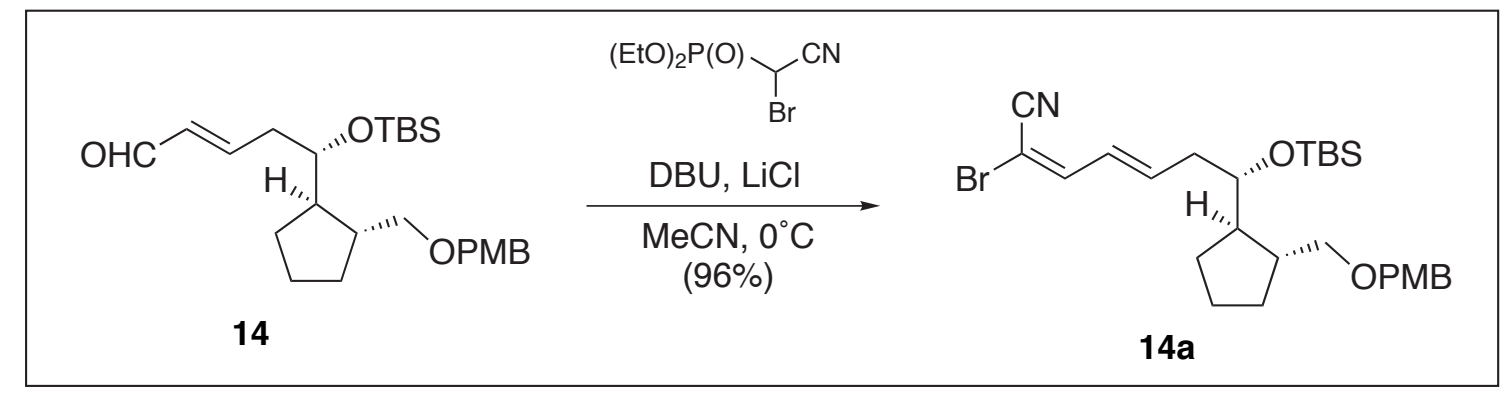

To a solution of $14(39.8 \mathrm{mg}, 92.1 \mu \mathrm{mol})$ and diethyl bromo(cyano)methylphosphate $(47.0 \mathrm{mg}$, $184 \mu \mathrm{mol})$ in $\mathrm{MeCN}(1 \mathrm{ml})$ at $0^{\circ} \mathrm{C}$ were added $\mathrm{DBU}(20 \mu \mathrm{l}, 138 \mu \mathrm{mol})$ and lithium chloride $(6.0$ 
$\mathrm{mg}, 138 \mu \mathrm{mol})$. The solution was stirred for $2 \mathrm{hr}$, then quenched with sat. aq. $\mathrm{NaHCO}_{3}$ solution. The aqueous phase was extracted with EtOAc. The combined organic extracts were dried over anhydrous $\mathrm{Na}_{2} \mathrm{SO}_{4}$ and concentrated in vacuo. Flash chromatography (60:1 hexanes/EtOAc) afforded 14a (47.3 $\mathrm{mg}, 96 \%)$ as a colorless oil.

$[\alpha]_{\mathrm{D}}{ }^{22}=+31.0^{\circ}\left(c=0.47, \mathrm{CHCl}_{3}\right)$; IR (KBr) 2952, 2856, 2217, 1614, 1513, 1248, $1078 \mathrm{~cm}^{-1} ;{ }^{1} \mathbf{H}-$ NMR (270 MHz, CDCl $\left.{ }_{3}\right) \delta 0.03\left(\mathrm{~s}, 3 \mathrm{H}, \mathrm{CH}_{3} \mathrm{Si}\right), 0.05$ (s, 3H, CH $\left.\mathrm{CH}_{3} \mathrm{Si}\right), 0.88$ (s, 9H, CH $\mathrm{CSS}$ ), 1.25-1.71 (m, 7H, C ${ }_{3},-\mathbf{H}, \mathrm{C}_{4},-\mathbf{H}, \mathrm{C}_{5},-\mathbf{H}$ and $\left.\mathrm{C}_{1},-\mathbf{H}\right), 2.07$ (m, 1H, $\left.\mathrm{C}_{2},-\mathbf{H}\right), 2.36\left(\mathrm{~m}, 2 \mathrm{H}, \mathrm{C}_{6}-\mathbf{H}\right), 3.20$ (t, $\left.1 \mathrm{H}, J=8.6 \mathrm{~Hz}, 1 / 2 \mathrm{CH}_{2} \mathrm{OPMB}\right), 3.39$ (dd, 1H, $\left.J=8.6,5.6 \mathrm{~Hz}, 1 / 2 \mathrm{CH}_{2} \mathrm{OPMB}\right), 3.69$ (m, 1H, $\left.\mathrm{C}_{7}-\mathbf{H}\right), 3.80\left(\mathrm{~s}, 3 \mathrm{H}, \mathrm{OCH}_{3}\right), 4.39\left(\mathrm{~d}, 1 \mathrm{H}, J=11.9 \mathrm{~Hz}, 1 / 2 \mathrm{CH}_{2} \mathrm{Ph}\right), 4.44(\mathrm{~d}, 1 \mathrm{H}, J=11.9 \mathrm{~Hz}, 1 / 2$ $\left.\mathrm{CH}_{2} \mathrm{Ph}\right), 6.25\left(\mathrm{~m}, 1 \mathrm{H}, \mathrm{C}_{5}-\mathbf{H}\right), 6.36\left(\mathrm{dd}, 1 \mathrm{H}, J=15.2,10.6 \mathrm{~Hz}, \mathrm{C}_{4}-\mathbf{H}\right), 6.87(\mathrm{~d}, 2 \mathrm{H}, J=8.6 \mathrm{~Hz}$, $\operatorname{ArH}), 7.10\left(\mathrm{~d}, 1 \mathrm{H}, J=10.6 \mathrm{~Hz}, \mathrm{C}_{3}-\mathbf{H}\right), 7,24(\mathrm{~d}, 2 \mathrm{H}, J=8.6 \mathrm{~Hz}, \operatorname{ArH}) ;{ }^{13} \mathbf{C}-\mathbf{N M R}(67.5 \mathrm{MHz}$, $\left.\mathbf{C D C l}_{3}\right) \delta$-4.6, -4.3, 17.9, 25.2, 25.8, 29.3, 30.3, 38.8, 41.3, 47.4, 55.1, 72.6, 74.5, 74.7, 84.1, 113.6, 114.6, 127.6, 129.1, 130.7, 143.5, 149.7, 159.0; HRMS [FAB, m-NBA] calcd for $\mathrm{C}_{27} \mathrm{H}_{40} \mathrm{O}_{3} \mathrm{NBrSiNa}\left[\mathrm{M}+\mathrm{Na}^{+}\right]$: 556.1858; found: 556.1855.

diethyl bromo(cyano)methylphosphate $\left[(\mathrm{EtO})_{2} \mathrm{P}(\mathrm{O}) \mathrm{CH}(\mathrm{Br}) \mathrm{CN}\right]$ was prepared according to known protocol. (Iorga, B.; Ricard, L.; Savignac, P. J. Chem. Soc., Perkin Trans. 1, 2000, 3311.)

\section{(1'R,2'R,7S)-2-Bromo-7-[2'-(4'’-methoxybenzyloxymethyl)-cyclopentyl]-7-hydroxyhept-}

\section{$2 Z, 4 E$-dienenitrile (15)}

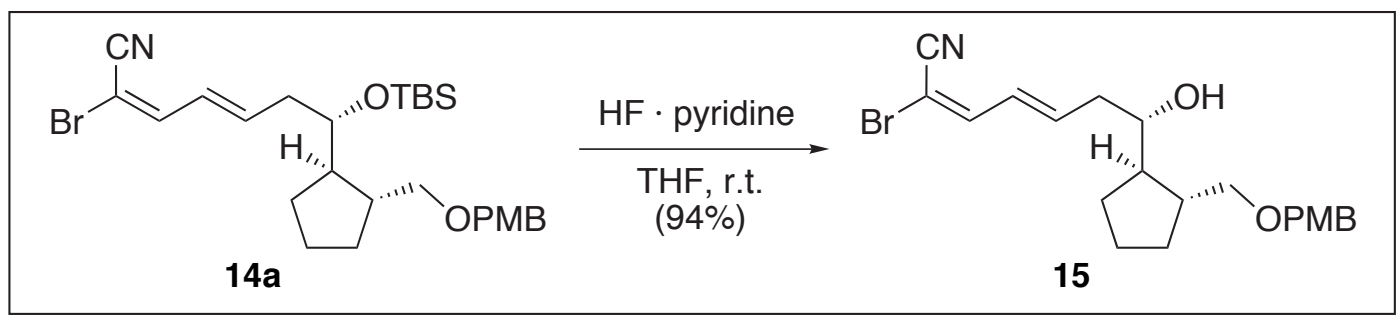

$14 \mathbf{a}(184.0 \mathrm{mg}, 345 \mu \mathrm{mol})$ was placed in a solution of HF.pyridine $(500 \mu \mathrm{l})$ in THF (500 $\mu \mathrm{l})$ and pyridine $(500 \mu \mathrm{l})$ and stirred at r.t. for 2 days. The resulting solution was filtered through a short plug of silica gel and concentrated in vacuo. The residue was purified by flash chromatography (7:1 hexanes/EtOAc) to afford $15(135.2 \mathrm{mg}, 94 \%)$.

$[\alpha]_{\mathrm{D}}{ }^{24}=+11.3^{\circ}\left(c=0.25, \mathrm{CHCl}_{3}\right)$; IR (KBr) 3406, 2949, 2222, 2216, 1613, 1513, 1248, 1075, $1035 \mathrm{~cm}^{-1}$; ${ }^{1} \mathbf{H}-\mathbf{N M R}\left(\mathbf{2 7 0} \mathbf{M H z}_{\mathbf{C D C l}}\right) \delta 1.25\left(\mathrm{~m}, 1 \mathrm{H}, 1 / 2 \mathbf{C H}_{2}\right), 1.41-1.67\left(\mathrm{~m}, 5 \mathrm{H}, 1 / 2 \mathrm{CH}_{2} \times 2\right.$, $\mathrm{C}_{1},-\mathbf{H}$ and $\left.\mathrm{C}_{4}, \mathbf{H}\right), 1.77\left(\mathrm{~m}, 1 \mathrm{H}, 1 / 2 \mathbf{C H}_{2}\right), 2.04\left(\mathrm{~m}, 1 \mathrm{H}, \mathrm{C}_{2},-\mathbf{H}\right), 2.24\left(\mathrm{~m}, 1 \mathrm{H}, 1 / 2 \mathrm{C}_{6}-\mathbf{H}\right), 2.47(\mathrm{~m}$, 
$\left.1 \mathrm{H}, 1 / 2 \mathrm{C}_{6}-\mathbf{H}\right), 3.14\left(\mathrm{dd}, 1 \mathrm{H}, J=10.6,8.6 \mathrm{~Hz}, 1 / 2 \mathrm{CH}_{2} \mathrm{OPMB}\right), 3.41$ (m, 1H, $\left.\mathrm{C}_{7}-\mathbf{H}\right), 3.56(\mathrm{dd}, 1 \mathrm{H}$, $\left.J=8.6,4.0 \mathrm{~Hz}, 1 / 2 \mathrm{CH}_{2} \mathrm{OPMB}\right), 3.81$ (s, 3H, $\left.\mathrm{OCH}_{3}\right), 4.49\left(\mathrm{~m}, 2 \mathrm{H}, \mathrm{CH}_{2} \mathrm{Ph}\right), 6.33-6.48$ (m, 2H, $\mathrm{C}_{4}-\mathbf{H}$ and $\left.\mathrm{C}_{5}-\mathbf{H}\right), 6.88(\mathrm{~d}, 2 \mathrm{H}, J=8.6 \mathrm{~Hz}, \mathrm{ArH}), 7.15\left(\mathrm{~m}, 1 \mathrm{H}, \mathrm{C}_{3}-\mathbf{H}\right), 7.24(\mathrm{~d}, 2 \mathrm{H}, J=8.6 \mathrm{~Hz}$, ArH); ${ }^{13}$ C-NMR (67.5 MHz, $\left.\mathbf{C D C l}_{3}\right) \delta$ 24.3, 29.6, 30.9, 39.6, 44.0, 52.2, 55.1, 72.8, 74.0, 74.5, 83.8, 113.7, 114.6, 127.1, 129.1, 129.4, 144.3, 150.0, 159.2; HRMS [FAB, m-NBA] calcd for $\mathrm{C}_{21} \mathrm{H}_{26} \mathrm{O}_{3} \mathrm{NBrNa}\left[\mathrm{M}+\mathrm{Na}^{+}\right]$: 442.0994; found: 442.1002 .

\section{$(1 " S, 1 " ' R, 2$ "' $R, 3 S, 4 S, 6 S, 8 R, 10 S)$-3-(tert-Butyldimethyl-silyloxy)-4,6,8,10-tetramethyl-11-} (tetrahydropyran-2'-yloxy)undecanoic acid 6"-bromo-6"-cyano-1-[2"'-(4"'methoxybenzyloxymethyl)cyclopentyl]hexa-3' $E, 5$ ' $E$-dienyl ester (15a)

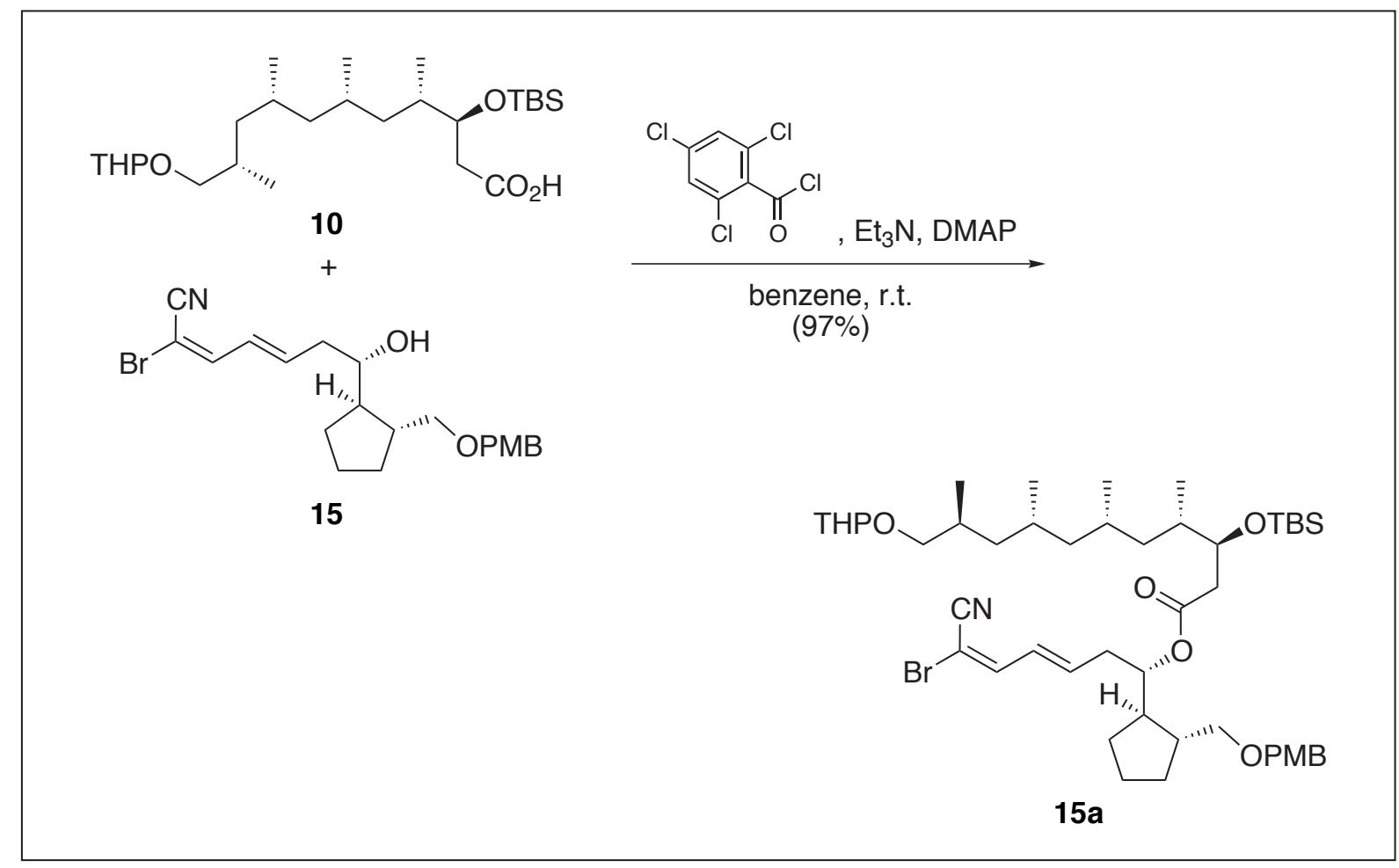

To a stirred solution of $\mathbf{1 0}(113.0 \mathrm{mg}, 0.239 \mathrm{mmol})$ in benzene $(2.4 \mathrm{ml})$ was added triethylamine (67 $\mu \mathrm{l}, 0.479 \mathrm{mmol})$ followed by 2,4,6-trichlorobenzoyl chloride (41 $\mu \mathrm{l}, 0.263 \mathrm{mmol})$ at r.t.. The resulting solution was stirred for $1 \mathrm{hr}$, then treated with a solution of $\mathbf{1 5}$ (130.4 $\mathrm{mg}, 0.311 \mathrm{mmol})$ in benzene $(1.4 \mathrm{ml})$ and DMAP $(38.0 \mathrm{mg}, 0.311 \mathrm{mmol})$ in benzene $(1.0 \mathrm{ml})$, and stirred for additional 30 min. The reaction was quenched with sat. aq. $\mathrm{NaHCO}_{3}$ solution and the aqueous phase was extracted with EtOAc. The combined organic extracts were dried over anhydrous 
$\mathrm{Na}_{2} \mathrm{SO}_{4}$ and concentrated in vacuo. Flash chromatography (25:1 hexanes/EtOAc) afforded 15a (203.5 $\mathrm{mg}, 97 \%)$ as a colorless oil.

$[\alpha]_{\mathrm{D}}{ }^{24}=-7.4^{\circ}\left(c=0.76, \mathrm{CHCl}_{3}\right)$; IR (KBr) 2957, 2927, 2856, 1732, 1259, 1092, $1034 \mathrm{~cm}^{-1} ;{ }^{1} \mathbf{H}-$

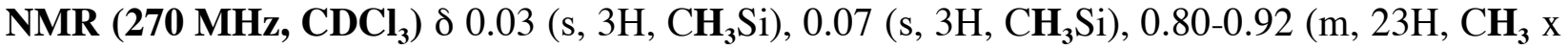
4, 1/2 $\mathrm{CH}_{2}$ x 2 and $\left.\mathrm{CH}_{3} \mathrm{CSi}\right), 0.99-1.42\left(\mathrm{~m}, 5 \mathrm{H}, 1 / 2 \mathbf{C H}_{2} \times 3\right.$ and $\left.\mathbf{C H}_{2}\right), 1.46-1.94(\mathrm{~m}, 16 \mathrm{H}$, THP(6H), $\mathbf{C H}_{2} \times 2$, CH 5 and 1/2 $\left.\mathbf{C H}_{2}\right), 2.07\left(\mathrm{~m}, 1 \mathrm{H}, \mathrm{C}_{2,},-\mathbf{H}\right), 2.31-2.60\left(\mathrm{~m}, 4 \mathrm{H}, \mathrm{C}_{2}-\mathbf{H}\right.$ and $\mathrm{C}_{2,-}$ $\mathbf{H})$, 3.10-3.39 (m, 3H, THP(1H) and $\left.\mathrm{CH}_{2} \mathrm{OPMB}\right), 3.45-3.59\left(\mathrm{~m}, 2 \mathrm{H}\right.$, THP $(1 \mathrm{H})$ and $\left.1 / 2 \mathrm{C}_{11}-\mathbf{H}\right)$, $3.80\left(\mathrm{~s}, 3 \mathrm{H}, \mathrm{OCH}_{3}\right), 3.86\left(\mathrm{~m}, 1 \mathrm{H}, 1 / 2 \mathrm{C}_{11}-\mathbf{H}\right), 4.06\left(\mathrm{~m}, 1 \mathrm{H}, \mathrm{C}_{3}-\mathbf{H}\right), 4.41(\mathrm{~d}, 1 \mathrm{H}, J=11.9 \mathrm{~Hz}, 1 / 2$ $\left.\mathrm{CH}_{2} \mathrm{Ph}\right), 4.44\left(\mathrm{~d}, 1 \mathrm{H}, J=11.9 \mathrm{~Hz}, 1 / 2 \mathrm{CH}_{2} \mathrm{Ph}\right), 4.57(\mathrm{~m}, 1 \mathrm{H}, \mathrm{THP}(1 \mathrm{H})), 4.96\left(\mathrm{~m}, 1 \mathrm{H}, \mathrm{C}_{1},-\mathbf{H}\right), 6.11$ $\left(\mathrm{m}, 1 \mathrm{H}, \mathrm{C}_{3},-\mathbf{H}\right), 6.37\left(\mathrm{dd}, 1 \mathrm{H}, J=15.2,11.2 \mathrm{~Hz}, \mathrm{C}_{4},-\mathbf{H}\right), 6.87(\mathrm{~d}, 2 \mathrm{H}, J=8.6 \mathrm{~Hz}, \mathrm{ArH}), 7.07$ (d, $\left.1 \mathrm{H}, J=11.2 \mathrm{~Hz}, \mathrm{C}_{5,-\mathbf{H}}\right), 7.24(\mathrm{~d}, 2 \mathrm{H}, J=8.6 \mathrm{~Hz}, \mathrm{ArH}) ;{ }^{13} \mathbf{C}-\mathbf{N M R}\left(67.5 \mathrm{MHz}, \mathbf{C D C l}_{3}\right) \delta-4.7,-$ $4.4,14.5,16.4,16.5,18.0,19.4,19.5,20.4,20.6,25.0,25.5,25.8,26.9,27.0,27.2,29.6,30.1$, 30.6, 30.8, 30.9, 35.4, 36.4, 39.6, 39.9, 40.8, 40.9, 41.4, 45.1, 45.7, 55.1, 61.9, 62.0, 71.5, 72.6, 73.7, 73.9, 74.0, 75.6, 85.0, 98.6, 98.9, 113.6, 114.4, 128.0, 128.9, 130.5, 141.5, 149.3, 159.0, 171.5; HRMS [FAB, m-NBA] calcd for $\mathrm{C}_{47} \mathrm{H}_{76} \mathrm{O}_{7} \mathrm{NBrSiNa}\left[\mathrm{M}+\mathrm{Na}^{+}\right]$: 896.4472; found: 896.4473.

(1'S,1'R,2'R,3S,4S,6S,8R,10S)-3-(tert-Butyldimethylsilyloxy)-11-hydroxy-4,6,8,10tetramethylundecanoic acid 6'-bromo-6'-cyano-1'-[2"'-(4"'-methoxybenzyloxymethyl)cyclopentyl]hexa-3' $E, 5^{\prime} E$-dienyl ester (15b)

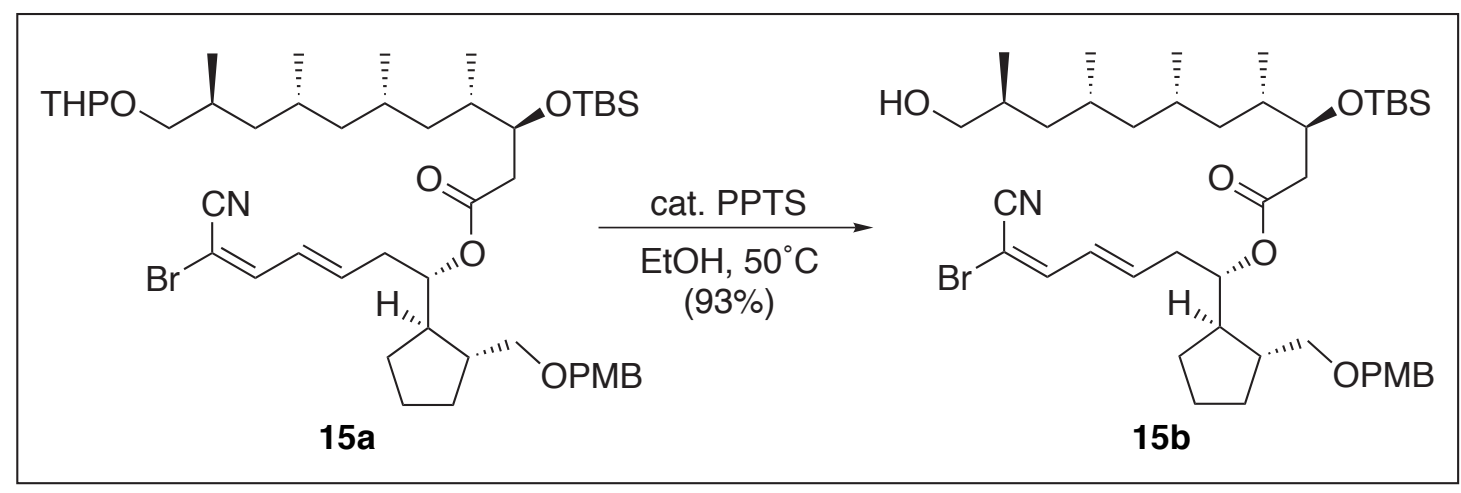

To a solution of 15a (195.0 mg, $0.223 \mathrm{mmol})$ in EtOH (5.0 ml) was added PPTS (28.0 mg, 0.112 $\mathrm{mmol}$ ), and the resulting solution was stirred at $50^{\circ} \mathrm{C}$. After $10 \mathrm{hr}$, the reaction was diluted with water, and the aqueous phase was extracted with EtOAc. The combined organic extracts were 
dried over anhydrous $\mathrm{Na}_{2} \mathrm{SO}_{4}$ and concentrated in vacuo. Flash chromatography (7:1 hexanes/EtOAc) afforded $\mathbf{1 5 b}(164.3 \mathrm{mg}, 93 \%)$ as a colorless oil.

$[\alpha]_{\mathrm{D}}{ }^{25}=-10.2^{\circ}\left(c=0.31, \mathrm{CHCl}_{3}\right)$; IR (KBr) $3500,2956,2927,1733,1250,1172,1084,1036 \mathrm{~cm}^{-}$

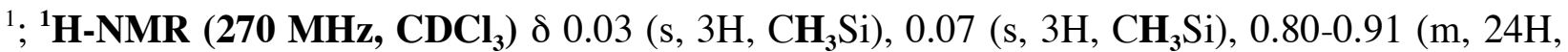
$\mathrm{CH}_{3} \times 4,1 / 2 \mathrm{CH}_{2}$ x 3 and $\mathrm{CH}_{3} \mathrm{CSi}$ ), 0.94-1.41 (m, 4H, 1/2 $\mathrm{CH}_{2}$ x 4), 1.45-1.81 (m, 9H, $\mathrm{CH}_{2}$ x 2, CH x 4 and 1/2 $\left.\mathbf{C H}_{2}\right), 1.88(\mathrm{~m}, 1 \mathrm{H}, \mathrm{CH}), 2.06(\mathrm{~m}, 1 \mathrm{H}, \mathrm{CH}), 2.31-2.60$ (m, 4H, $\mathrm{C}_{2}-\mathbf{H}$ and $\left.\mathrm{C}_{2},-\mathbf{H}\right)$, 3.24-3.47 (m, 4H, C ${ }_{11}-\mathbf{H}$ and $\left.\mathrm{CH}_{2} \mathrm{OPMB}\right), 3.80$ (s, 3H, $\left.\mathrm{OCH}_{3}\right), 4.06\left(\mathrm{~m}, 1 \mathrm{H}, \mathrm{C}_{3}-\mathbf{H}\right), 4.37$ (d, 1H, $J$ $\left.=11.9 \mathrm{~Hz}, 1 / 2 \mathrm{CH}_{2} \mathrm{Ph}\right), 4.41\left(\mathrm{~d}, 1 \mathrm{H}, J=11.9 \mathrm{~Hz}, 1 / 2 \mathrm{CH}_{2} \mathrm{Ph}\right), 4.93\left(\mathrm{~m}, 1 \mathrm{H}, \mathrm{C}_{1},-\mathbf{H}\right), 6.10(\mathrm{~m}, 1 \mathrm{H}$, $\left.\mathrm{C}_{3},-\mathbf{H}\right), 6.36\left(\mathrm{dd}, 1 \mathrm{H}, J=14.8,11.2 \mathrm{~Hz}, \mathrm{C}_{4},-\mathbf{H}\right), 6.87(\mathrm{~d}, 2 \mathrm{H}, J=8.6 \mathrm{~Hz}, \mathrm{ArH}), 7.06(\mathrm{~d}, 1 \mathrm{H}, J=$ $\left.11.2 \mathrm{~Hz}, \mathrm{C}_{5}, \mathbf{H}\right), 7.24(\mathrm{~d}, 2 \mathrm{H}, J=8.6 \mathrm{~Hz}, \mathrm{ArH}) ;{ }^{13} \mathbf{C}-\mathbf{N M R}\left(67.5 \mathbf{M H z} \mathbf{C D C l}_{3}\right) \delta$-4.7, -4.4, 14.4, 15.9, 18.0, 20.5, 20.6, 25.0, 25.8, 27.0, 27.2, 29.6, 30.1, 33.1, 35.4, 36.5, 39.5, 39.6, 41.0, 41.5, 45.1, 45.7, 55.1, 69.1, 71.7, 72.7, 73.9, 75.7, 85.0, 113.7, 114.4, 128.0, 129.0, 130.5, 141.5, 149.3, 159.0, 171.6; HRMS [FAB, m-NBA] calcd for $\mathrm{C}_{42} \mathrm{H}_{68} \mathrm{O}_{6} \mathrm{NBrSiNa}\left[\mathrm{M}+\mathrm{Na}^{+}\right]$: 812.3897; found: 812.3936 .

(1'S,1'R,2'R,3S,4S,6S,8R,10S)-3-(tert-Butyldimethylsilyloxy)-4,6,8,10-tetramethy-11oxoundecanoic acid 6'-bromo-6'-cyano-1'-[2'-(4"'-methoxybenzyloxymethyl)cyclopentyl]hexa-3' $E, 5^{\prime} Z$-dienyl ester (2)

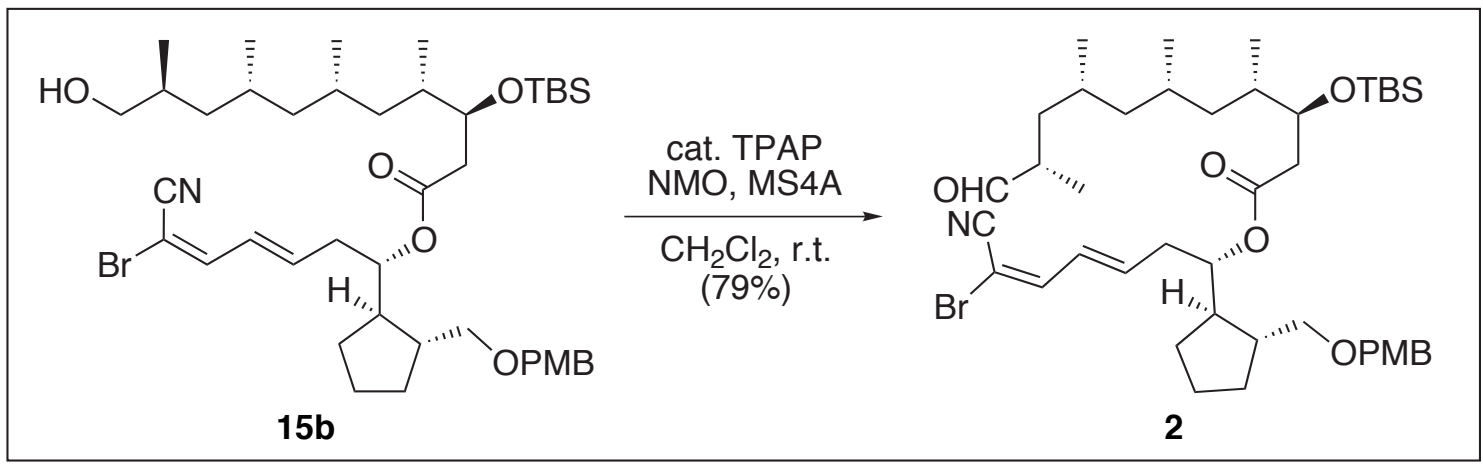

To a solution of $\mathbf{1 5 b}(153.2 \mathrm{mg}, 0.194 \mathrm{mmol})$ in $\mathrm{CH}_{2} \mathrm{Cl}_{2}(4.0 \mathrm{ml})$ were added dried MS4A $(1 \mathrm{~g})$, TPAP $(3.4 \mathrm{mg}, 9.70 \mu \mathrm{mol})$ and NMO $(45.6 \mathrm{mg}, 0.388 \mathrm{mmol})$ at r.t.. The resulting solution was stirred for $30 \mathrm{~min}$ and filtered through a silica pad. After evaporation of the filtrate, the residue was purified by flash chromatography (15:1 hexanes/EtOAc) to afford 2 (120.3 $\mathrm{mg}, 79 \%)$ as a colorless oil.

$[\alpha]_{\mathrm{D}}^{25}=-3.7^{\circ}\left(c=0.19, \mathrm{CHCl}_{3}\right)$; IR (KBr) 2955, 2929, 2856, 1731, 1513, 1462, 1249, 1084, 
$\mathrm{cm}^{-1}$; ${ }^{1} \mathrm{H}-\mathrm{NMR}\left(270 \mathrm{MHz}, \mathbf{C D C l}_{3}\right.$ ) $\delta 0.03$ (s, 3H, $\left.\mathrm{CH}_{3} \mathrm{Si}\right), 0.07$ (s, 3H, $\left.\mathrm{CH}_{3} \mathrm{Si}\right), 0.80-0.91(\mathrm{~m}$, $21 \mathrm{H}, \mathrm{CH}_{3} \times 3,1 / 2 \mathrm{CH}_{2} \times 3$ and $\left.\mathrm{CH}_{3} \mathrm{CSi}\right), 0.93-1.10\left(\mathrm{~m}, 2 \mathrm{H}, 1 / 2 \mathrm{CH}_{2} \times 2\right), 1.07(\mathrm{~d}, 3 \mathrm{H}, J=6.9 \mathrm{~Hz}$, $\left.\mathrm{C}_{10}-\mathrm{CH}_{3}\right), 1.18-1.43\left(\mathrm{~m}, 2 \mathrm{H}, 1 / 2 \mathrm{CH}_{2} \times 2\right), 1.47-1.76\left(\mathrm{~m}, 9 \mathrm{H}, \mathrm{CH}_{2} \times 2, \mathrm{CH} \times 4\right.$ and 1/2 $\left.\mathrm{CH}_{2}\right), 1.86$ (m, 1H, CH), $2.05(\mathrm{~m}, 1 \mathrm{H}, \mathbf{C H}), 2.30-2.59\left(\mathrm{~m}, 4 \mathrm{H}, \mathrm{C}_{2}-\mathbf{H}\right.$ and $\left.\mathbf{C H}_{2} \mathrm{OPMB}\right), 3.23-3.38\left(\mathrm{~m}, 2 \mathrm{H}, \mathrm{C}_{2},--\right.$ H), $3.80\left(\mathrm{~s}, 3 \mathrm{H}, \mathrm{OCH}_{3}\right), 4.05\left(\mathrm{~m}, 1 \mathrm{H}, \mathrm{C}_{3}-\mathbf{H}\right), 4.40\left(\mathrm{~d}, 1 \mathrm{H}, J=11.9 \mathrm{~Hz}, 1 / 2 \mathrm{CH}_{2} \mathrm{Ph}\right), 4.44(\mathrm{~d}, 1 \mathrm{H}, J$ $\left.=11.9 \mathrm{~Hz}, 1 / 2 \mathrm{CH}_{2} \mathrm{Ph}\right), 4.96\left(\mathrm{~m}, 1 \mathrm{H}, \mathrm{C}_{1}-\mathbf{H}\right), 6.10\left(\mathrm{~m}, 1 \mathrm{H}, \mathrm{C}_{3},-\mathbf{H}\right), 6.36(\mathrm{dd}, 1 \mathrm{H}, J=15.2,11.2 \mathrm{~Hz}$, $\left.\mathrm{C}_{4},-\mathbf{H}\right), 6.87(\mathrm{~d}, 2 \mathrm{H}, J=8.6 \mathrm{~Hz}, \operatorname{ArH}), 7.07\left(\mathrm{~d}, 1 \mathrm{H}, J=11.2 \mathrm{~Hz}, \mathrm{C}_{5},-\mathbf{H}\right), 7.24(\mathrm{~d}, 2 \mathrm{H}, J=8.6 \mathrm{~Hz}$, $\operatorname{ArH}), 9.61(\mathrm{~d}, 1 \mathrm{H}, J=1.6 \mathrm{~Hz}, \mathrm{CHO}) ;{ }^{13} \mathbf{C}-\mathbf{N M R}\left(\mathbf{6 7 . 5} \mathbf{~ M H z}, \mathbf{C D C l}_{3}\right) \delta-4.7,-4.4,13.0,14.6,18.0$, 20.4, 20.5, 25.0, 25.8, 27.3, 27.4, 29.7, 30.2, 35.4, 36.3, 36.5, 39.5, 40.7, 41.5, 44.1, 45.1,45.2, 55.2, 71.5, 72.7, 74.0, 75.6, 85.1, 113.6, 114.4, 128.1, 129.0, 130.5, 141.5, 149.3, 159.0, 171.6, 205.1; HRMS [FAB, m-NBA] calcd for $\mathrm{C}_{42} \mathrm{H}_{66} \mathrm{O}_{6} \mathrm{NBrSiNa}$ [M+Na ${ }^{+}$: 810.3740; found: 810.3770 .

$(1 ' R, 2 S, 2 ' R, 8 R, 9 S, 11 R, 13 S, 15 S, 16 S)$-16-(tert-Butyldimethyl-silyloxy)-8-hydroxy-2-[2'-(4'’methoxybenzyloxymethyl)cyclopentyl]-9,11,13,15-tetramethyl-18-oxo-oxaoctadeca-4E,6Zdiene-7-carbonitrile (19) (Intramolecular Reformatsky reaction)

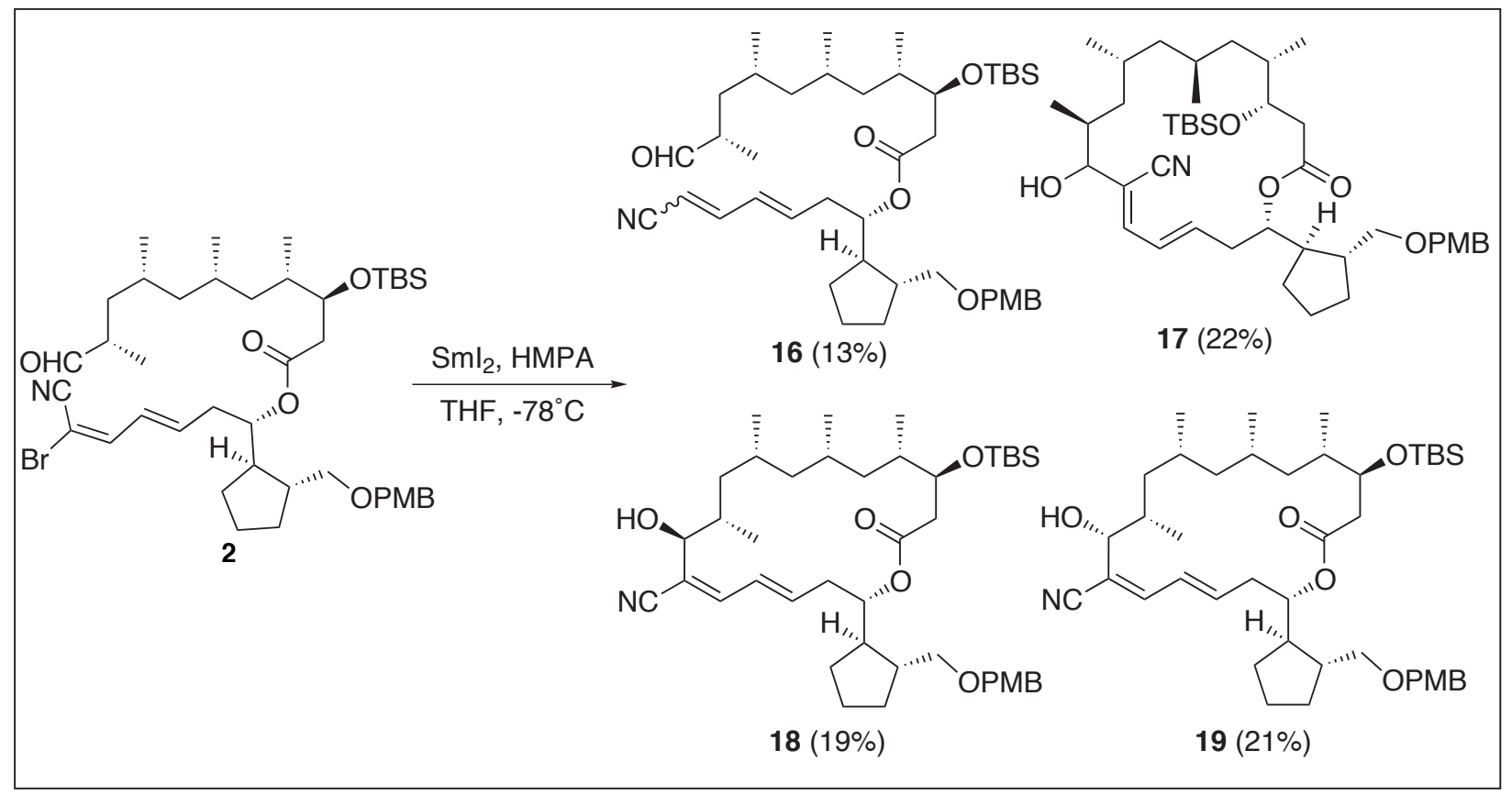

To a solution of $\mathrm{SmI}_{2}(0.1 \mathrm{M}$ solution in THF, $20 \mathrm{ml}, 2.00 \mathrm{mmol}$ ) was added HMPA ( $240 \mu \mathrm{l}, 1.37$ $\mathrm{mmol})$ at r.t. The resulting solution was cooled to $-78^{\circ} \mathrm{C}$, and $2(54.2 \mathrm{mg}, 68.9 \mu \mathrm{mol})$ was added 
dropwise over $30 \mathrm{~min}$. To the reaction was added hexane $(20 \mathrm{ml})$ and silica gel $(20 \mathrm{~g})$, and the resulting mixture was stirred at r.t. for $20 \mathrm{~min}$. The mixture was filtered through a short plug of silica gel and concentrated in vacuo. The residue was purified by flash chromatography (10:1 to 5:1 hexanes/EtOAc) to afford $\mathbf{1 6}(6.1 \mathrm{mg}, 13 \%), \mathbf{1 7}(10.5 \mathrm{mg}, 22 \%), \mathbf{1 8}(9.2 \mathrm{mg}, 19 \%)$, and desired 19 (10.2 mg, 21\%).

$19:[\alpha]_{\mathrm{D}}{ }^{24}=-19.0^{\circ}\left(c=0.54, \mathrm{CHCl}_{3}\right)$; IR (KBr) $3470,2956,2926,2853,1737,1513,1463,1249$, 1079, $1036 \mathrm{~cm}^{-1} ;{ }^{1} \mathbf{H}-\mathbf{N M R}\left(270 \mathrm{MHz}, \mathbf{C D C l}_{3}\right) \delta 0.10$ (s, 3H, $\left.\mathbf{C H}_{3} \mathrm{Si}\right), 0.11\left(\mathrm{~s}, 3 \mathrm{H}, \mathrm{CH}_{3} \mathrm{Si}\right), 0.79$ $\left(\mathrm{d}, 3 \mathrm{H}, J=6.9 \mathrm{~Hz}, \mathbf{C H}_{3}\right), 0.81\left(\mathrm{~d}, 3 \mathrm{H}, J=5.9 \mathrm{~Hz}, \mathrm{CH}_{3}\right), 0.86\left(\mathrm{~m}, 3 \mathrm{H}, \mathbf{C H}_{3}\right), 0.89$ (s, 9H, CH $\mathbf{C S i}_{3}$, $1.03\left(\mathrm{~d}, 3 \mathrm{H}, J=6.6 \mathrm{~Hz}, \mathbf{C H}_{3}\right), 0.76-1.41\left(\mathrm{~m}, 9 \mathrm{H}, \mathbf{C H}_{2} \times 4\right.$ and 1/2 $\left.\mathbf{C H}_{2}\right), 1.52-2.20(\mathrm{~m}, 7 \mathrm{H}, 1 / 2$ $\mathrm{CH}_{2}, \mathrm{CH}_{2}$ and $\left.\mathrm{CH} \times 4\right), 2.26-2.25\left(\mathrm{~m}, 6 \mathrm{H}, \mathrm{CH}_{2} \times 2\right.$ and $\left.\mathrm{CH} \times 2\right), 3.17(\mathrm{dd}, 1 \mathrm{H}, J=8.9,7.6 \mathrm{~Hz}$, 1/2 $\mathrm{CH}_{2} \mathrm{OPMB}$ ), 3,34 (dd, $1 \mathrm{H}, J=8.9,4.9 \mathrm{~Hz}, 1 / 2 \mathrm{CH}_{2} \mathrm{OPMB}$ ), 3.80 (s, 3H, OCH $\mathbf{H}_{3}, 4.04$ (m, $1 \mathrm{H}$, $\left.\mathrm{C}_{16}-\mathbf{H}\right), 4.08\left(\mathrm{~d}, 1 \mathrm{H}, J=9.9 \mathrm{~Hz}, \mathrm{C}_{8}-\mathbf{H}\right), 4.38\left(\mathrm{~d}, 1 \mathrm{H}, J=11.9 \mathrm{~Hz}, 1 / 2 \mathrm{CH}_{2} \mathrm{Ph}\right), 4.43(\mathrm{~d}, 1 \mathrm{H}, J=$ $\left.11.9 \mathrm{~Hz}, 1 / 2 \mathrm{CH}_{2} \mathrm{Ph}\right), 5.02\left(\mathrm{~m}, 1 \mathrm{H}, \mathrm{C}_{2}-\mathbf{H}\right), 6.17\left(\mathrm{~m}, 1 \mathrm{H}, \mathrm{C}_{4}-\mathbf{H}\right), 6.30(\mathrm{dd}, 1 \mathrm{H}, J=14.8,10.9 \mathrm{~Hz}$, $\left.\mathrm{C}_{5}-\mathbf{H}\right), 6.82\left(\mathrm{~d}, 1 \mathrm{H}, J=10.9 \mathrm{~Hz}, \mathrm{C}_{6}-\mathbf{H}\right), 6.87(\mathrm{~d}, 2 \mathrm{H}, J=8.6 \mathrm{~Hz}, \mathrm{ArH}), 7.24(\mathrm{~d}, 2 \mathrm{H}, J=8.6 \mathrm{~Hz}$, ArH); ${ }^{13} \mathbf{C}-N M R\left(67.5 ~ M H z, ~ \mathbf{C D C l}_{3}\right.$ ) $\delta$-5.4, -4.4, 14.9, 17.9, 18.6, 19.8, 20.7, 25.0, 25.9, 26.1, $26.2,30.0,30.3,35.3,35.6,36.2,36.4,37.6,42.7,43.4,43.6,48.1,55.3,72.6,72.9,73.8,74.0$, 75.2, 77.2, 113.8, 115.5, 126.9, 128.9, 130.7, 139.7, 144.2, 159.1, 171.5; HRMS [FAB, m-NBA] calcd for $\mathrm{C}_{42} \mathrm{H}_{67} \mathrm{O}_{6} \mathrm{NSiNa}\left[\mathrm{M}+\mathrm{Na}^{+}\right]$: 732.4635; found: 732.4639 .

$18:[\alpha]_{\mathrm{D}}^{24}=-17.6^{\circ}\left(c=1.05, \mathrm{CHCl}_{3}\right)$; IR $(\mathbf{K B r}) 3454,2955,2926,2854,1737,1513,1463,1248$, 1175, $1078 \mathrm{~cm}^{-1} ;{ }^{1} \mathbf{H}-\mathbf{N M R}\left(270 \mathrm{MHz}, \mathbf{C D C l}_{3}\right) \delta 0.99$ (s, 3H, $\left.\mathbf{C H}_{3} \mathrm{Si}\right), 0.11\left(\mathrm{~s}, 3 \mathrm{H}, \mathrm{CH}_{3} \mathrm{Si}\right), 0.77$ $\left(\mathrm{d}, 3 \mathrm{H}, J=6.9 \mathrm{~Hz}, \mathrm{CH}_{3}\right), 0.78\left(\mathrm{~d}, 3 \mathrm{H}, J=5.9 \mathrm{~Hz}, \mathrm{CH}_{3}\right), 0.86\left(\mathrm{~s}, 9 \mathrm{H}, \mathrm{CH}_{3} \mathrm{CSi}\right), 0.88\left(\mathrm{~m}, 3 \mathrm{H}, \mathrm{CH}_{3}\right)$, $1.05\left(\mathrm{~d}, 3 \mathrm{H}, J=6.9 \mathrm{~Hz}, \mathrm{CH}_{3}\right), 0.72-1.17\left(\mathrm{~m}, 5 \mathrm{H}, \mathbf{C H}_{2} \times 2\right.$ and $\left.1 / 2 \mathbf{C H}_{2}\right), 1.21-2.12\left(\mathrm{~m}, 13 \mathrm{H}, \mathbf{C H}_{2}\right.$ x 3, 1/2 $\mathrm{CH}_{2}$ and $\mathbf{C H} \times$ 6), 2.18-2.40 (m, $4 \mathrm{H}, \mathrm{C}_{3}-\mathbf{H}$ and $\left.\mathrm{C}_{17}-\mathbf{H}\right), 3.27(\mathrm{dd}, 1 \mathrm{H}, J=8.9,7.3 \mathrm{~Hz}, 1 / 2$ $\mathrm{CH}_{2} \mathrm{OPMB}$ ), 3.36 (dd, $1 \mathrm{H}, J=8.9,5.9 \mathrm{~Hz}, 1 / 2 \mathrm{CH}_{2} \mathrm{OPMB}$ ), 3.80 (s, 3H, OCH $\left.\mathbf{H}_{3}\right), 4.00(\mathrm{~m}, 1 \mathrm{H}$, $\left.\mathrm{C}_{16}-\mathbf{H}\right), 4.40\left(\mathrm{~d}, 1 \mathrm{H}, J=11.9 \mathrm{~Hz}, 1 / 2 \mathrm{CH}_{2} \mathrm{Ph}\right), 4.44\left(\mathrm{~d}, 1 \mathrm{H}, J=11.9 \mathrm{~Hz}, 1 / 2 \mathrm{CH}_{2} \mathrm{Ph}\right), 4.54(\mathrm{~d}, 1 \mathrm{H}$, $\left.J=3.3 \mathrm{~Hz}, \mathrm{C}_{8}-\mathbf{H}\right), 5.04\left(\mathrm{~m}, 1 \mathrm{H}, \mathrm{C}_{2}-\mathbf{H}\right), 5.90\left(\mathrm{~m}, 1 \mathrm{H}, \mathrm{C}_{4}-\mathbf{H}\right), 6.38\left(\mathrm{dd}, 1 \mathrm{H}, J=14.2,11.5 \mathrm{~Hz}, \mathrm{C}_{5^{-}}\right.$ H), $6.74\left(\mathrm{~d}, 1 \mathrm{H}, J=11.5 \mathrm{~Hz}, \mathrm{C}_{6}-\mathbf{H}\right), 6.87(\mathrm{~d}, 2 \mathrm{H}, J=8.6 \mathrm{~Hz}, \mathrm{ArH}), 7.25(\mathrm{~d}, 2 \mathrm{H}, J=8.6 \mathrm{~Hz}, \operatorname{ArH})$; ${ }^{13}$ C-NMR (67.5 MHz, $\mathbf{C D C l}_{3}$ ) $\delta$-5.2, -4.3, 12.4, 17.9, 18.5, 19.9, 20.7, 25.0, 25.7, 25.9, 26.0, $29.4,30.2$, 35.7, 35.9, 37.0, 37.4, 37.5, 41.6, 43.8, 46.4, 48.4, 55.2, 72.0, 72.8, 73.2, 74.3, 74.7, 
77.2, 112.4, 113.8, 126.7, 129.0, 130.7, 142.2, 145.8, 159.1, 171.3; HRMS [FAB, m-NBA] calcd for $\mathrm{C}_{42} \mathrm{H}_{67} \mathrm{O}_{6} \mathrm{NSiNa}\left[\mathrm{M}+\mathrm{Na}^{+}\right]$: 732.4635; found: 732.4642 .

NOE experiments were employed to verify the stereochemical assignment for the olefin isomers.

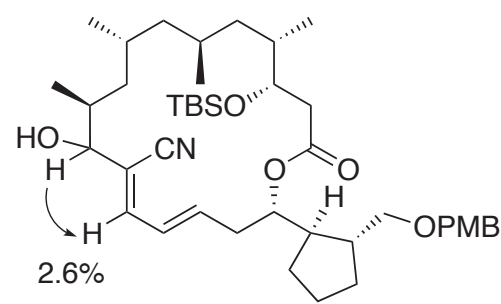

17

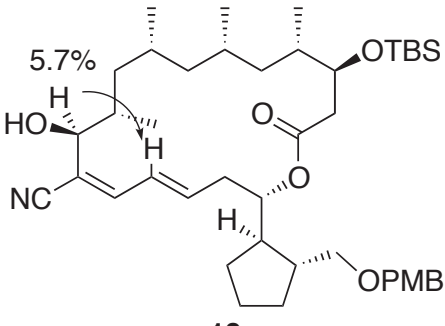

18

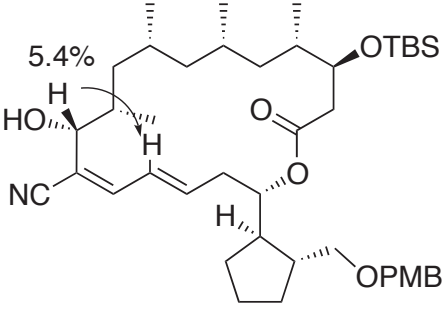

19

NOE experiments of 17, 18 and 19

$(1 ' R, 2 S, 2$ ' $R, 8 R, 9 S, 11 R, 13 S, 15 S, 16 S)$-16-(tert-Butyldimethyl-silyloxy)-8-hydroxy-2-[2'-(4'’methoxybenzyloxymethyl)cyclopentyl]-9,11,13,15-tetramethyl-18-oxo-oxaoctadeca-4E,6Zdiene-7-carbonitrile (19) (Oxidation/Reduction)

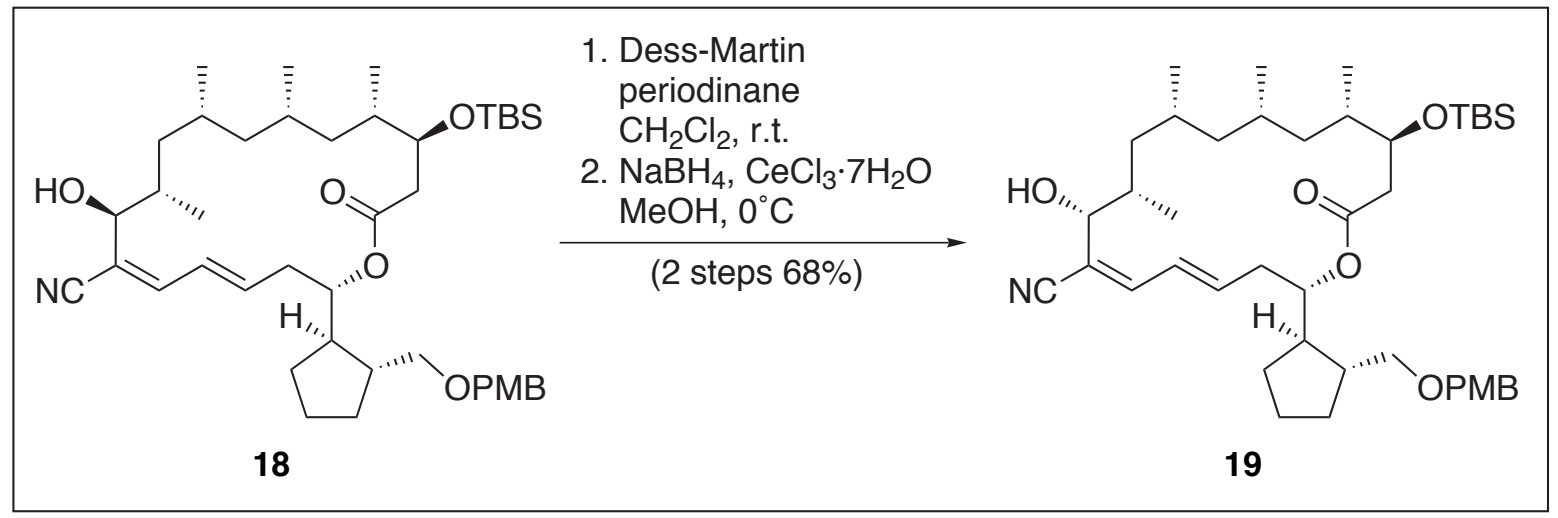

To a solution of $\mathbf{1 8}(13.2 \mathrm{mg}, 18.6 \mu \mathrm{mol})$ in $\mathrm{CH}_{2} \mathrm{Cl}_{2}(1 \mathrm{ml})$ was added Dess-Martin periodinane (22.6 mg, $55.9 \mu \mathrm{mol}$ ). After stirred for $30 \mathrm{~min}$, the reaction was quenched by sat. aq. $\mathrm{Na}_{2} \mathrm{~S}_{2} \mathrm{O}_{3}$ and sat. aq. $\mathrm{NaHCO}_{3}$ solutions, and the aqueous phase was extracted with $\mathrm{CH}_{2} \mathrm{Cl}_{2}$. The combined organic extracts were dried over anhydrous $\mathrm{Na}_{2} \mathrm{SO}_{4}$ and concentrated in vacuo. The residue was subjected to the next reaction.

To a solution of the crude ketone in $\mathrm{MeOH}(1 \mathrm{ml})$ at $0^{\circ} \mathrm{C}$ was added cerium chloride $(14.0 \mathrm{mg}$, $37.2 \mu \mathrm{mol})$ followed by sodium borohydride $(1.4 \mathrm{mg}, 37.2 \mu \mathrm{mol})$. The reaction was stirred for 10 min, and then poured into water. The aqueous phase was extracted with EtOAc. The combined organic extracts were dried over anhydrous $\mathrm{Na}_{2} \mathrm{SO}_{4}$ and concentrated in vacuo. Flash 
chromatography (8:1 hexanes/EtOAc) afforded 19 (8.5 mg, 2 steps 66\%) and 18 (0.7 mg, 2 steps $5.3 \%$ ) as a yellow oil.

$(1 ' R, 2 S, 2 ' R, 8 R, 9 S, 11 R, 13 S, 15 S, 16 S)-9,16-B i s(t e r t$-butyldimethylsilyloxy)-2-[2'-(4'’methoxybenzyloxymethyl)cyclopentyl]-9,11,13,15-tetramethyl-18-oxo-oxaoctadeca-4E,6Zdiene-7-carbonitrile (19a)

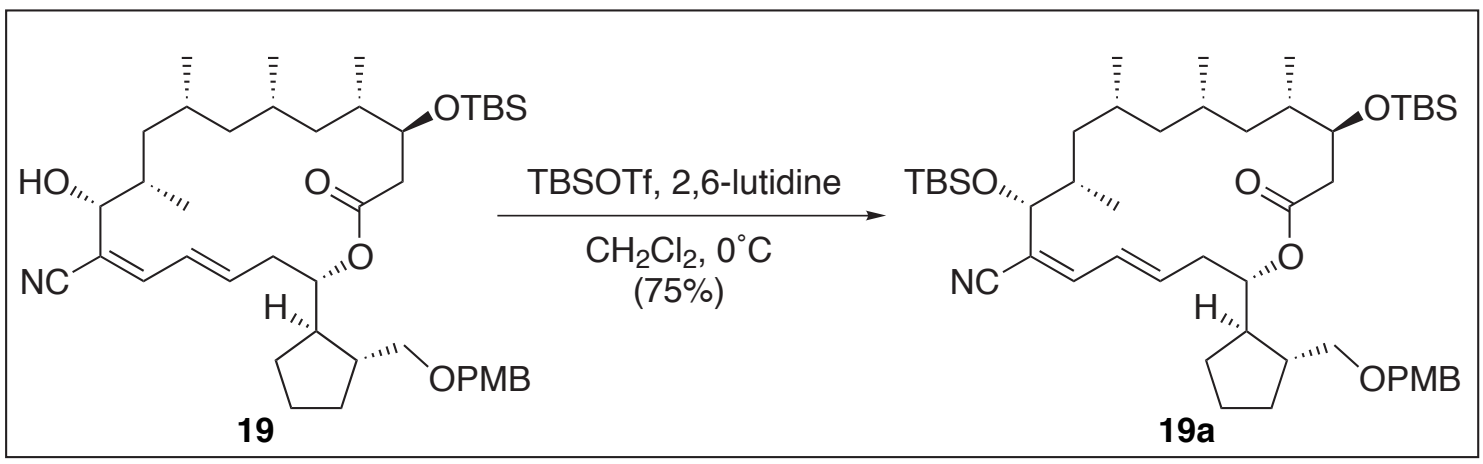

To a $0^{\circ} \mathrm{C}$ cooled solution of $\mathbf{1 9}(13.1 \mathrm{mg}, 18.4 \mu \mathrm{mol})$ in $\mathrm{CH}_{2} \mathrm{Cl}_{2}(200 \mu \mathrm{l})$ was added 2,6-lutidine (4 $\mu \mathrm{l}, 31.4 \mu \mathrm{mol})$ followed by TBSOTf $(6 \mu \mathrm{l}, 24.0 \mu \mathrm{mol})$. The resulting solution was then stirred at $0^{\circ} \mathrm{C}$ for $1 \mathrm{hr}$. The reaction was poured into water and the aqueous phase was extracted with $\mathrm{CH}_{2} \mathrm{Cl}_{2}$. The combined organic extracts were dried over anhydrous $\mathrm{Na}_{2} \mathrm{SO}_{4}$ and concentrated in vacuo. Flash chromatography (25:1 hexanes/EtOAc) afforded 19a (11.4 mg, 75\%) as a colorless oil.

$[\alpha]_{\mathrm{D}}^{25}=-37.8^{\circ}\left(c=0.69, \mathrm{CHCl}_{3}\right)$; IR (KBr) 2956, 2928, 2856, 1738, 1612, 1513, 1250, $1084 \mathrm{~cm}^{-}$

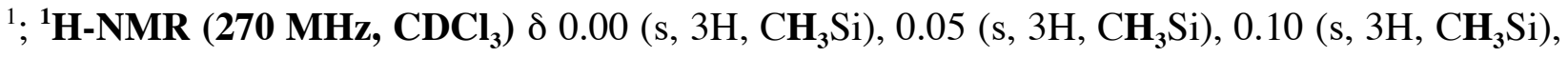

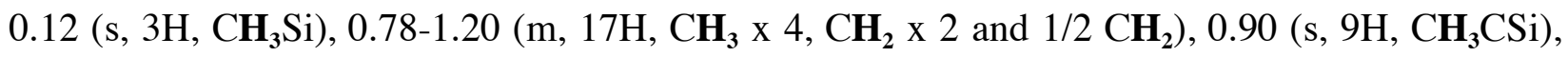
0.91 (s, 9H, $\left.\mathbf{C H}_{3} \mathrm{CSi}\right), 1.23-2.09\left(\mathrm{~m}, 11 \mathrm{H}, \mathrm{CH}_{2} \times 3,1 / 2 \mathbf{C H}_{2}\right.$ and $\left.\mathbf{C H} \times 4\right), 2.16-2.33\left(\mathrm{~m}, 5 \mathrm{H}, \mathrm{C}_{17^{-}}\right.$ $\mathbf{H}, 1 / 2 \mathrm{C}_{3}-\mathbf{H}$ and $\left.\mathbf{C H} \times 2\right), 2.52\left(\mathrm{~m}, 1 \mathrm{H}, 1 / 2 \mathrm{C}_{3}-\mathbf{H}\right), 3.17\left(\mathrm{dd}, 1 \mathrm{H}, J=8.6,8.3 \mathrm{~Hz}, 1 / 2 \mathrm{CH}_{2} \mathrm{OPMB}\right)$, $3.35\left(\mathrm{dd}, 1 \mathrm{H}, J=8.9,4.9 \mathrm{~Hz}, 1 / 2 \mathrm{CH}_{2} \mathrm{OPMB}\right), 3.80\left(\mathrm{~s}, 3 \mathrm{H}, \mathrm{OCH}_{3}\right), 3.98-4.10\left(\mathrm{~m}, 2 \mathrm{H}, \mathrm{C}_{8}-\mathbf{H}\right.$ and $\left.\mathrm{C}_{16}-\mathbf{H}\right), 4.39\left(\mathrm{~d}, 1 \mathrm{H}, J=11.9 \mathrm{~Hz}, 1 / 2 \mathrm{CH}_{2} \mathrm{Ph}\right), 4.43\left(\mathrm{~d}, 1 \mathrm{H}, J=11.9 \mathrm{~Hz}, 1 / 2 \mathrm{CH}_{2} \mathrm{Ph}\right), 5.02(\mathrm{~m}, 1 \mathrm{H}$, $\left.\mathrm{C}_{2}-\mathbf{H}\right), 6.09-6.32\left(\mathrm{~m}, 2 \mathrm{H}, \mathrm{C}_{4}-\mathbf{H}\right.$ and $\left.\mathrm{C}_{5}-\mathbf{H}\right), 6.75\left(\mathrm{~d}, 1 \mathrm{H}, J=10.6 \mathrm{~Hz}, \mathrm{C}_{6}-\mathbf{H}\right), 6.87(\mathrm{~d}, 2 \mathrm{H}, J=8.6$ $\mathrm{Hz}, \mathrm{ArH}), 7.23$ (d, 2H, $J=8.6 \mathrm{~Hz}, \mathrm{ArH}) ;{ }^{13} \mathbf{C}-\mathbf{N M R}\left(67.5 \mathbf{M H z}, \mathbf{C D C l}_{3}\right.$ ) $\delta$-5.6, -4.9, -4.6, -3.5, $15.3,17.9,18.1,18.5,20.0,20.7,22.6,25.0,25.6,25.7,26.1,26.2$, 29.7, 30.0, 30.2 , 35.6, 35.9, 36.3, 37.1, 42.9, 43.5, 48.1, 55.2, 72.6, 73.3, 73.9, 74.0, 75.3, 77.2, 113.8, 118.8, 127.1, 129.0, 130.7, 139.0, 142.9, 159.1, 171.5; HRMS [FAB, m-NBA] calcd for $\mathrm{C}_{48} \mathrm{H}_{81} \mathrm{O}_{6} \mathrm{NSi}_{2} \mathrm{Na}\left[\mathrm{M}+\mathrm{Na}^{+}\right.$]: 
846.5500; found: 846.5494 .

(1'R,2S,2'R,8R,9S,11R,13S,15S,16S)-9,16-Bis(tert-butyldimethyl-silyloxy)-2-(2'hydorxymethylcyclopentyl)-9,11,13,15-tetramethyl-18-oxo-oxaoctadeca-4E,6Z-diene-7carbonitrile (19b)

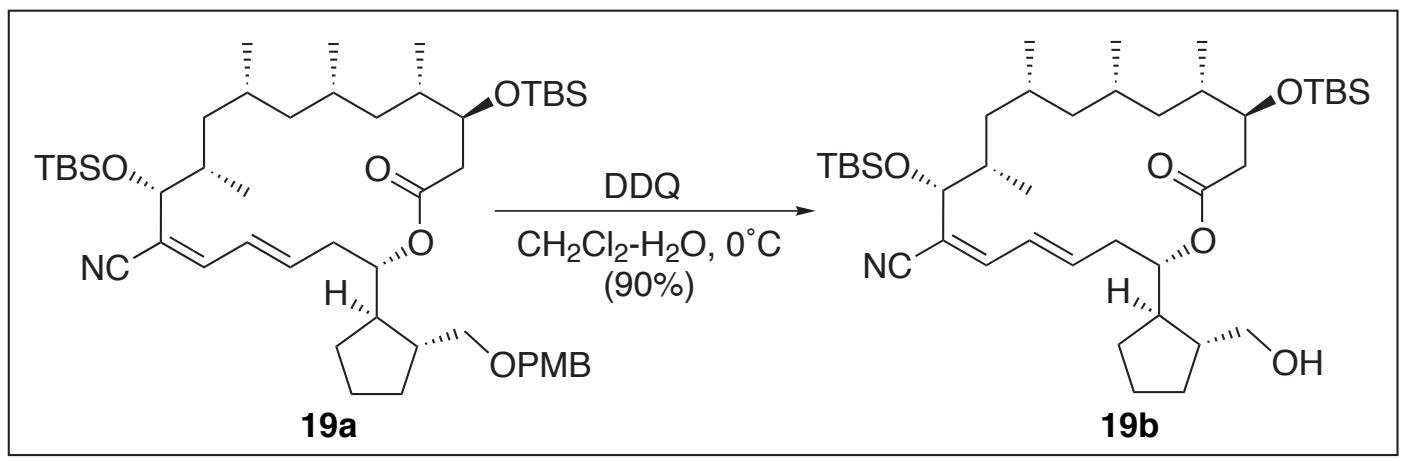

To a solution of 19a $(11.4 \mathrm{mg}, 13.9 \mu \mathrm{mol})$ in $\mathrm{CH}_{2} \mathrm{Cl}_{2}(250 \mu \mathrm{l})$ and $\mathrm{H}_{2} \mathrm{O}(50 \mu \mathrm{l})$ was added DDQ $(3.7 \mathrm{mg}, 16.6 \mu \mathrm{mol})$ at $0^{\circ} \mathrm{C}$. The reaction mixture was stirred for $15 \mathrm{~min}$, and then poured into water. The aqueous phase was extracted with EtOAc. The combined organic extracts were dried over anhydrous $\mathrm{Na}_{2} \mathrm{SO}_{4}$ and concentrated in vacuo. Flash chromatography (8:1 hexanes/EtOAc) afforded $\mathbf{1 9 b}(8.8 \mathrm{mg}, 90 \%)$ as a colorless oil.

$[\alpha]_{\mathrm{D}}^{25}=-37.2^{\circ}\left(c=0.36, \mathrm{CHCl}_{3}\right)$; IR (KBr) 3447, 2956, 2927, 2856, 1740, 1464, 1386, 1292, 1253, $1081 \mathrm{~cm}^{-1}$; ${ }^{1} \mathbf{H}-\mathbf{N M R}\left(270 \mathbf{~ M H z} \mathbf{C D C l}_{3}\right) \delta-0.01\left(\mathrm{~s}, 3 \mathrm{H}, \mathrm{CH}_{3} \mathrm{Si}\right), 0.05$ (s, 3H, CH $\mathrm{CH}_{3} \mathrm{Si}, 0.11$ $\left(\mathrm{s}, 3 \mathrm{H}, \mathrm{CH}_{3} \mathrm{Si}\right), 0.13$ (s, 3H, CH $\left.\mathbf{H}_{3} \mathrm{Si}\right), 0.80$ (d, 3H, J = 5.9 Hz, $\left.\mathrm{CH}_{3}\right), 0.81\left(\mathrm{~d}, 3 \mathrm{H}, J=7.3 \mathrm{~Hz}, \mathrm{CH}_{3}\right)$, 0.85 (d, 3H, $J=6.6 \mathrm{~Hz}, \mathrm{CH}_{3}$ ), 0.90 (s, 9H, $\mathrm{CH}_{3} \mathrm{CSi}$ ), 0.91 (s, 9H, CH $\mathbf{H}_{3} \mathrm{CSi}$ ), 0.94 (d, 3H, J = 6.3 $\mathrm{Hz}, \mathbf{C H}_{3}$ ), 0.67-1.18 (m, 5H, $\mathbf{C H}_{2} \times 2$ and 1/2 $\left.\mathbf{C H}_{2}\right), 1.23-2.09$ (m, 13H, $\mathbf{C H}_{2} \times 3,1 / 2 \mathrm{CH}_{2}$ and CH x 6), 2.21-2.39 (m, 3H, C $17-\mathbf{H}$ and 1/2 $\left.\mathrm{C}_{3}-\mathbf{H}\right), 2.54$ (m, 1H, 1/2 $\left.\mathrm{C}_{3}-\mathbf{H}\right), 3.42$ (dd, 1H, J = 10.6, $\left.6.6 \mathrm{~Hz}, 1 / 2 \mathrm{CH}_{2} \mathrm{OH}\right), 3.51\left(\mathrm{dd}, 1 \mathrm{H}, J=10.6,5.9 \mathrm{~Hz}, 1 / 2 \mathrm{CH}_{2} \mathrm{OH}\right), 3.99$ (d, $\left.1 \mathrm{H}, J=9.9 \mathrm{~Hz}, \mathrm{C}_{8}-\mathbf{H}\right)$, $4.08\left(\mathrm{~m}, 1 \mathrm{H}, \mathrm{C}_{16}-\mathbf{H}\right), 5.02\left(\mathrm{~m}, 1 \mathrm{H}, \mathrm{C}_{2}-\mathbf{H}\right), 6.15\left(\mathrm{~m}, 1 \mathrm{H}, \mathrm{C}_{4}-\mathbf{H}\right), 6.28(\mathrm{dd}, 1 \mathrm{H}, J=15.2,10.6 \mathrm{~Hz}$, $\left.\mathrm{C}_{5}-\mathbf{H}\right), 6.75\left(\mathrm{~d}, 1 \mathrm{H}, J=10.6 \mathrm{~Hz}, \mathrm{C}_{6}-\mathbf{H}\right){ }^{13} \mathbf{C}-\mathbf{N M R}\left(\mathbf{6 7 . 5} \mathbf{M H z}, \mathbf{C D C l}_{3}\right) \delta-5.6,-4.9,-4.6,-3.4$, $15.3,17.9,18.1,18.4,20.0,20.7,22.7,25.1,25.7,26.1,26.2,29.5,29.7,30.5,35.6,35.9,36.2$, 37.1, 43.4, 43.5, 45.5, 48.0, 66.7, 73.3, 73.9, 75.4, 77.2, 118.7, 127.2, 138.6, 142.8, 171.4; HRMS [FAB, m-NBA] calcd for $\mathrm{C}_{40} \mathrm{H}_{73} \mathrm{O}_{5} \mathrm{NSi}_{2} \mathrm{Na}$ [M+Na ${ }^{+}$: 726.4925 ; found: 726.4924 . 


\section{Borrelidin (1)}

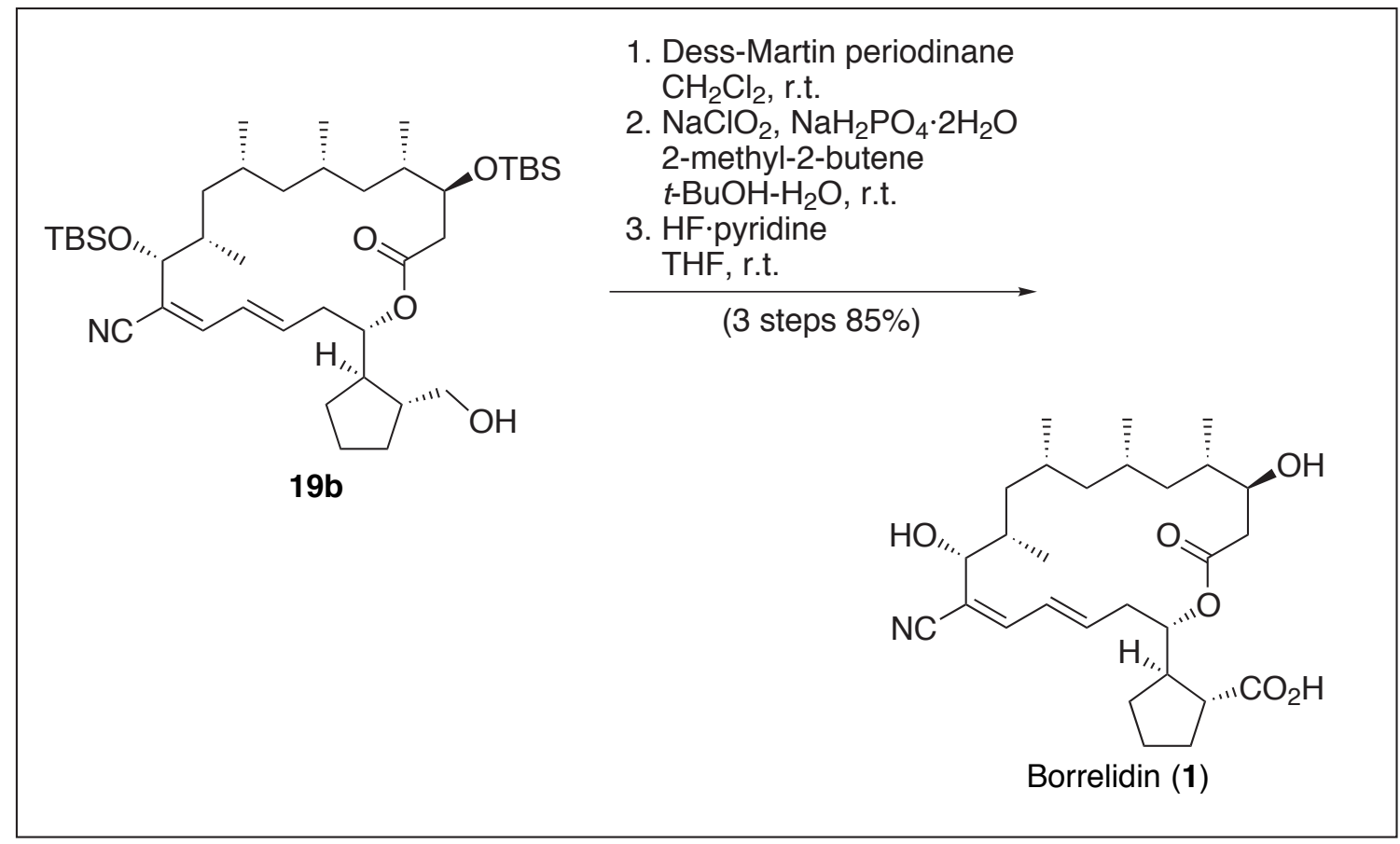

To a solution of $\mathbf{1 9 b}(8.8 \mathrm{mg}, 12.5 \mu \mathrm{mol})$ at r.t. was added Dess-Martin periodinane (10.6 mg, $25.0 \mu \mathrm{mol})$. The mixture was stirred for $30 \mathrm{~min}$. The reaction was quenched with sat. aq. $\mathrm{Na}_{2} \mathrm{~S}_{2} \mathrm{O}_{3}$ and sat. aq. $\mathrm{Na}_{2} \mathrm{~S}_{2} \mathrm{O}_{3}$ solutions, and the aqueous phase was extracted with $\mathrm{CH}_{2} \mathrm{Cl}_{2}$. The combined organic extracts were dried over anhydrous $\mathrm{Na}_{2} \mathrm{SO}_{4}$ and concentrated in vacuo. The resulting crude aldehyde was subjected to the next reaction without further purification.

The crude aldehyde was dissolved in $t$ - $\mathrm{BuOH}(250 \mu \mathrm{l})$ and $\mathrm{H}_{2} \mathrm{O}(250 \mu \mathrm{l})$ at r.t.. 2-Methyl-2butene $(7 \mu \mathrm{l}, 62.8 \mu \mathrm{mol})$, sodium phosphate $(5.8 \mathrm{mg}, 37.5 \mu \mathrm{mol})$ and sodium chlorite $(3.3 \mathrm{mg}$, $37.5 \mu \mathrm{mol}$ ) was added to the solution. After $30 \mathrm{~min}$, the reaction was poured into water and the aqueous phase was extracted with EtOAc. The combined organic extracts were dried over anhydrous $\mathrm{Na}_{2} \mathrm{SO}_{4}$ and concentrated in vacuo to give the corresponding carboxylic acid.

To a solution of the crude acid in THF $(1.0 \mathrm{ml})$ and pyridine $(1.0 \mathrm{ml})$ was added dropwise HF.pyridine $(500 \mu \mathrm{l})$. The solution was stirred for 2 days. The resulting solution was filtered through a short plug of silica gel and concentrated in vacuo. Flash chromatography (30:1 hexanes/EtOAc) afforded borrelidin (1) (5.2 mg, 3 steps $85 \%)$ as a white solid.

$[\alpha]_{\mathrm{D}}{ }^{27}=-26.7^{\circ}\left(c=0.10\right.$, EtOH); m.p. $140-142^{\circ} \mathrm{C}$; IR (KBr) 3446, 2924, 2853, 1717, 1465, 1275 , $1259 \mathrm{~cm}^{-1} ;{ }^{1} \mathbf{H}-\mathbf{N M R}\left(\mathbf{2 7 0} \mathbf{M H z} \mathbf{C D C l}_{3}\right) \delta 0.73\left(\mathrm{~m}, 1 \mathrm{H}, 1 / 2 \mathbf{C H}_{2}\right), 0.80\left(\mathrm{~d}, 3 \mathrm{H}, J=6.3 \mathrm{~Hz}, \mathrm{CH}_{3}\right)$, $0.83\left(\mathrm{~d}, 3 \mathrm{H}, J=7.2 \mathrm{~Hz}, \mathrm{CH}_{3}\right), 0.84\left(\mathrm{~d}, 3 \mathrm{H}, J=6.6 \mathrm{~Hz}, \mathrm{CH}_{3}\right), 1.05$ (d, 3H, J = 6.6 Hz, CH $\mathbf{C H}_{3}, 0.89$ - 
$1.44\left(\mathrm{~m}, 6 \mathrm{H}, \mathrm{CH}_{2} \times 3\right), 1.50-1.71(\mathrm{~m}, 3 \mathrm{H}, \mathrm{CH} \times 3), 1.75-2.11\left(\mathrm{~m}, 6 \mathrm{H}, \mathrm{CH}_{2} \times 2,1 / 2 \mathrm{CH}_{2}\right.$ and $\left.\mathrm{CH}\right)$, $2.32\left(\mathrm{dd}, 1 \mathrm{H}, J=16.8,2.3 \mathrm{~Hz}, 1 / 2 \mathrm{CH}_{2}\right), 2.44\left(\mathrm{dd}, 1 \mathrm{H}, J=16.8,9.9 \mathrm{~Hz}, 1 / 2 \mathbf{C H}_{2}\right), 2.49-2.78(\mathrm{~m}$, $4 \mathrm{H}, \mathrm{CH}_{2}$ and $\left.\mathbf{C H} \times 2\right), 3.87\left(\mathrm{dt}, 1 \mathrm{H}, J=9.9,2.3 \mathrm{~Hz}, \mathrm{C}_{16}-\mathbf{H}\right), 4.11\left(\mathrm{~d}, 1 \mathrm{H}, J=9.6 \mathrm{~Hz}, \mathrm{C}_{8}-\mathbf{H}\right), 4.98$ (dt, $\left.1 \mathrm{H}, J=10.6,3.3 \mathrm{~Hz}, \mathrm{C}_{2}-\mathbf{H}\right), 6.20$ (ddd, $\left.1 \mathrm{H}, J=14.5,9.2,5.3 \mathrm{~Hz}, \mathrm{C}_{4}-\mathbf{H}\right), 6.39$ (dd, $1 \mathrm{H}, J=$ 14.5, 11.2 Hz, $\left.\mathrm{C}_{5}-\mathbf{H}\right), 6.83\left(\mathrm{~d}, 1 \mathrm{H}, J=11.2 \mathrm{~Hz}, \mathrm{C}_{6}-\mathbf{H}\right) ;{ }^{13} \mathbf{C}-\mathbf{N M R}\left(\mathbf{6 7 . 5} \mathbf{~ M H z}, \mathbf{C D C l}_{3}\right) \delta 14.9$, $16.9,18.2$, 20.1, 25.2, 26.2, 27.1, 29.7, 31.2, 35.2, 35.6, 35.9, 37.4, 39.3, 43.0, 45.8, 47.8, 48.4, 69.8, 73.1, 77.2, 115.9, 118.2, 127.0, 138.5, 144.0, 172.2, 180.6; HRMS [FAB, m-NBA] calcd for $\mathrm{C}_{28} \mathrm{H}_{43} \mathrm{O}_{6} \mathrm{Na}\left[\mathrm{M}+\mathrm{Na}^{+}\right]: 512.2988$; found: 512.2978 . 

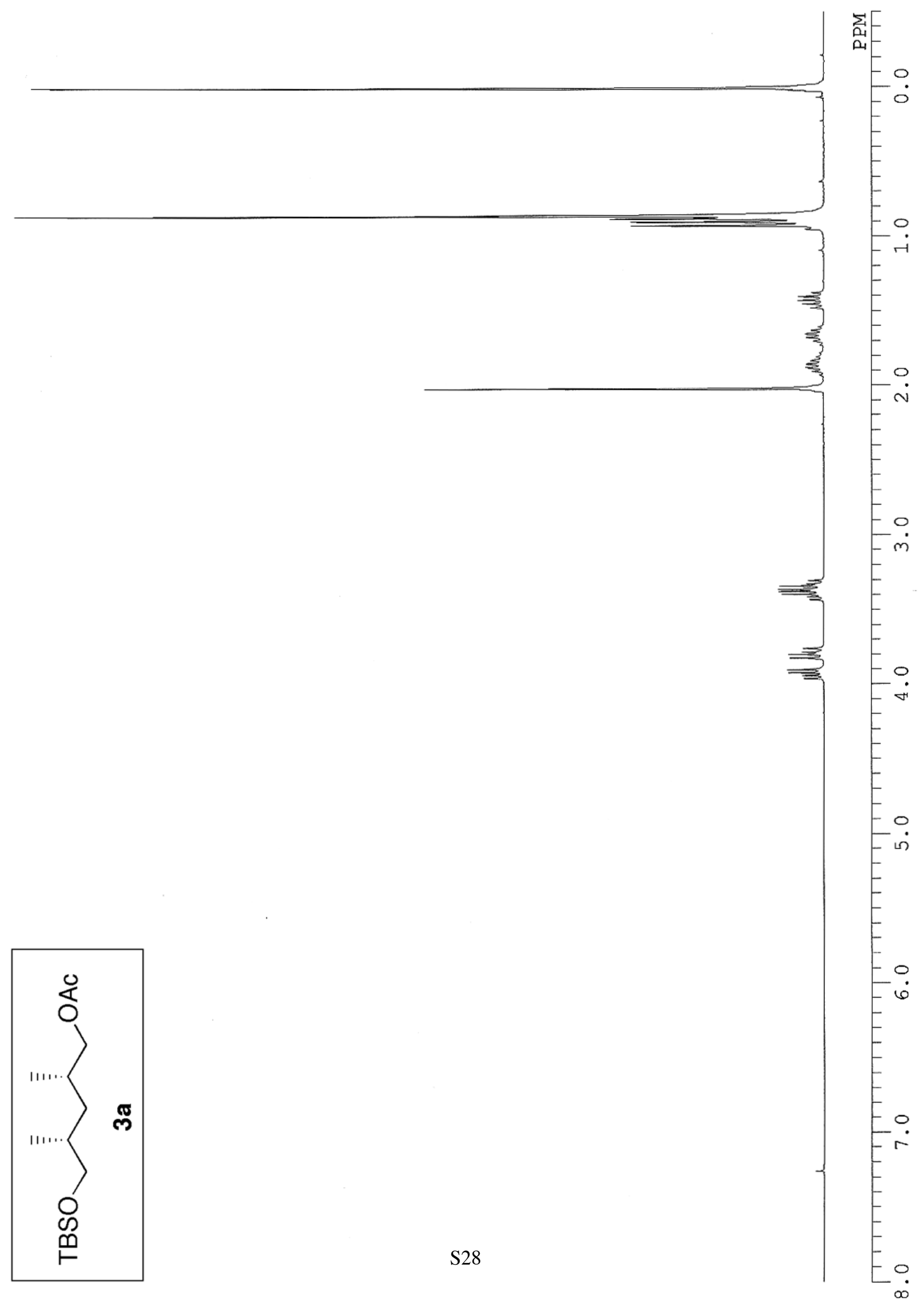


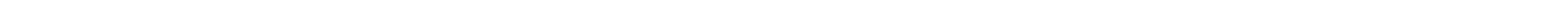




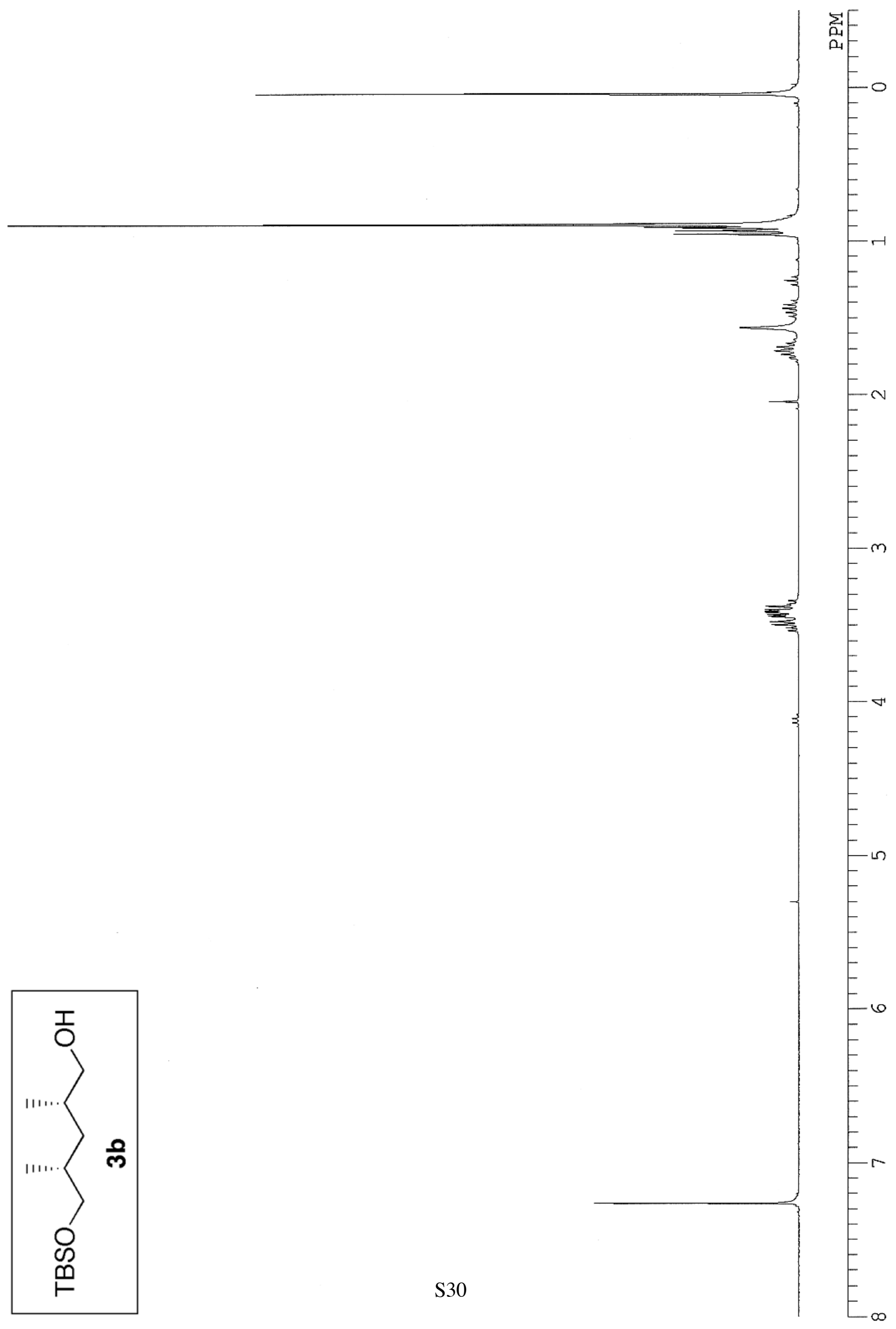




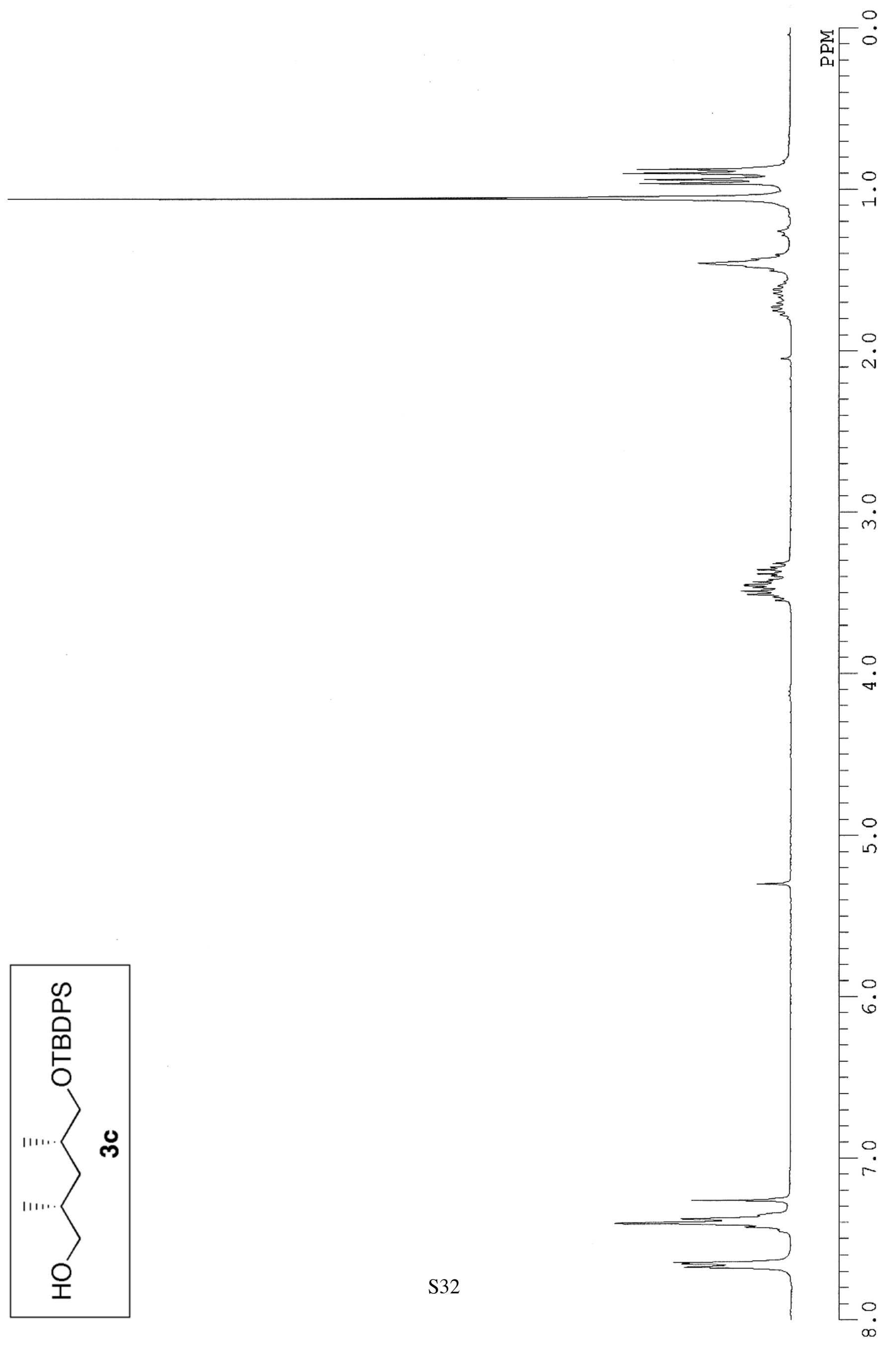




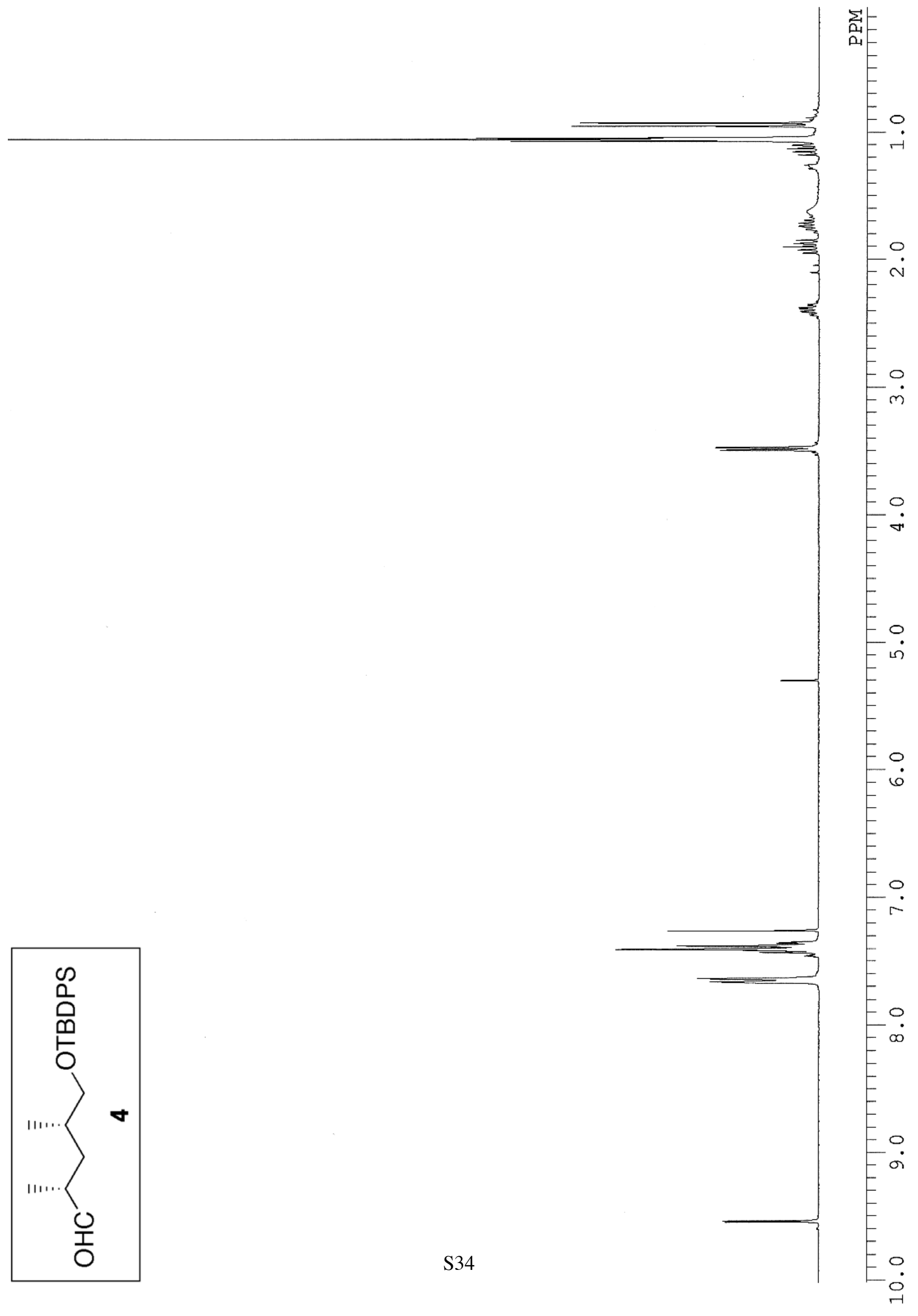




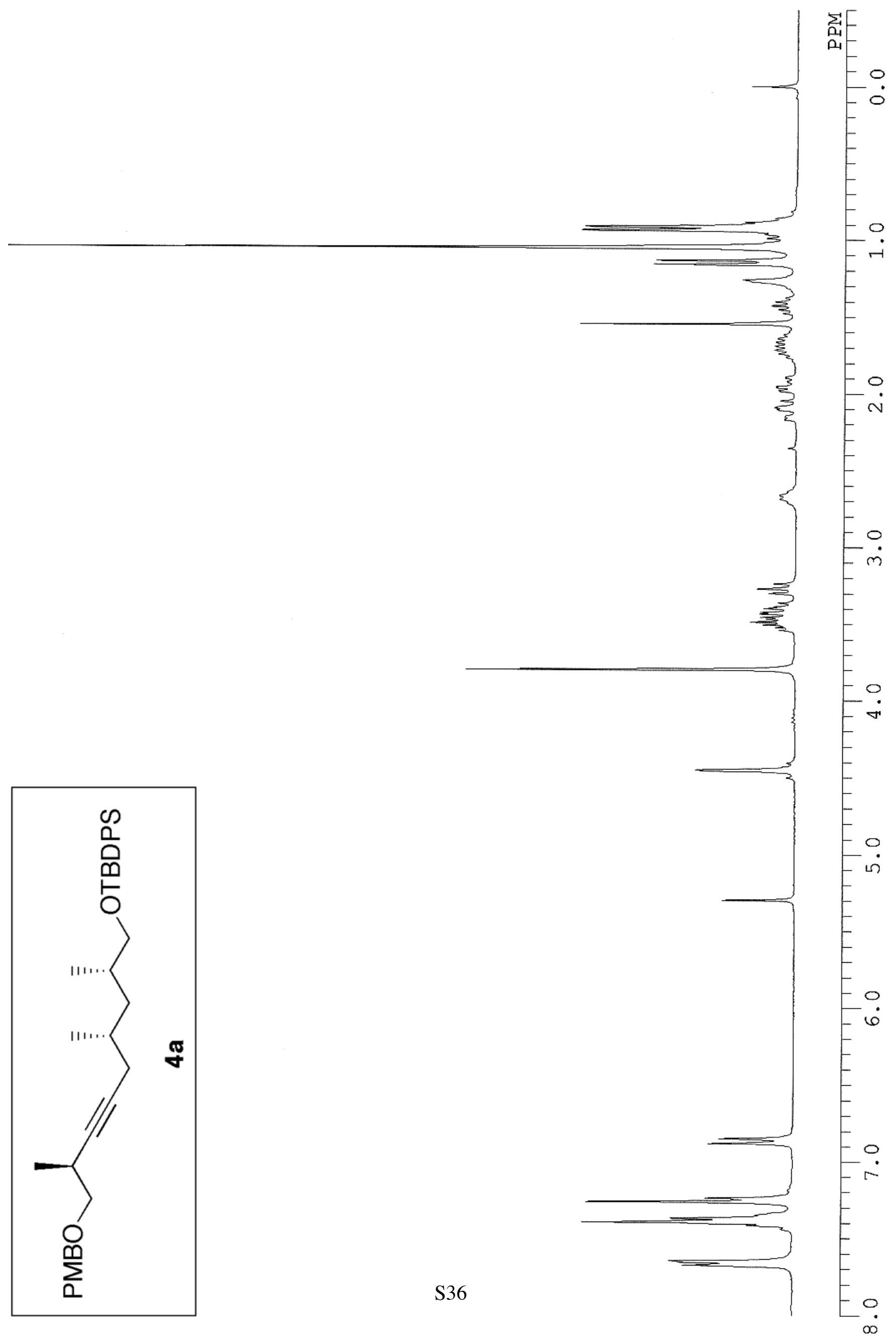




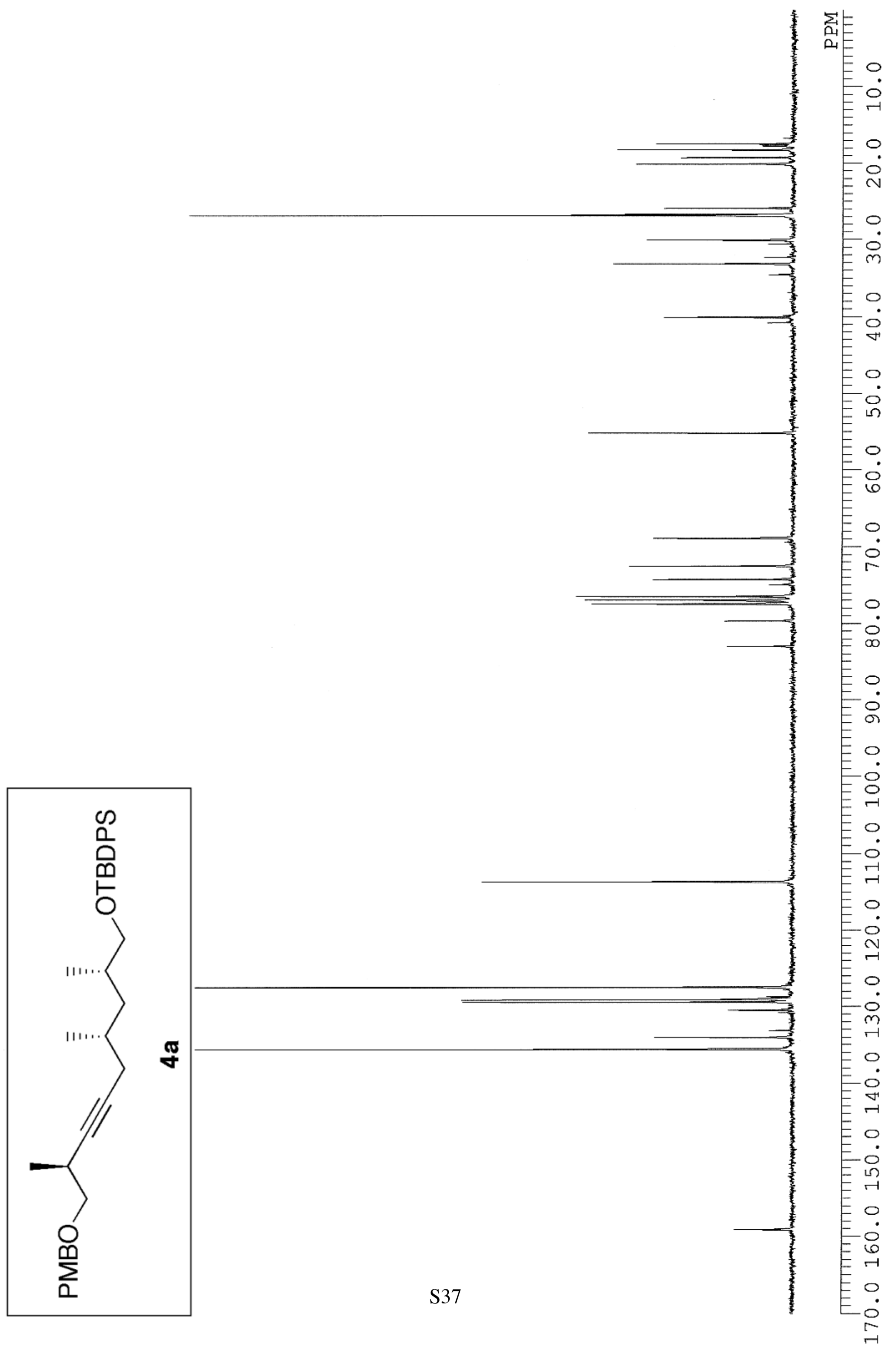




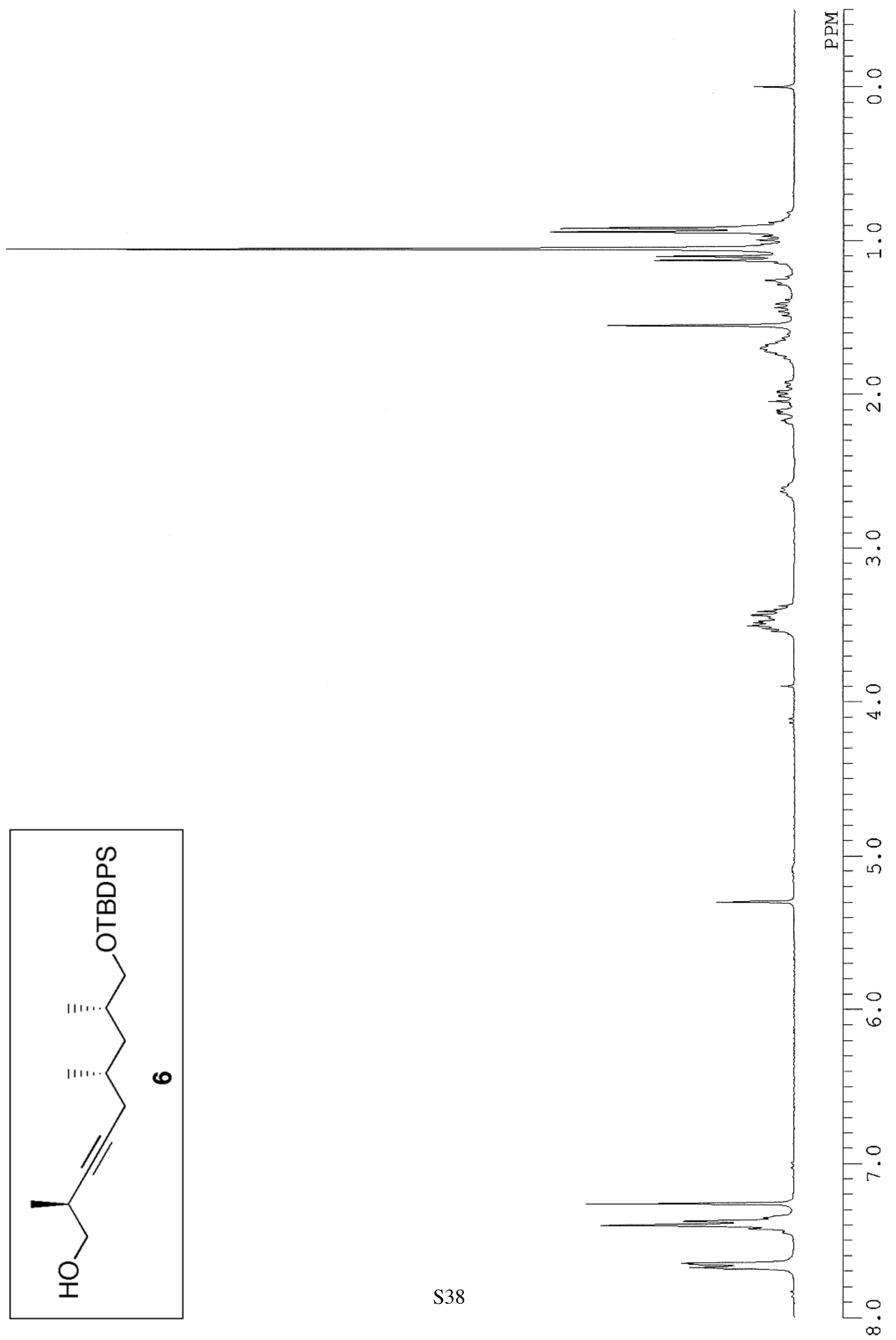




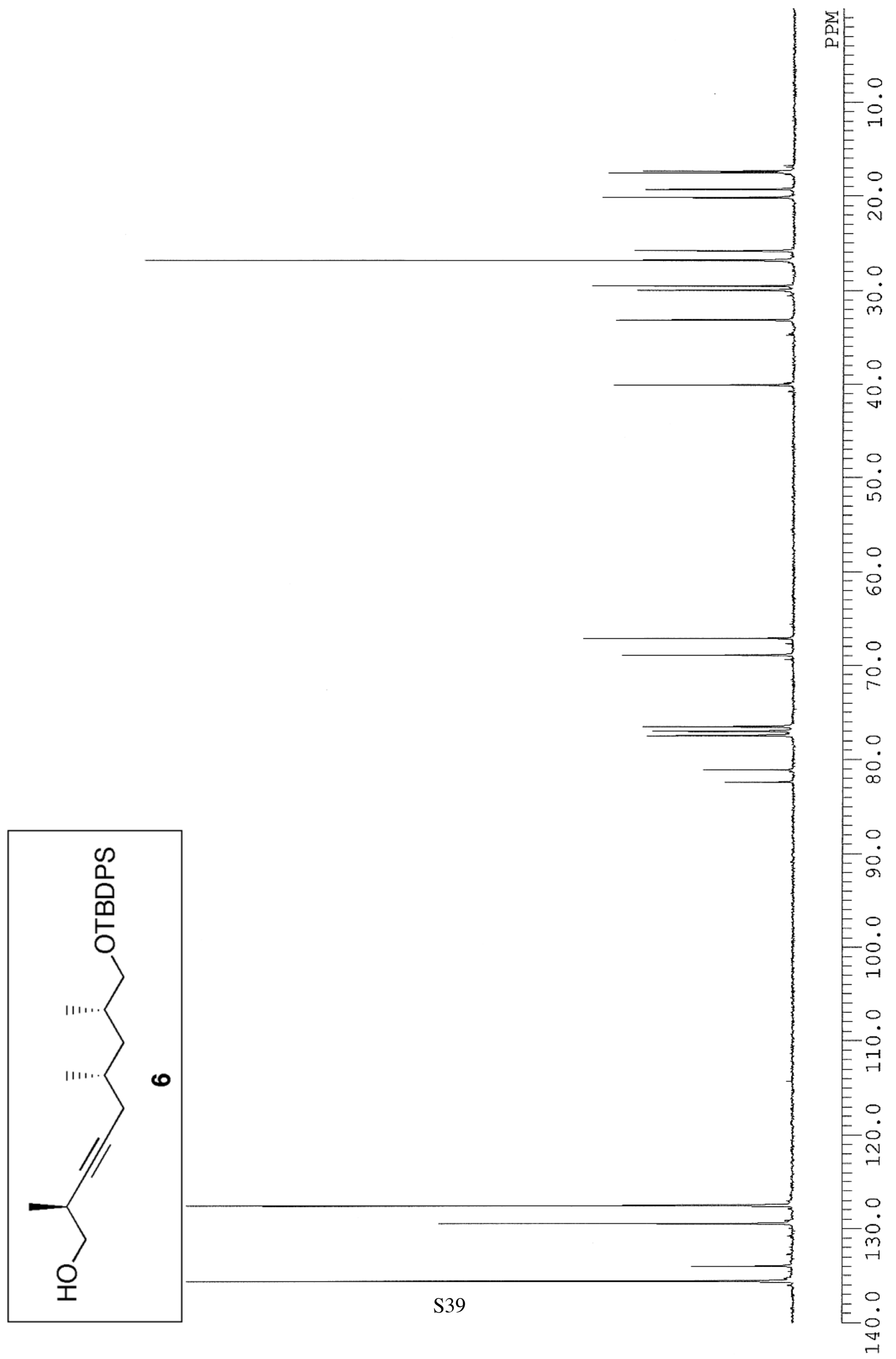




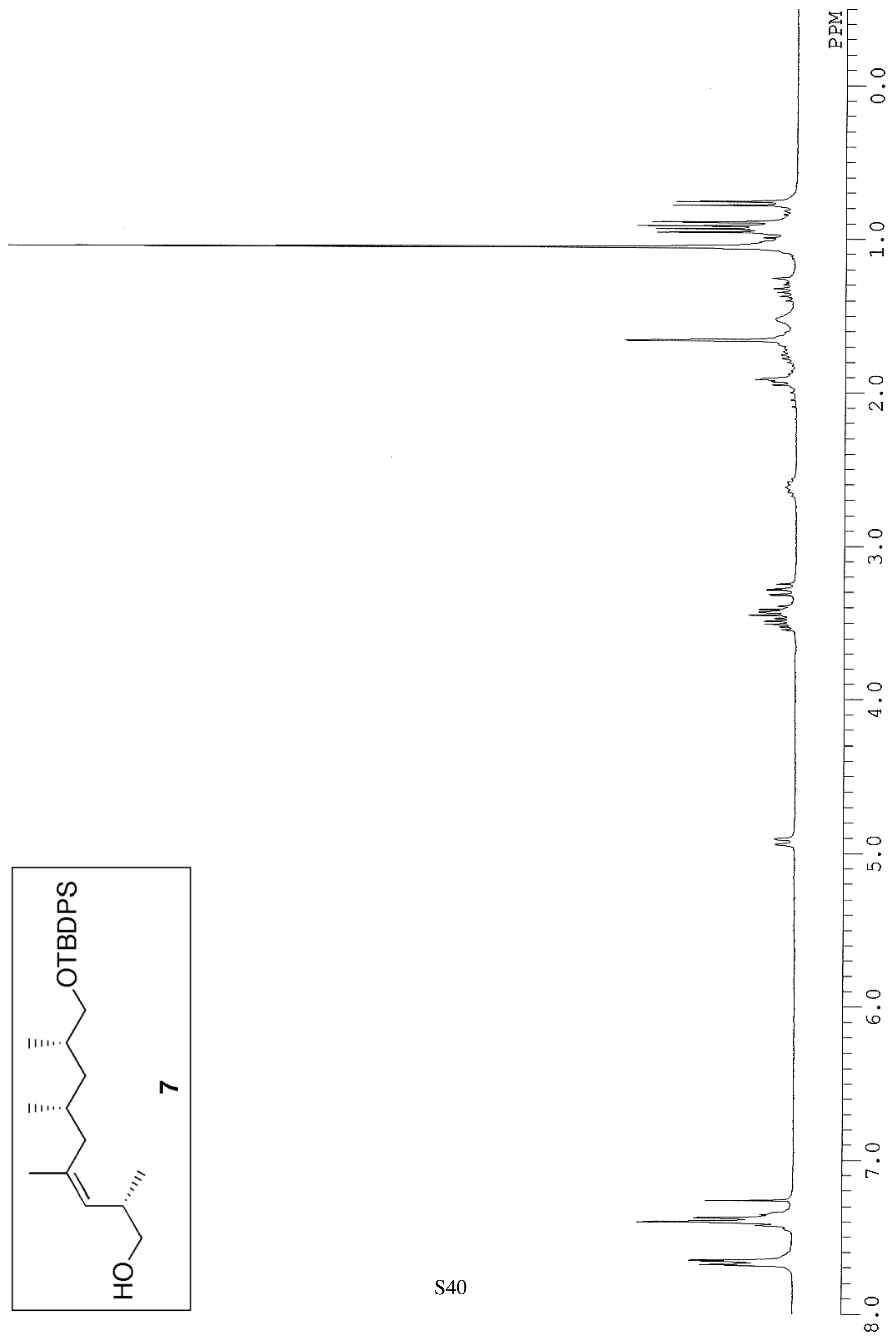




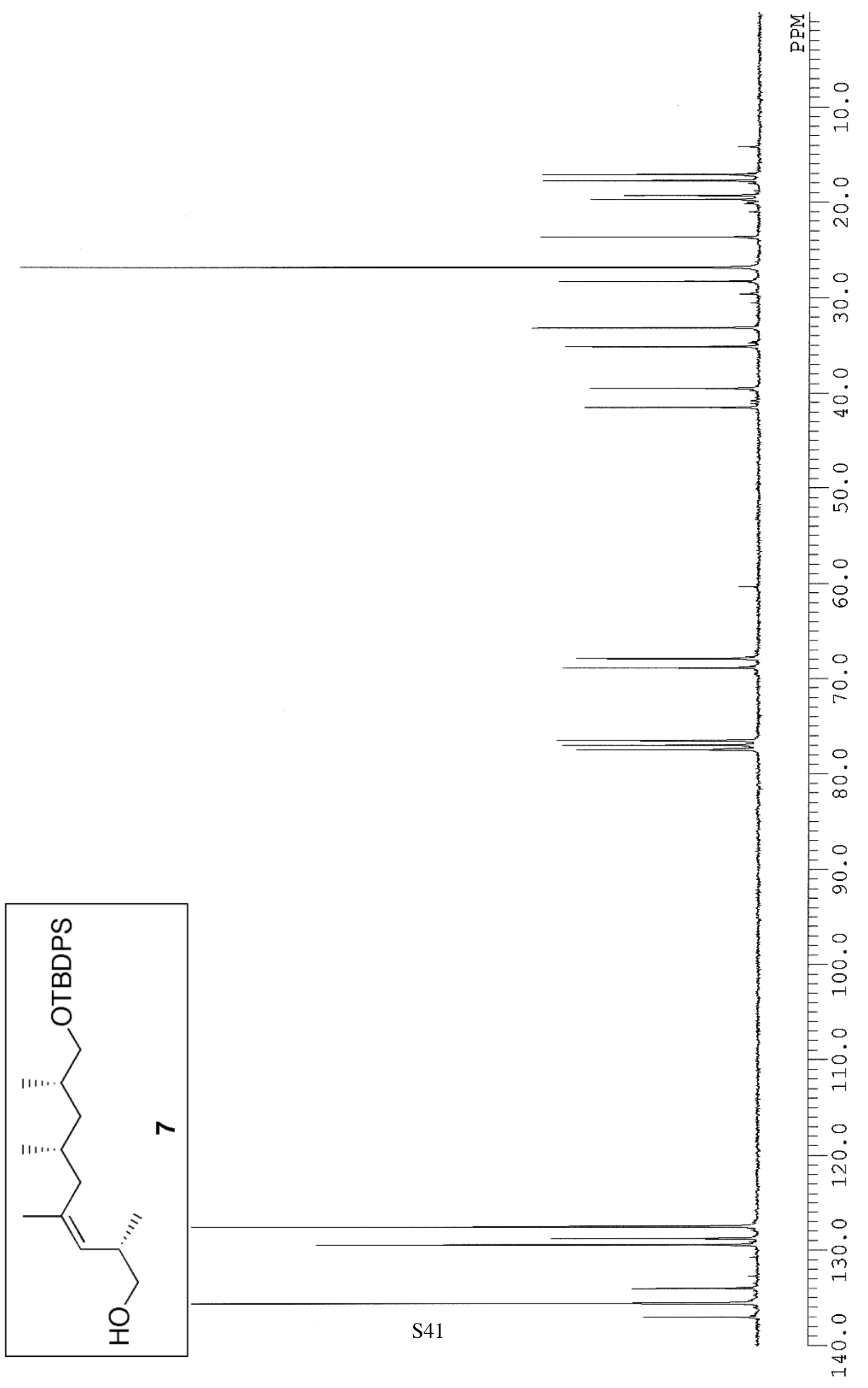




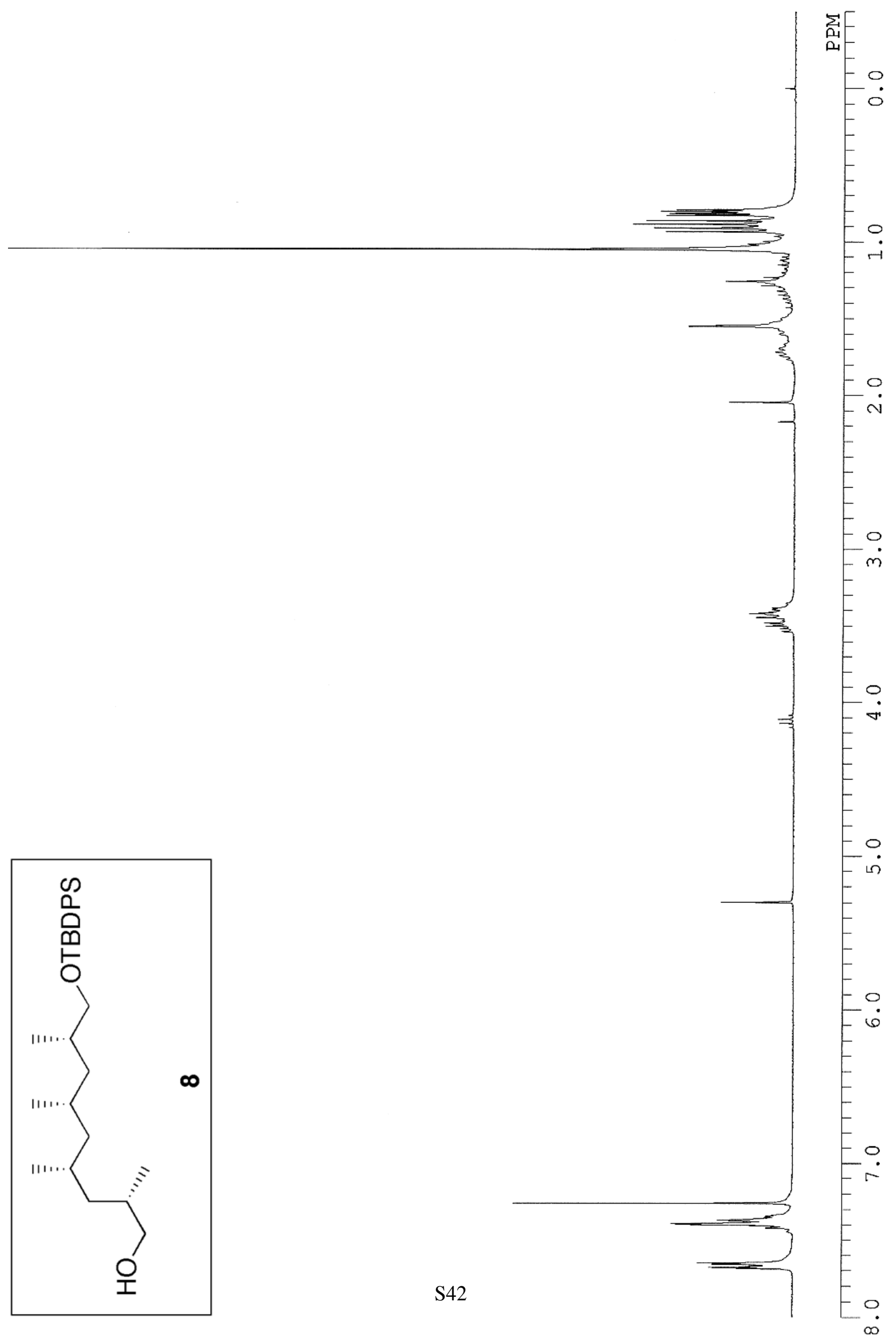




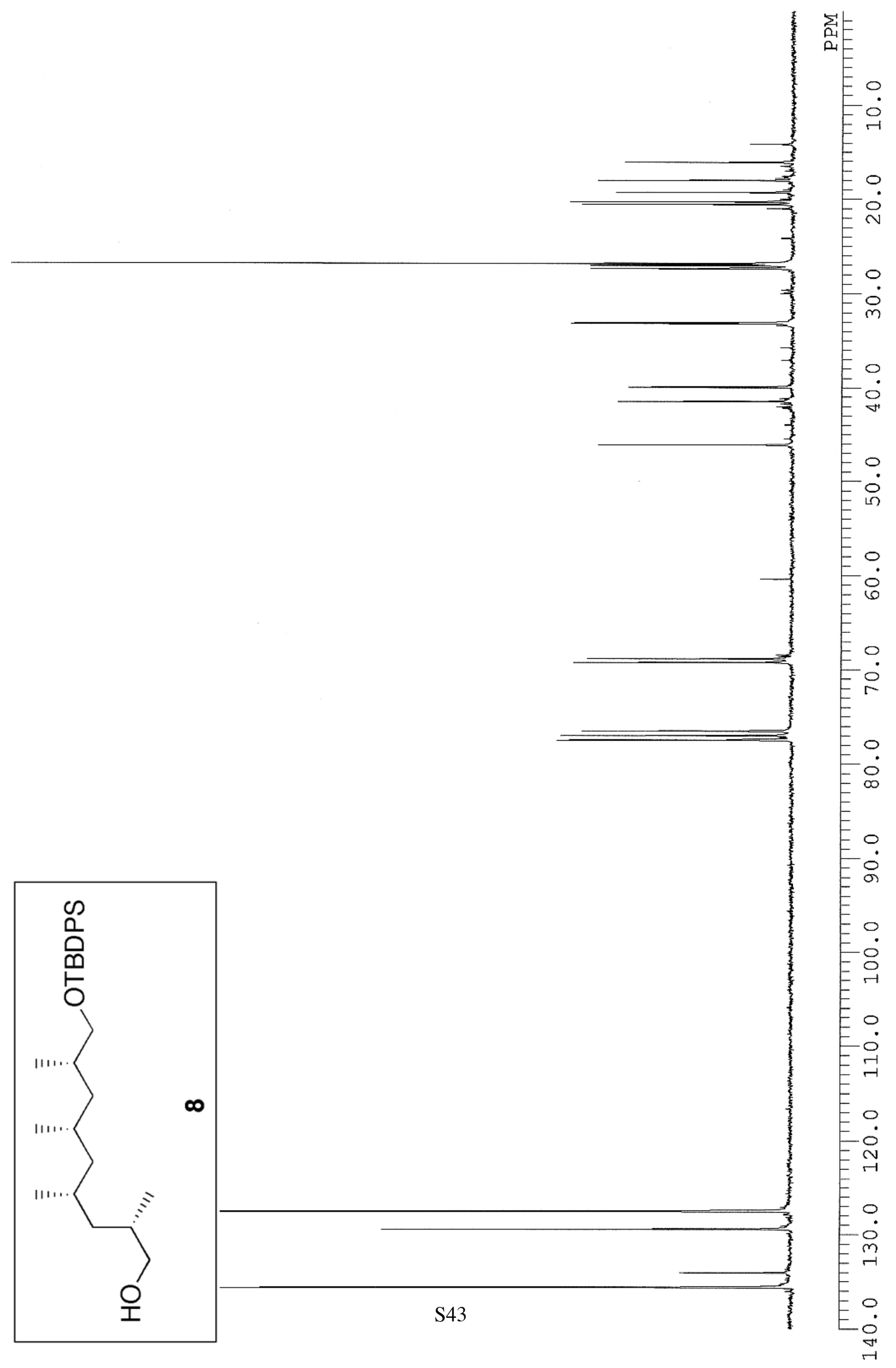



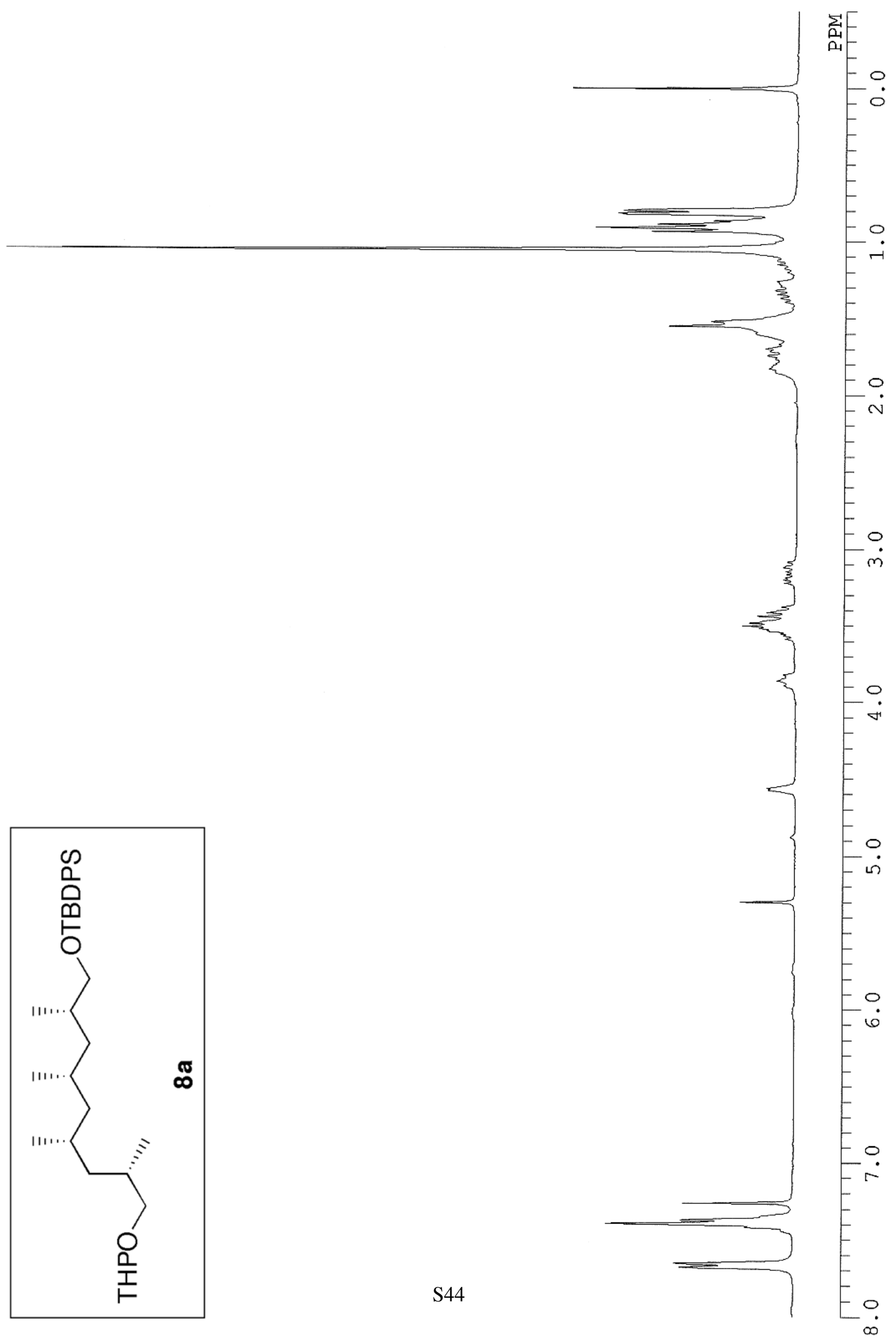


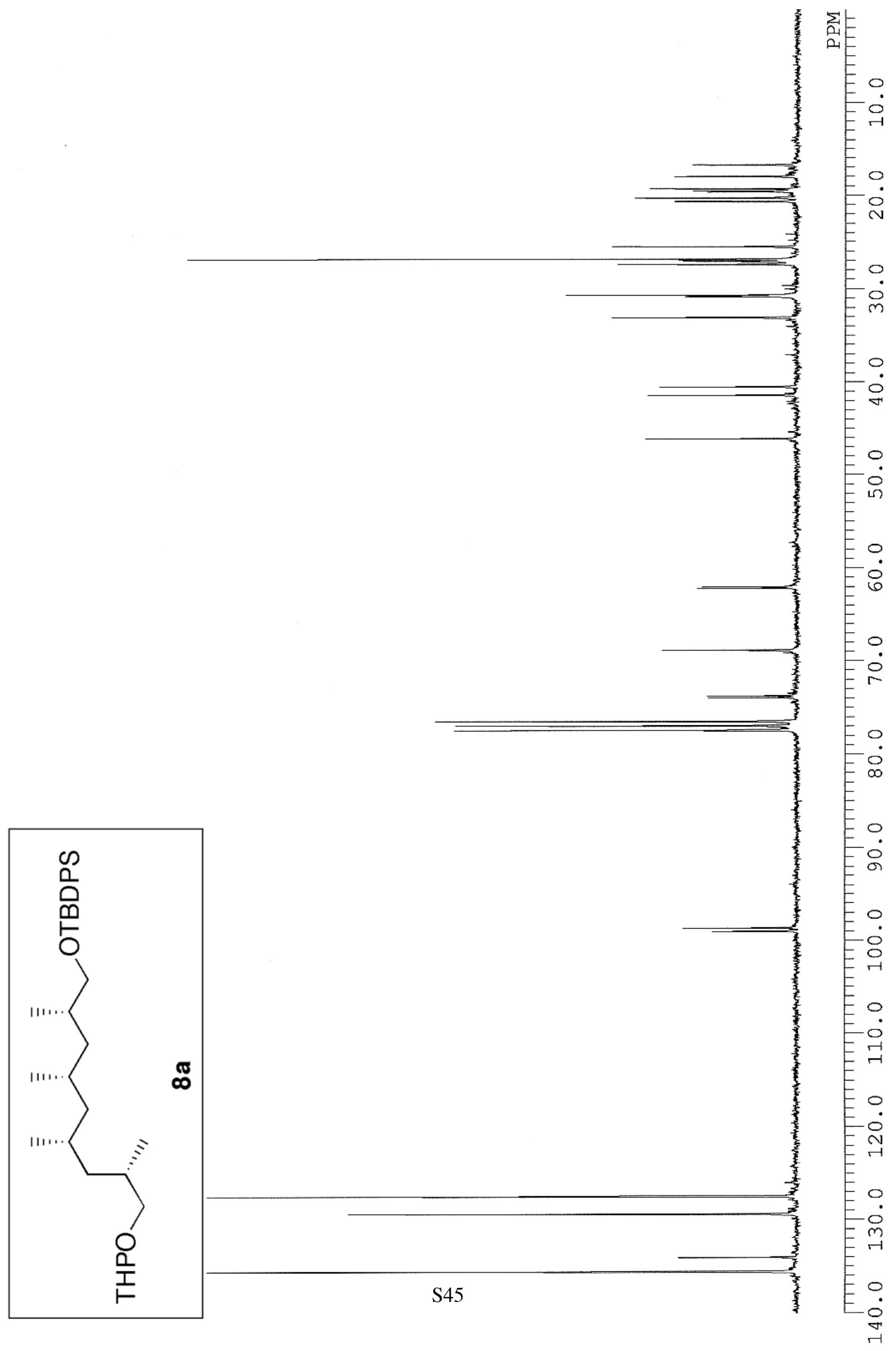




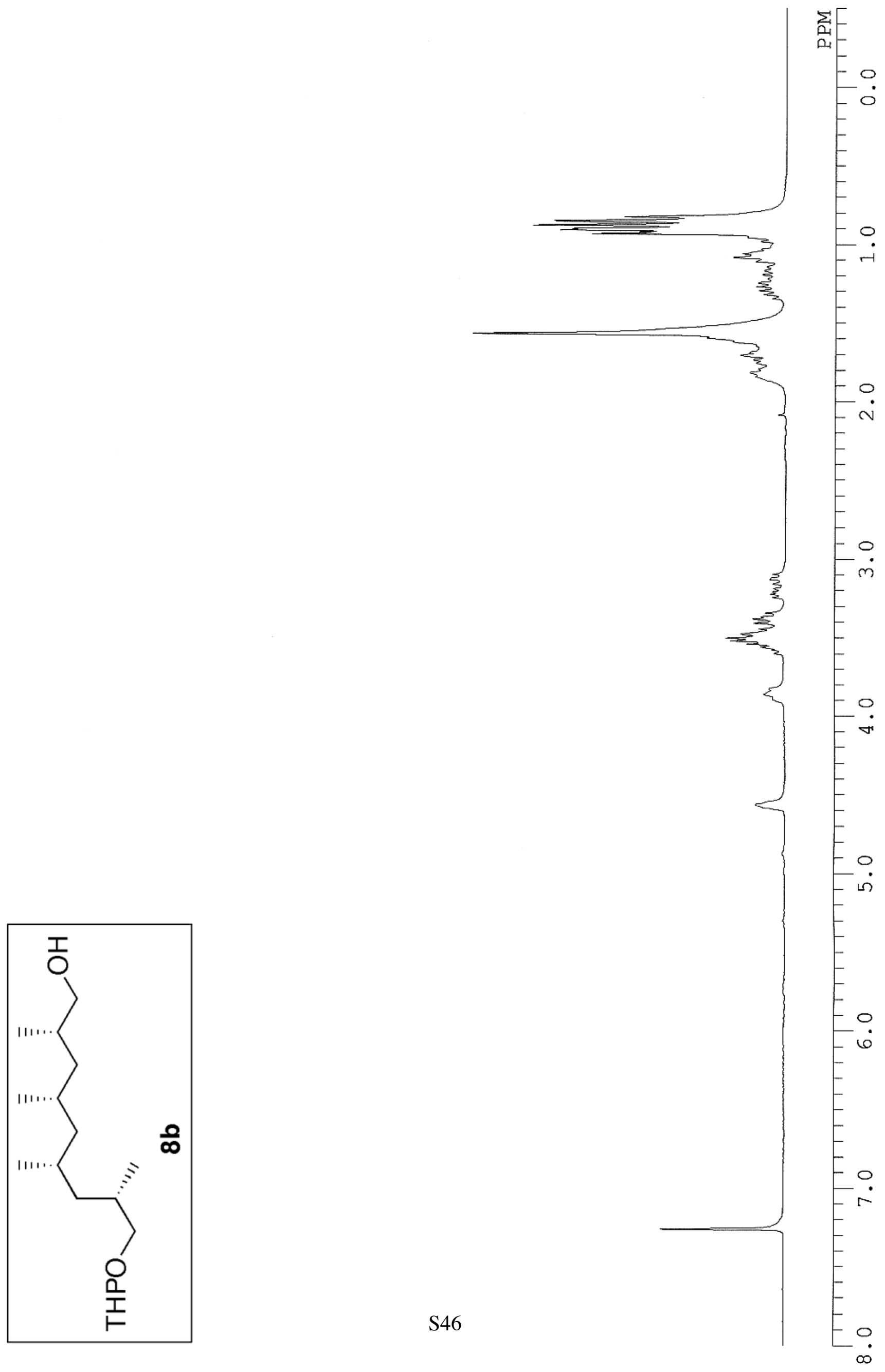




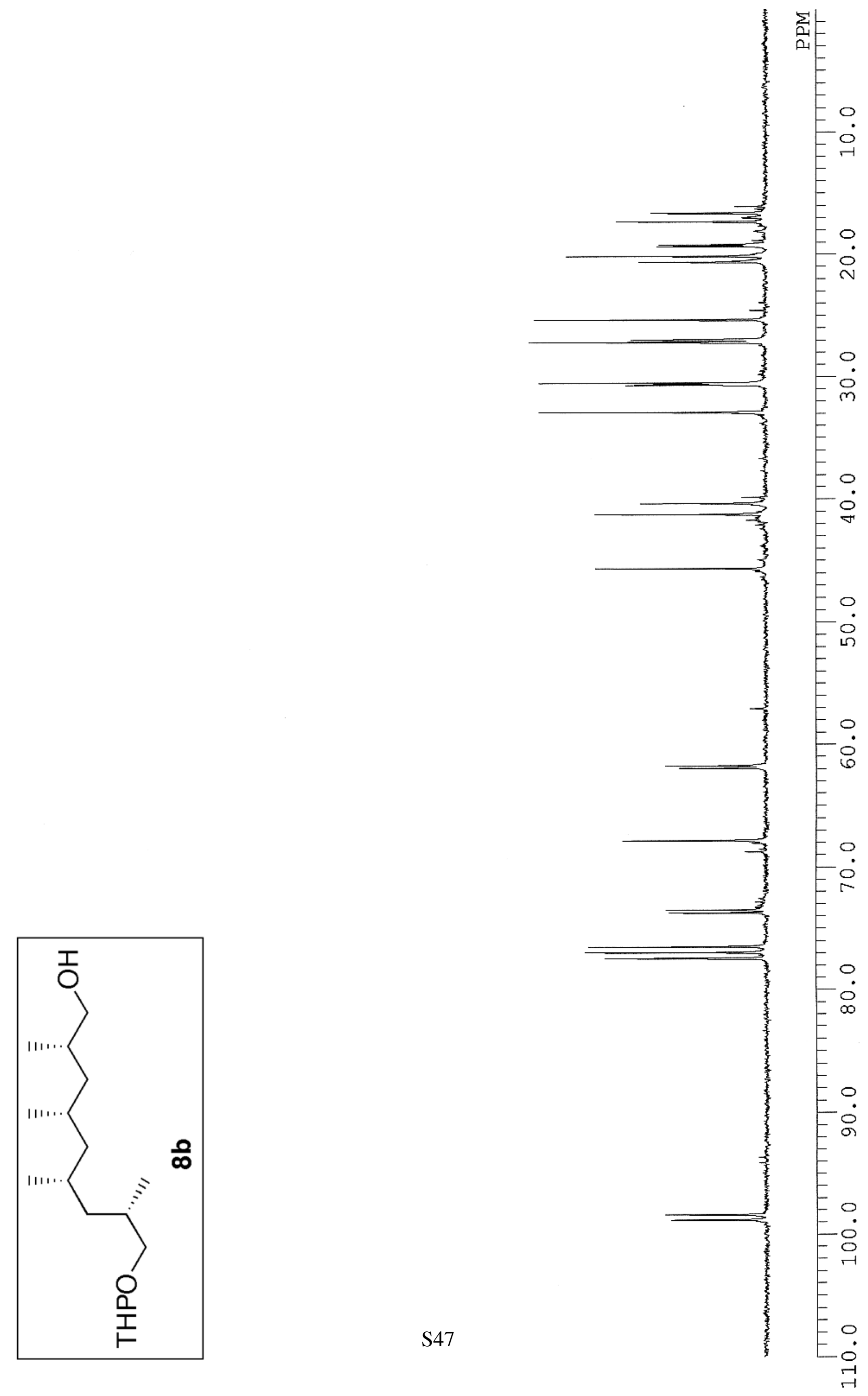




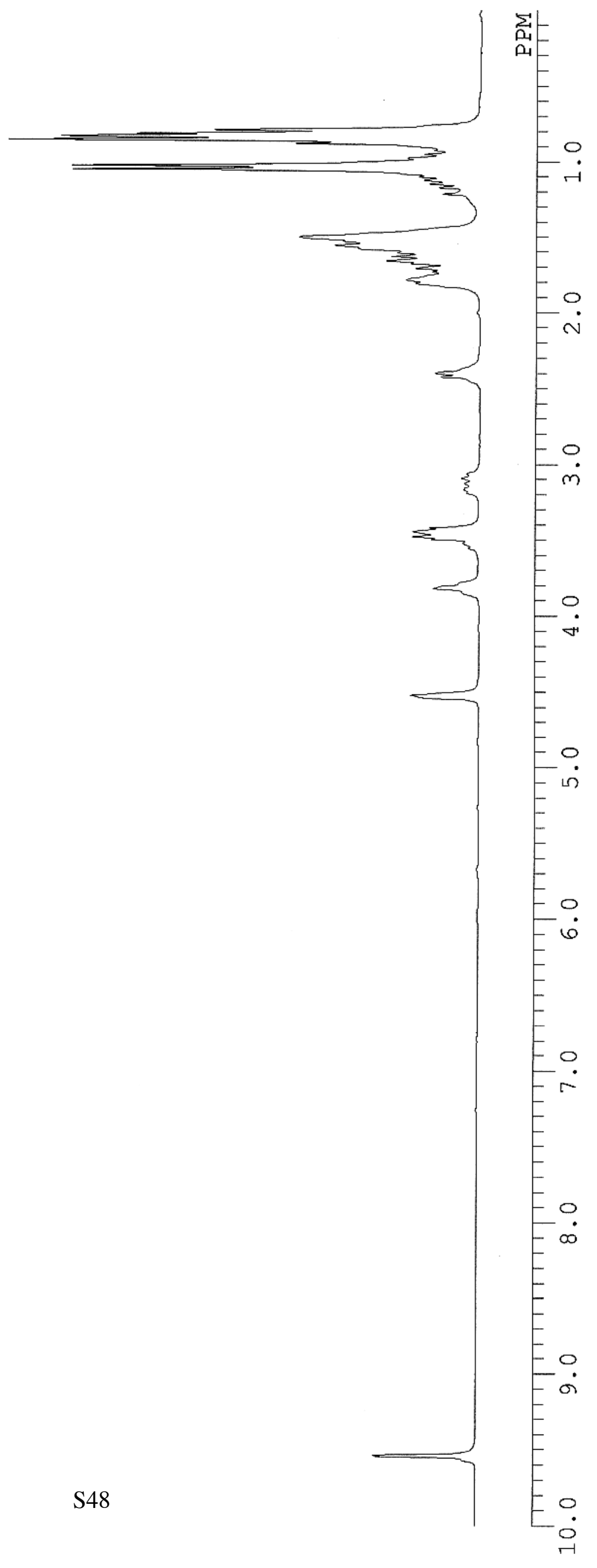



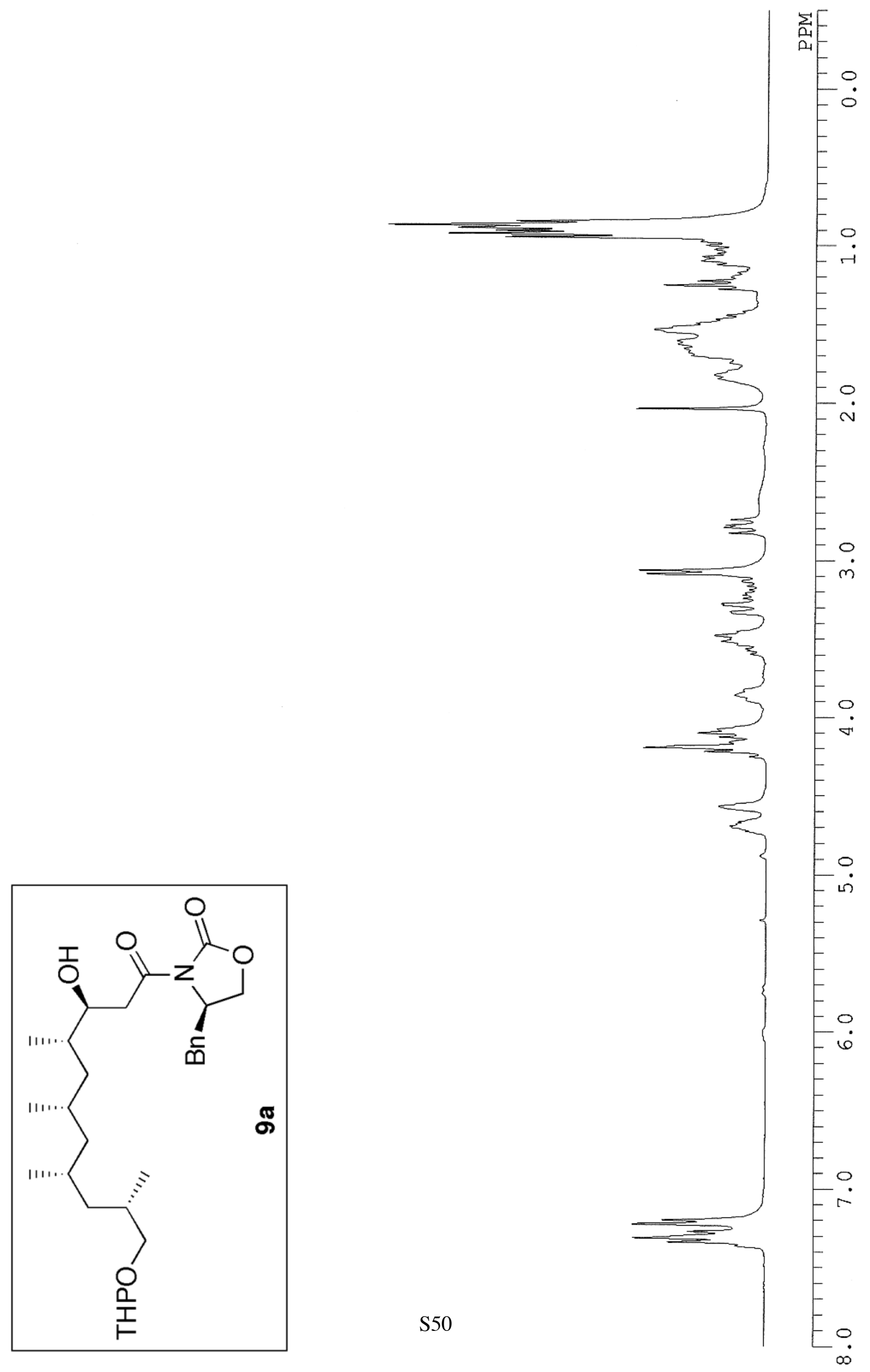


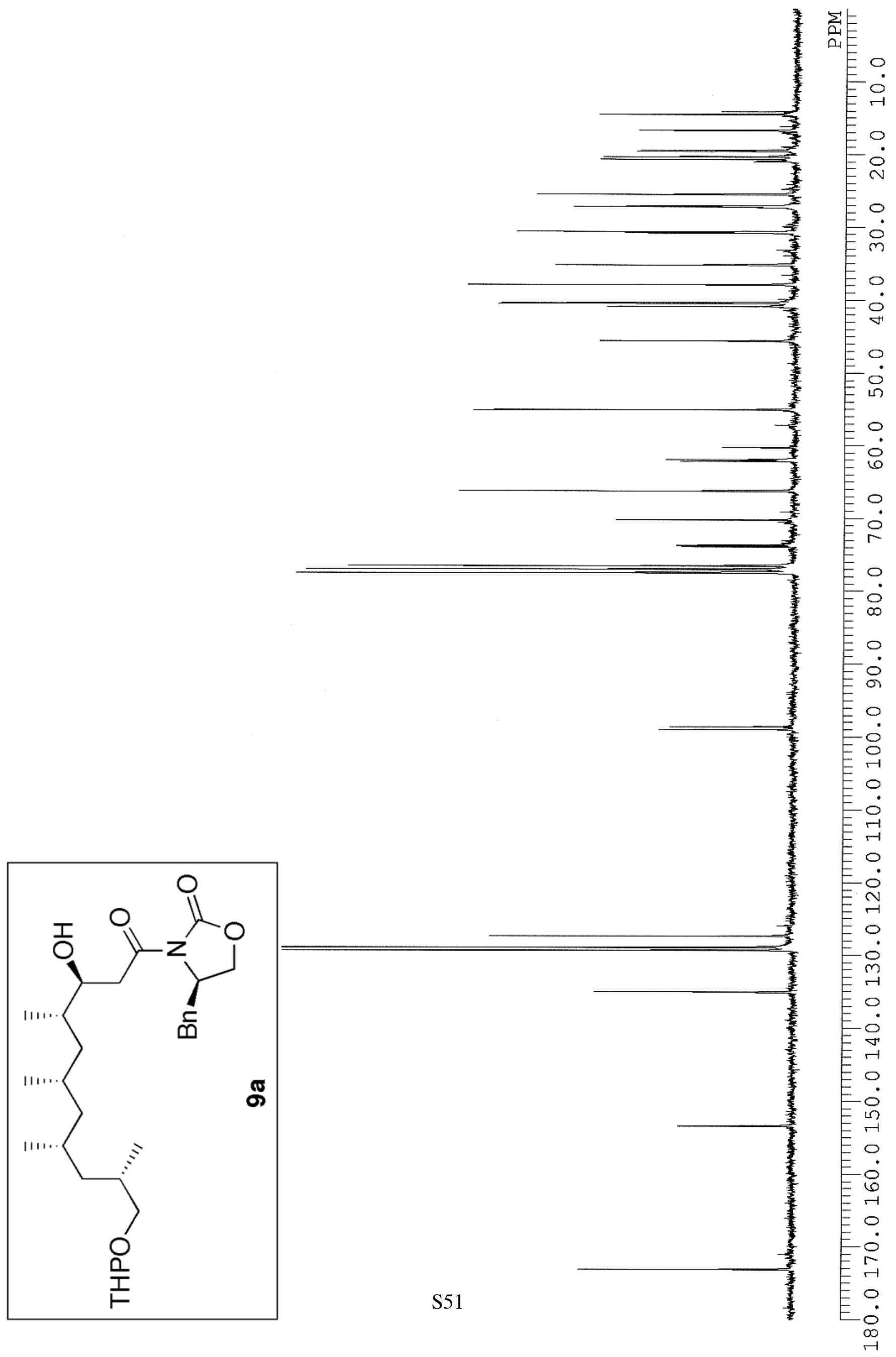




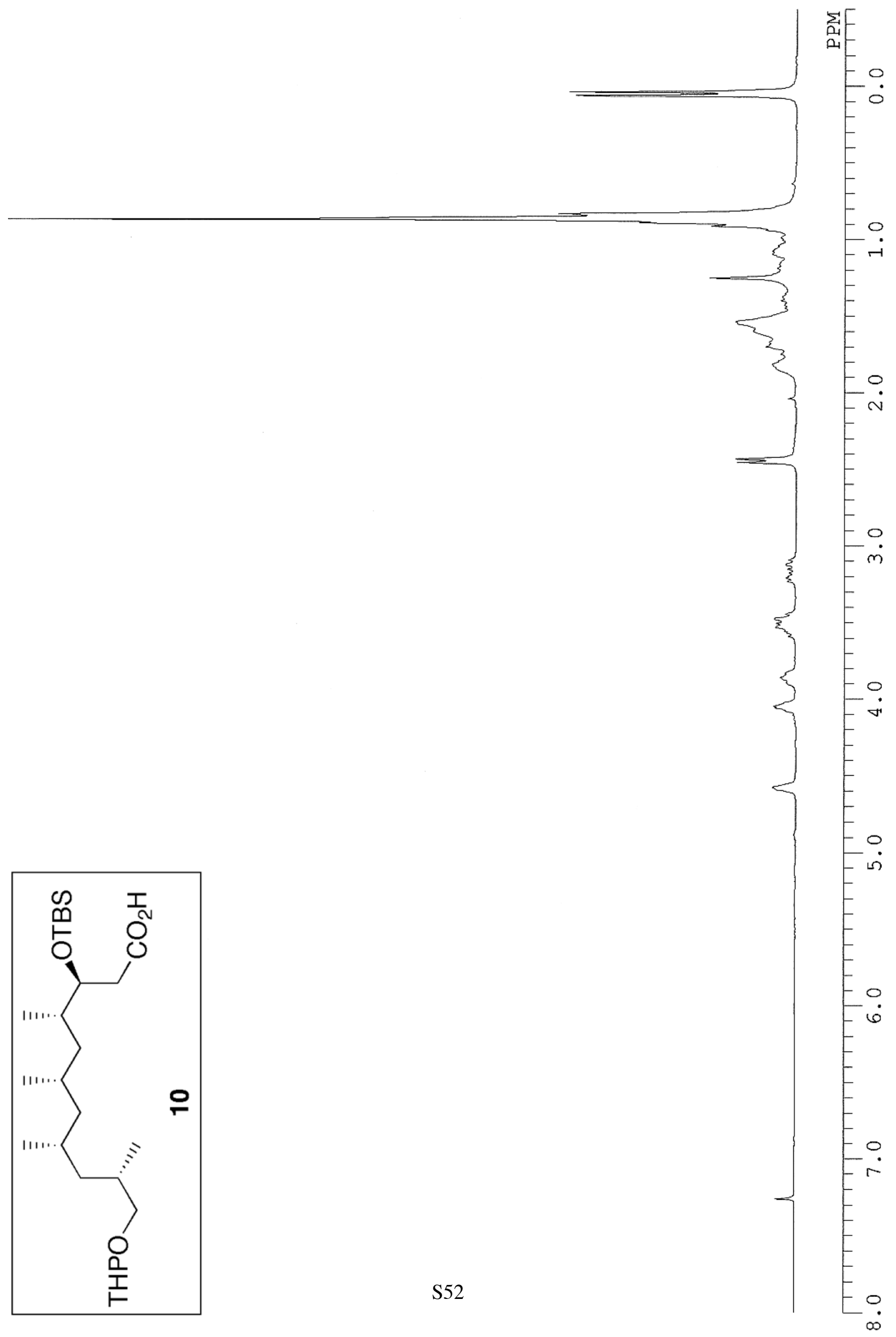




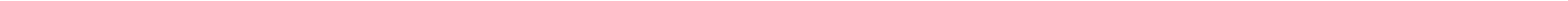




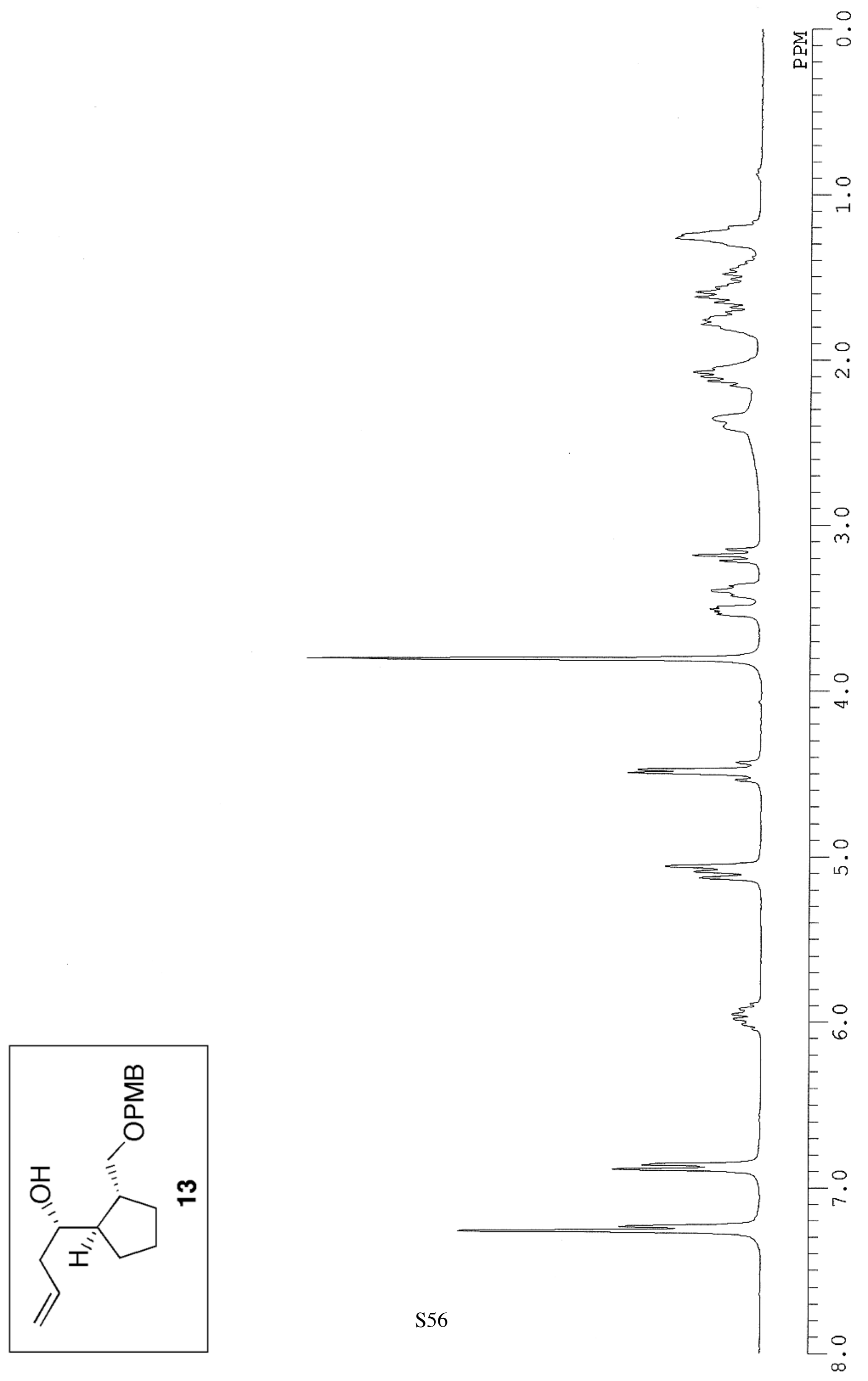




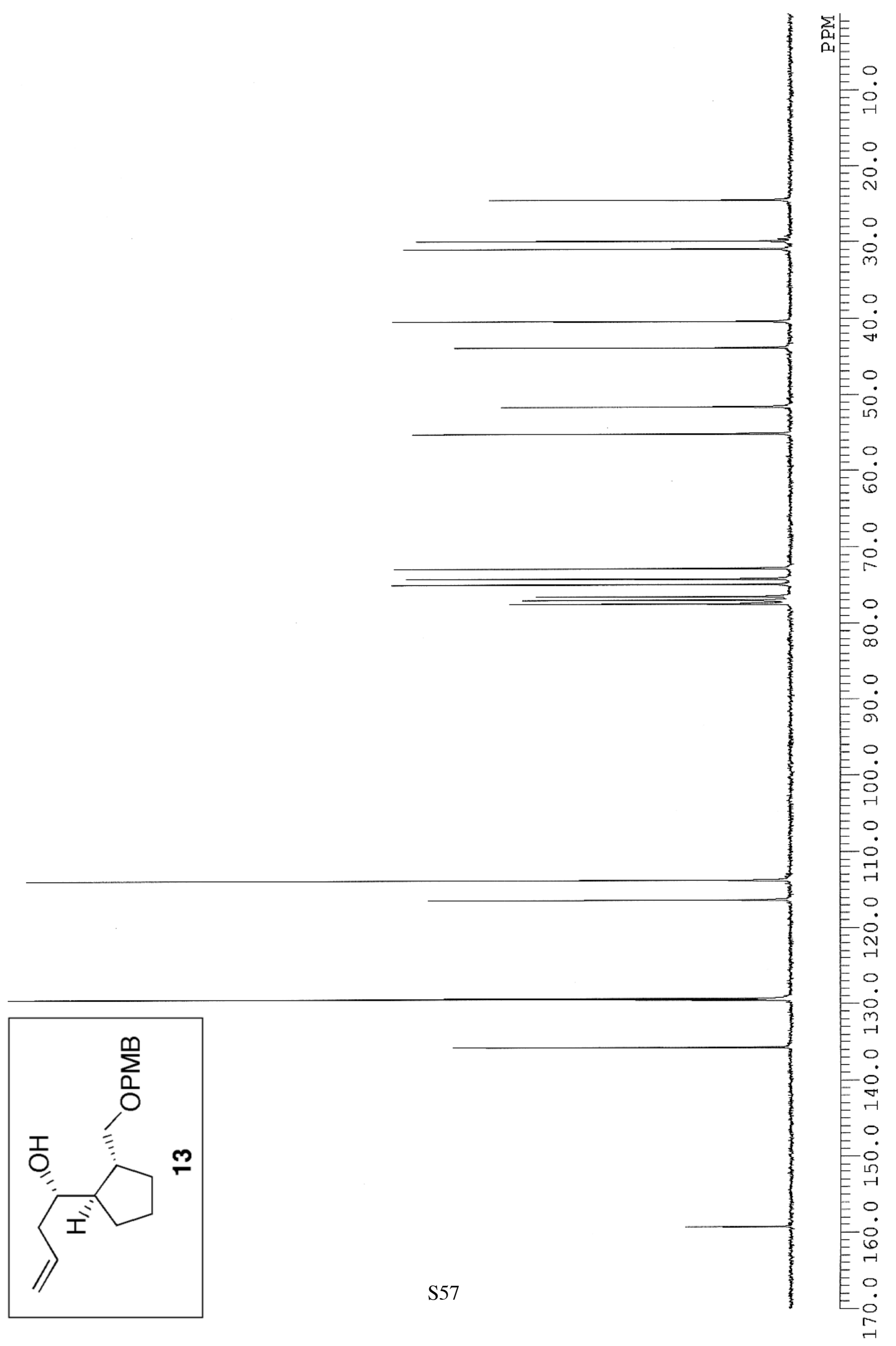




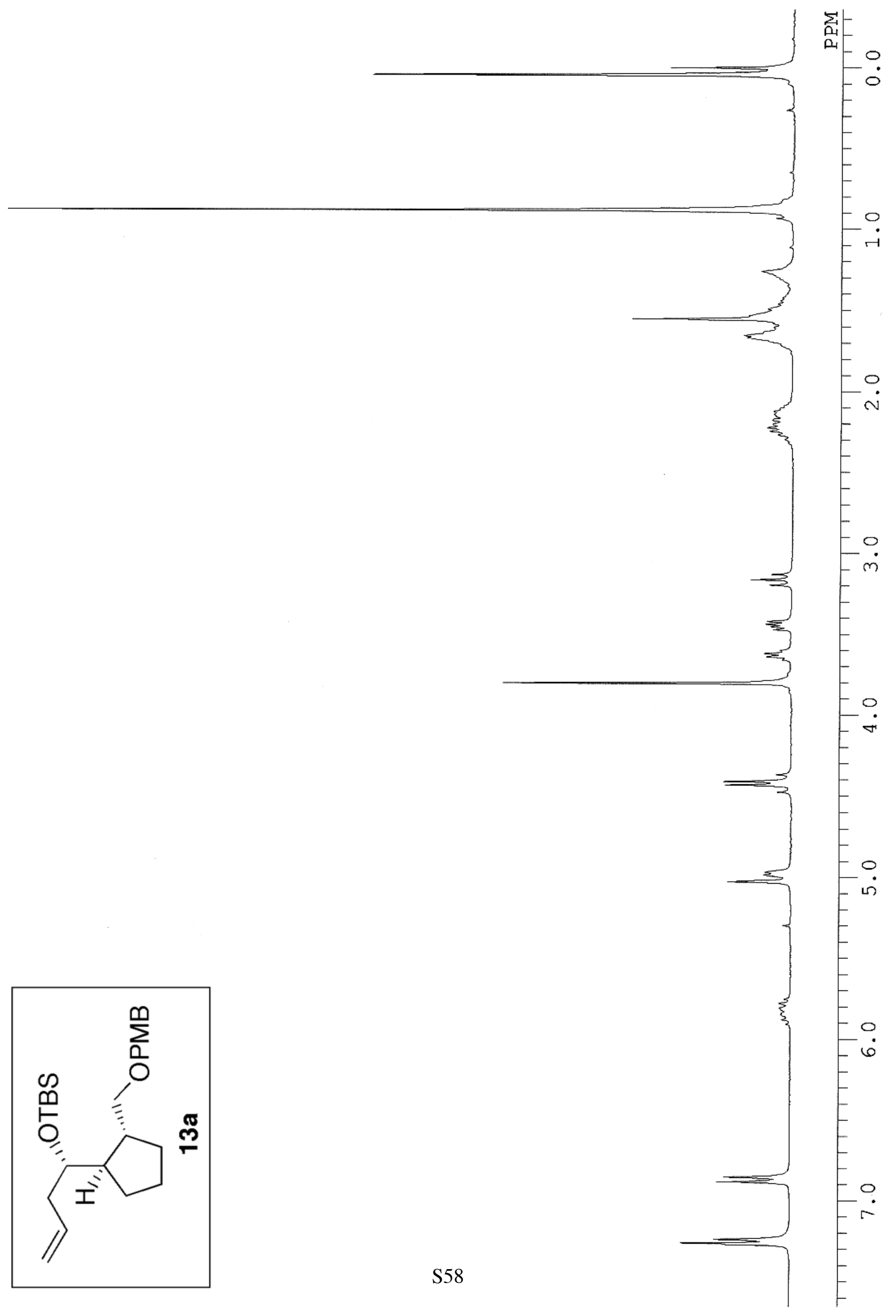




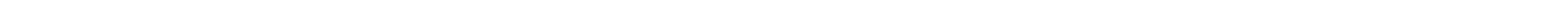




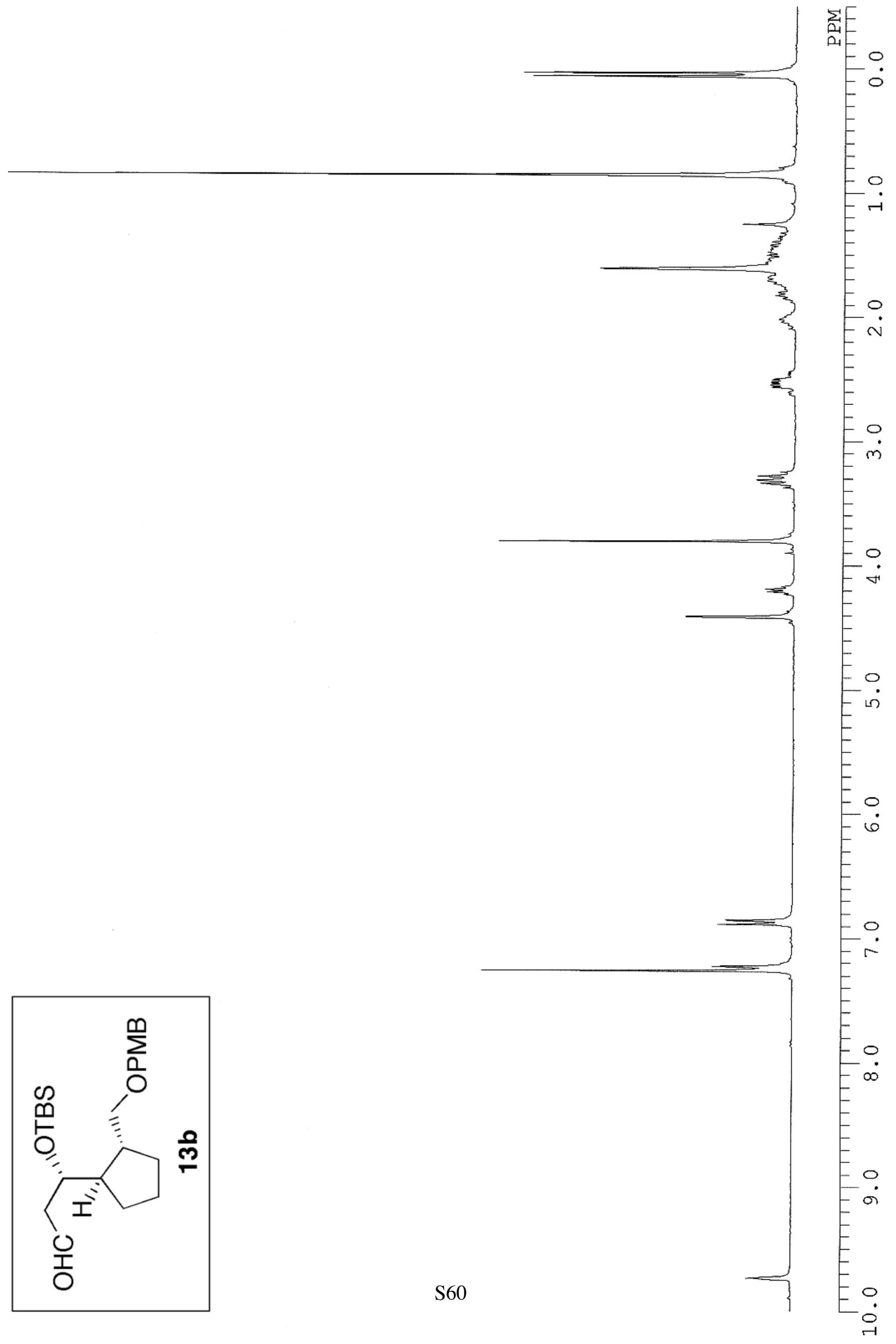




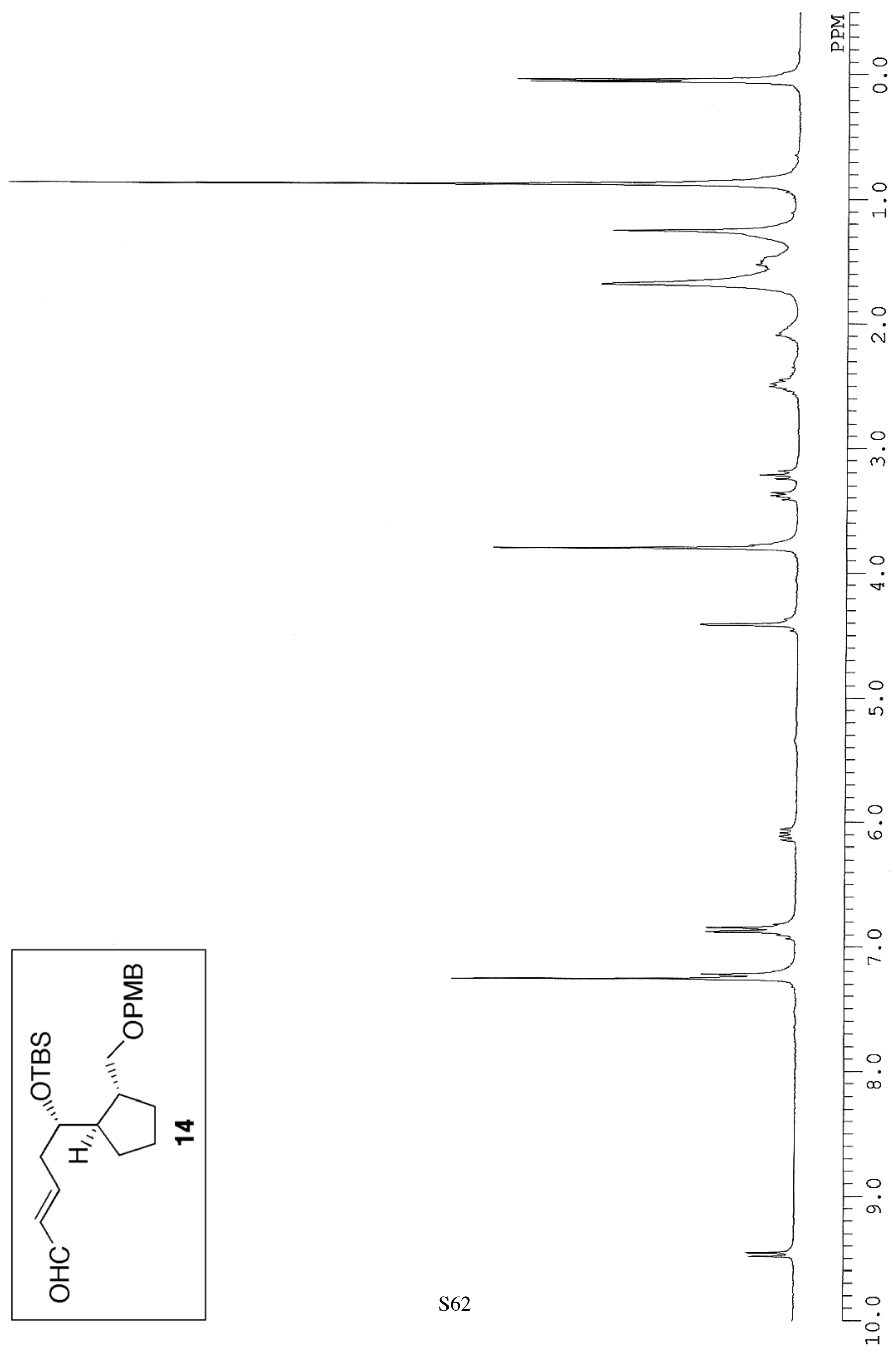




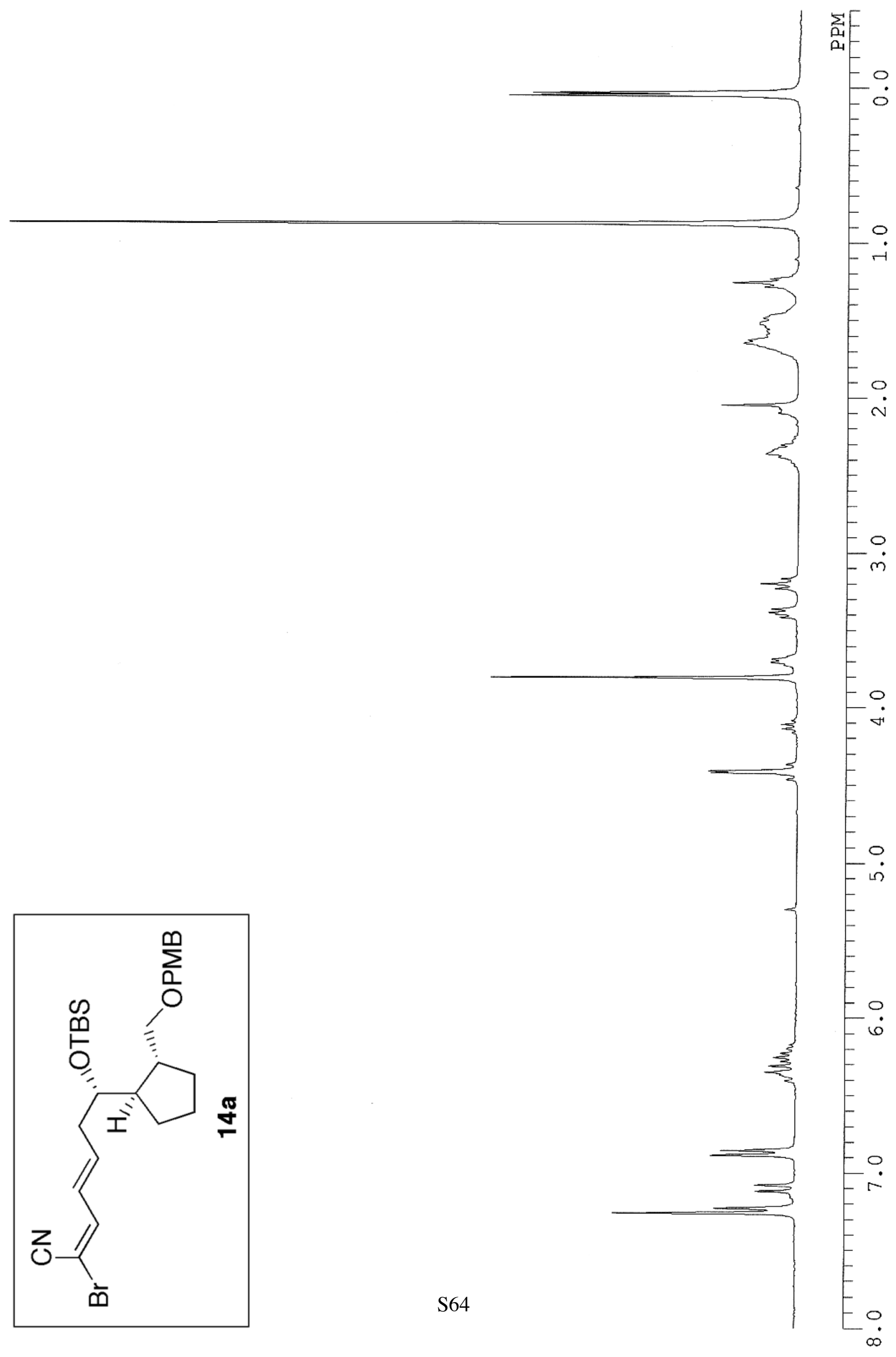




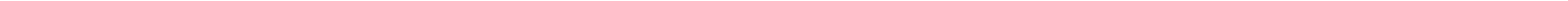




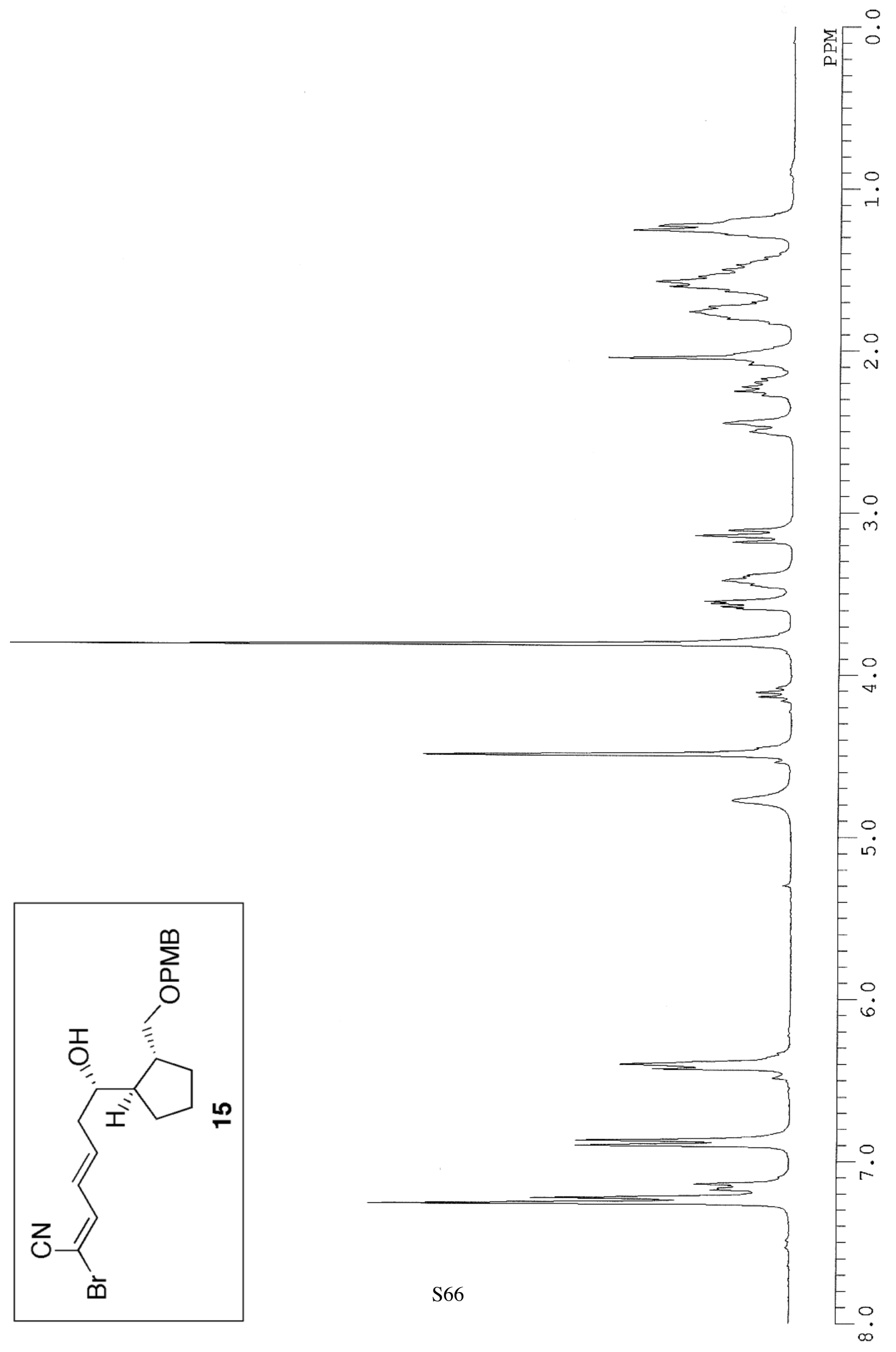




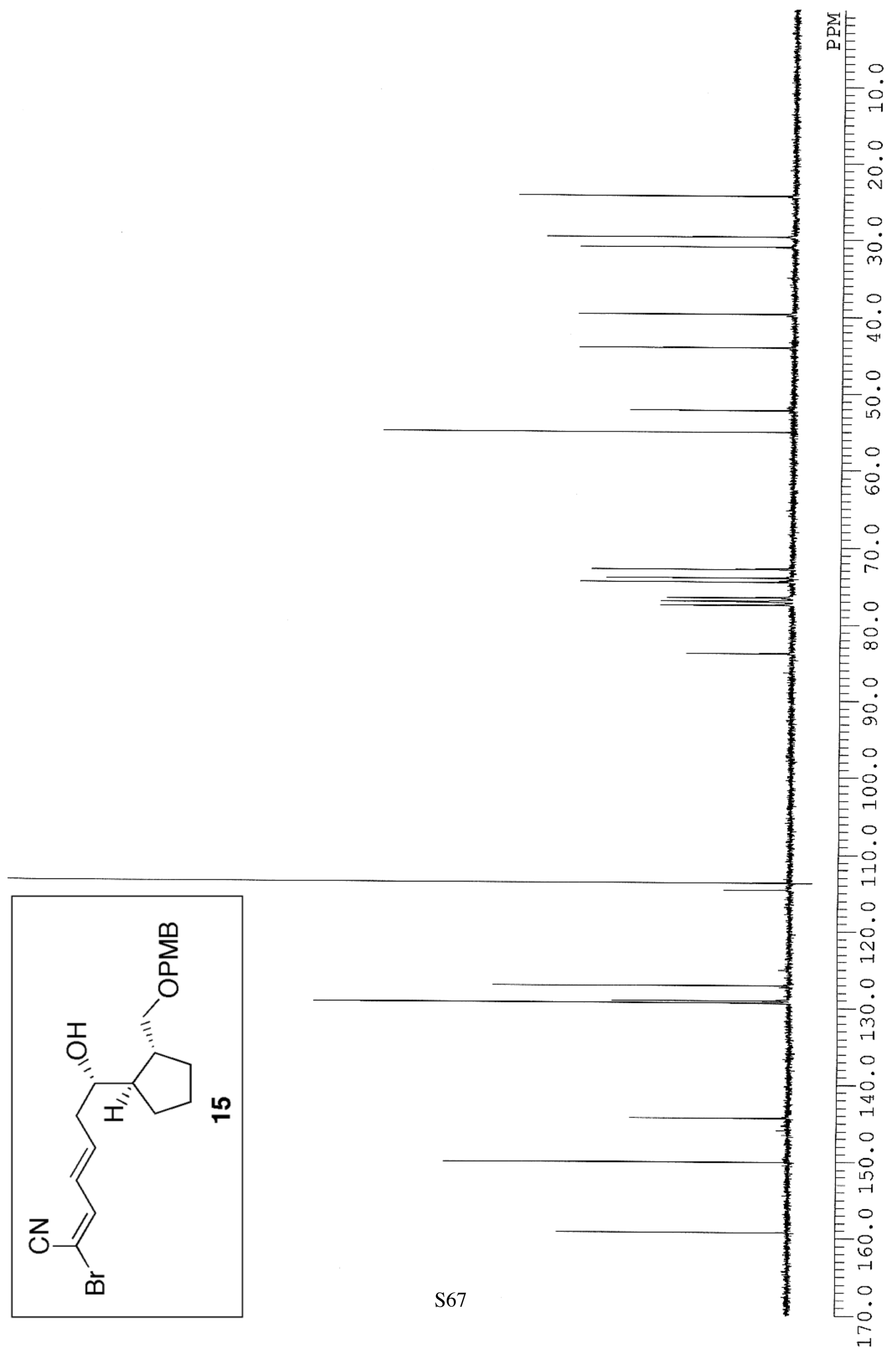




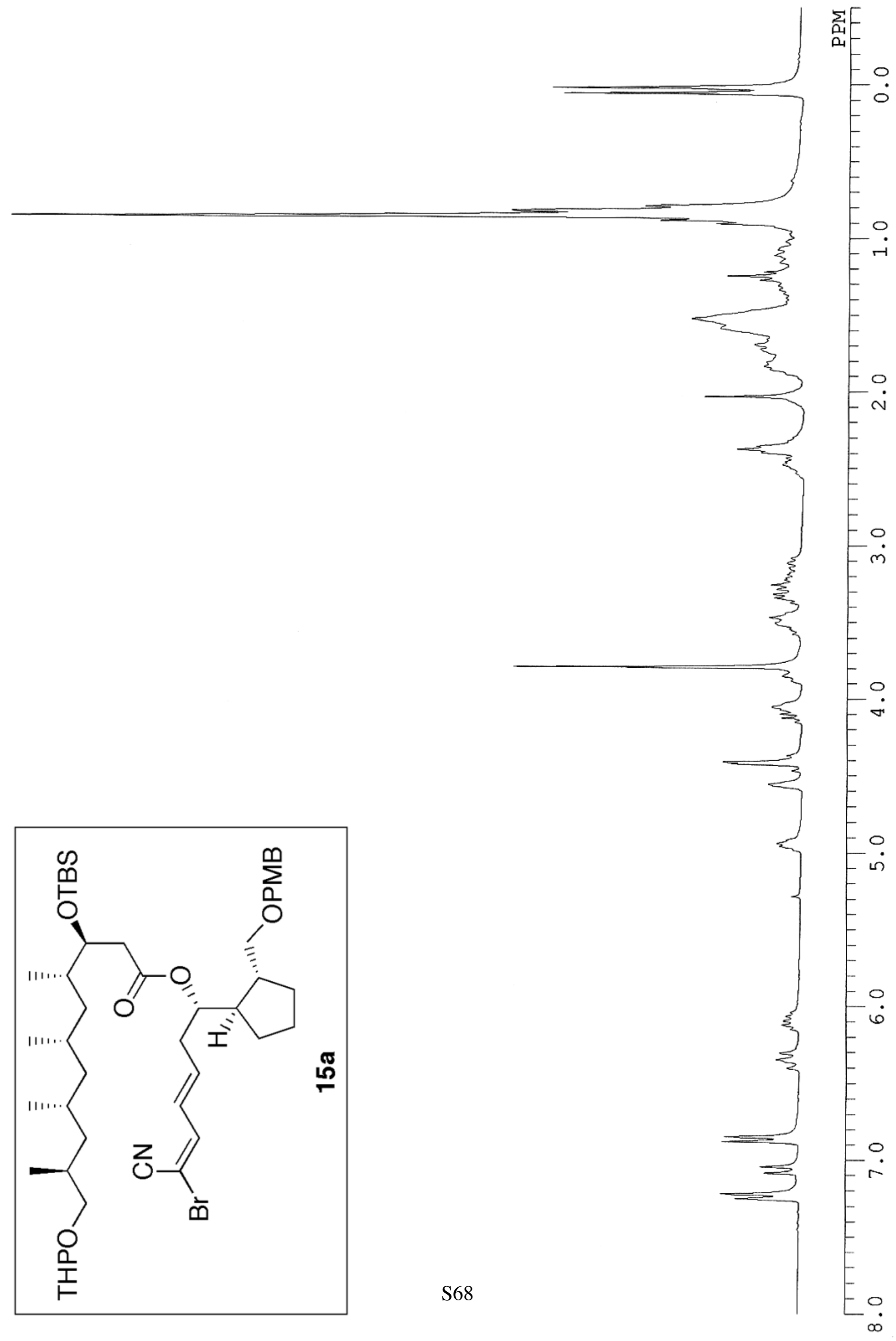




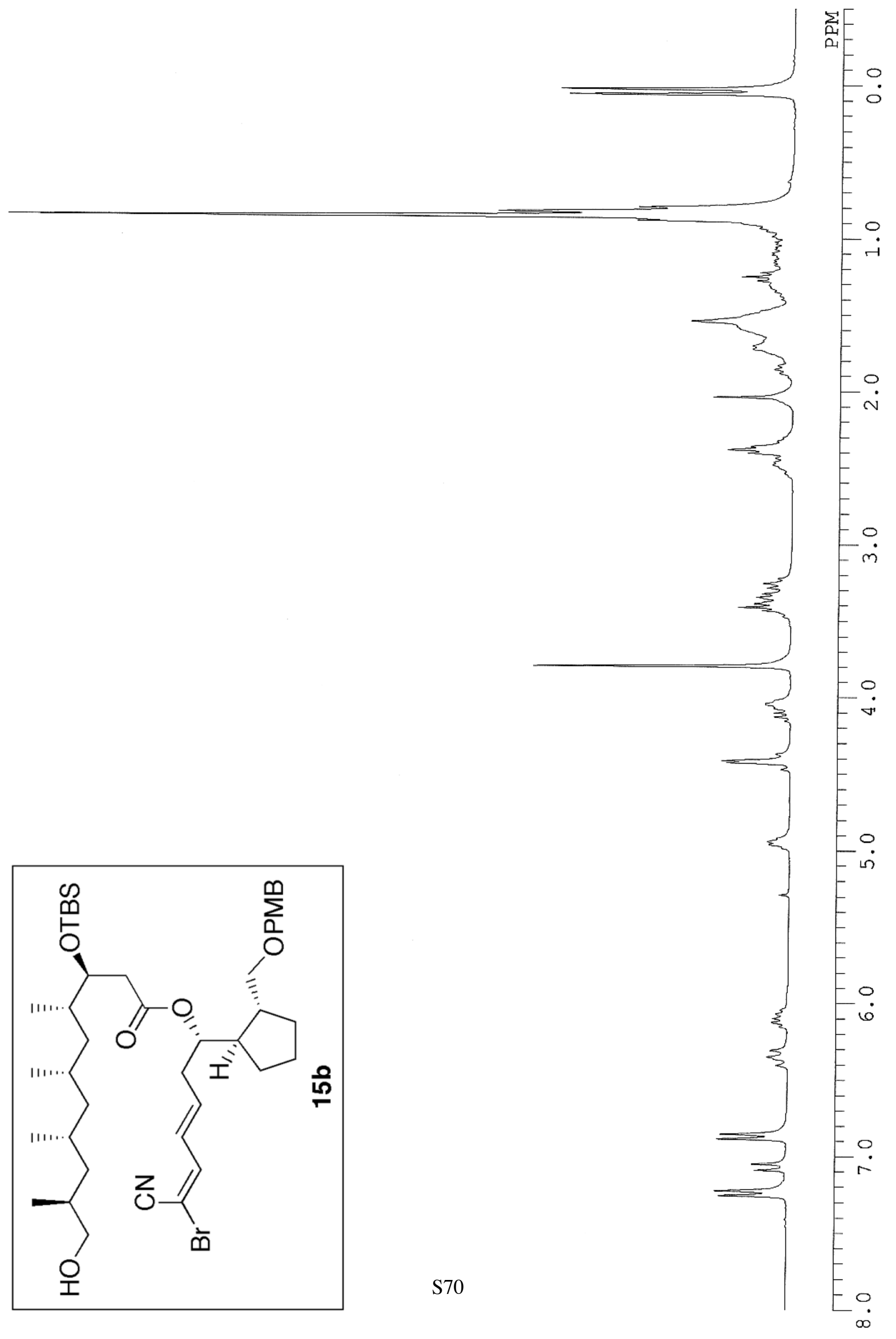




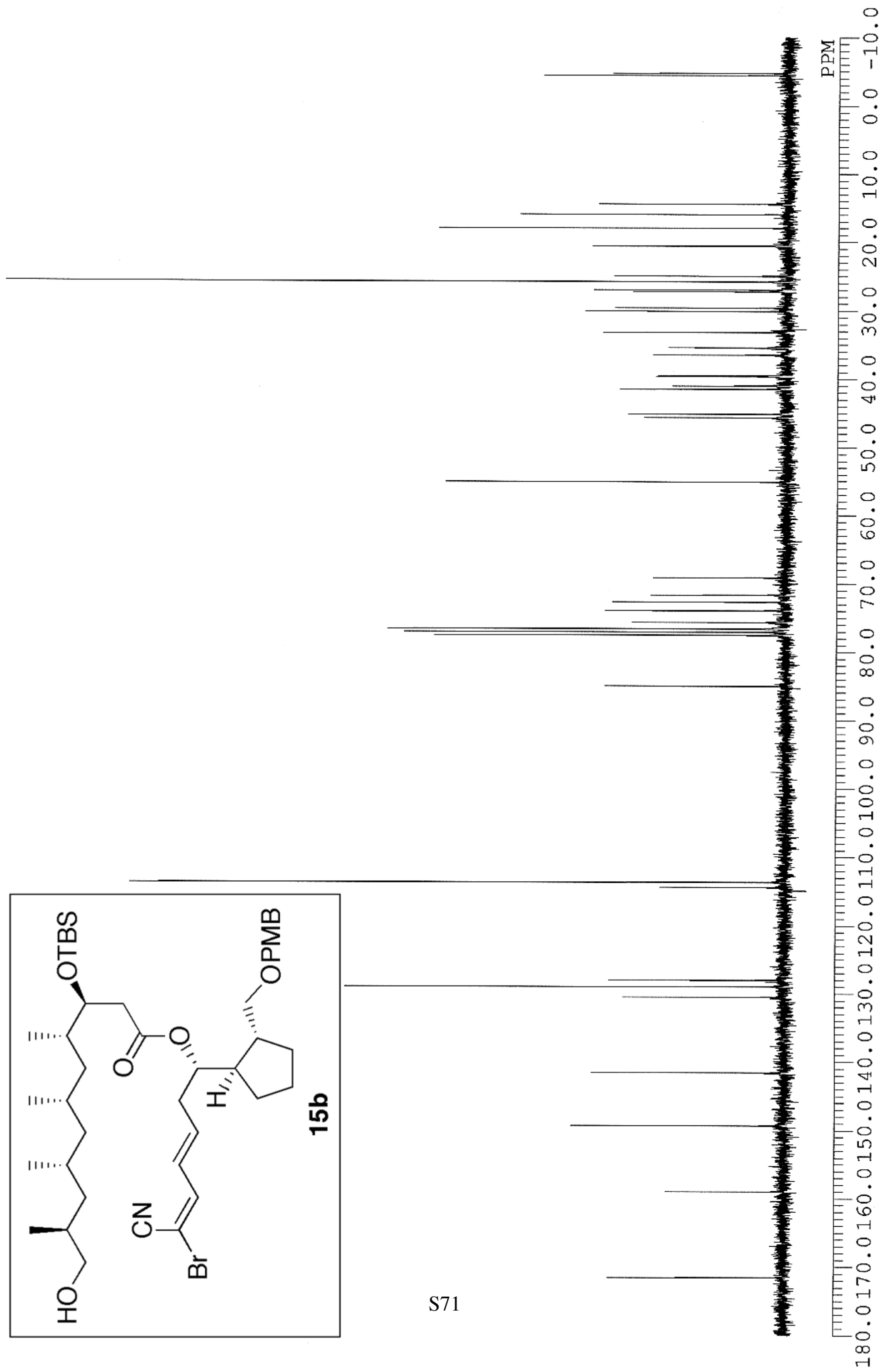




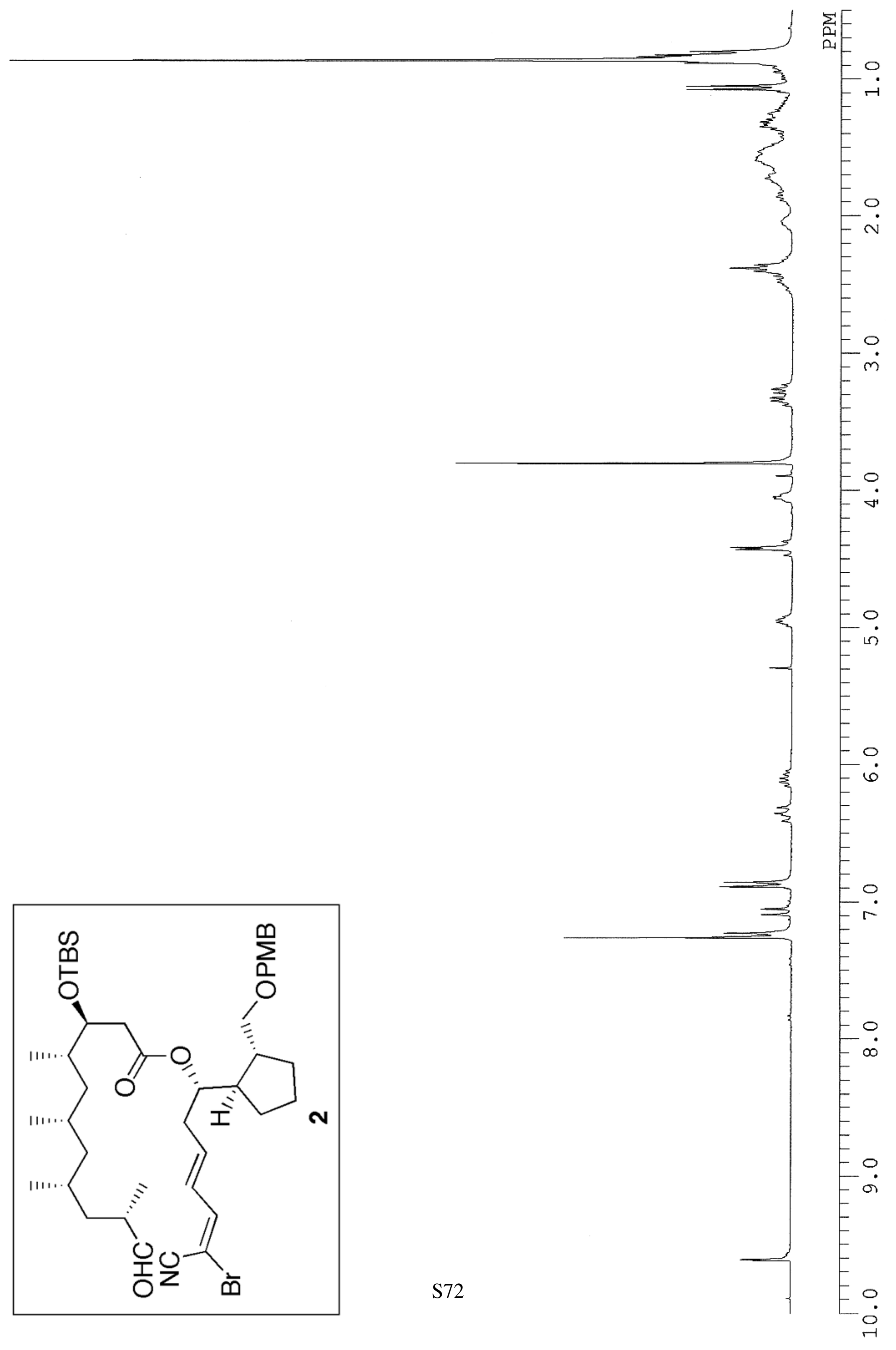




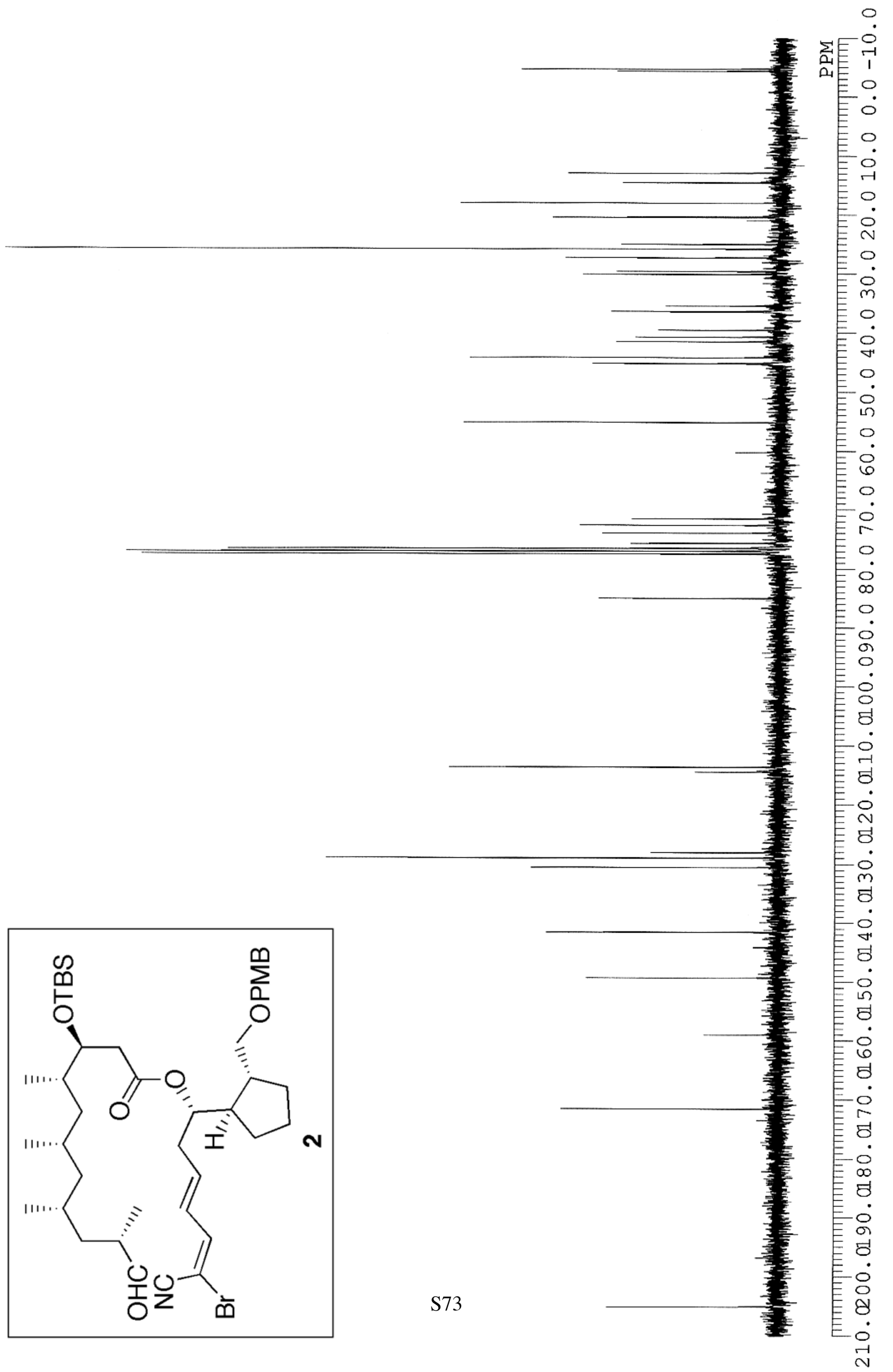




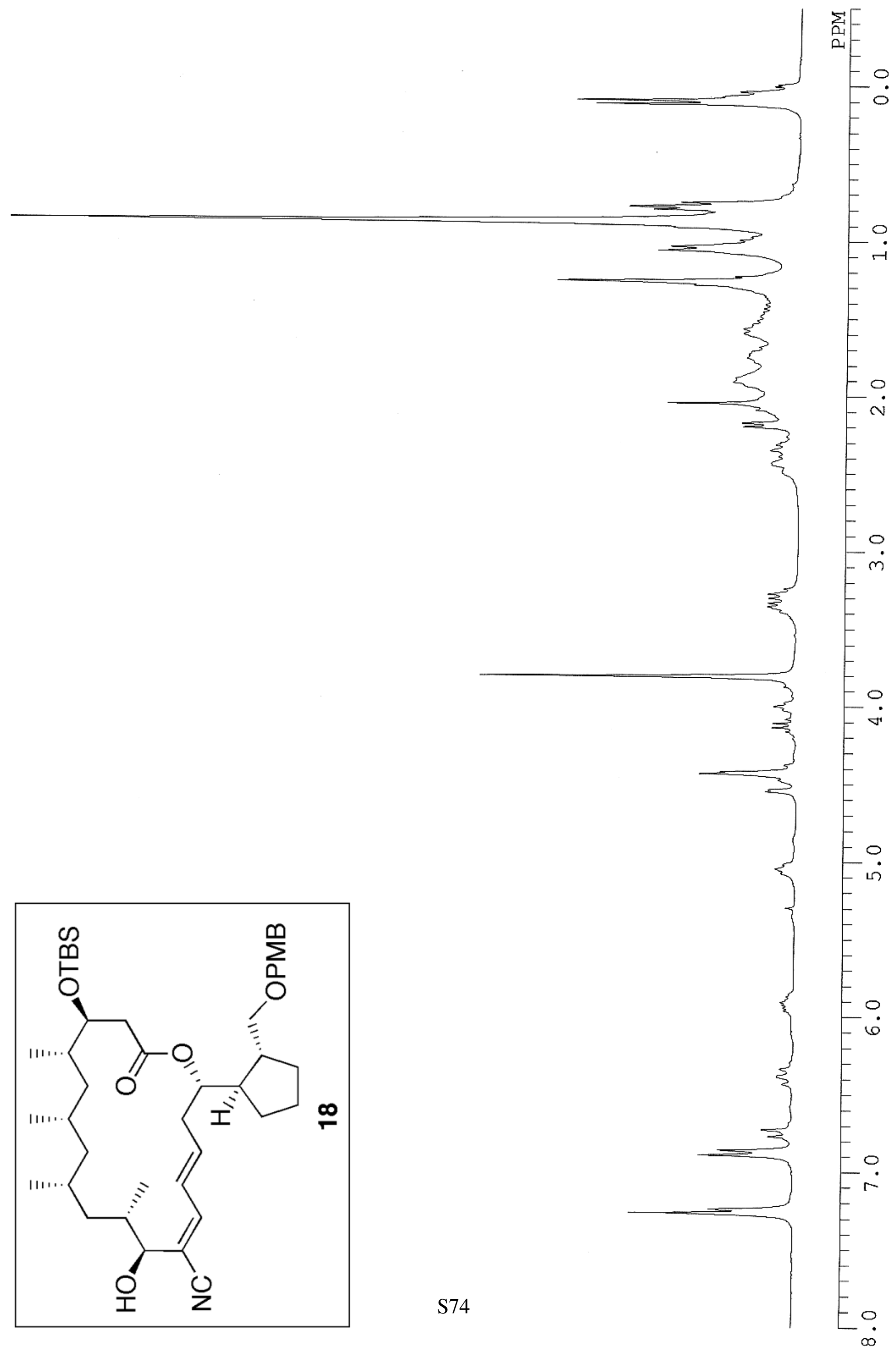




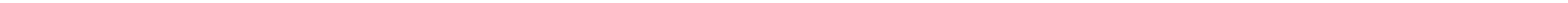




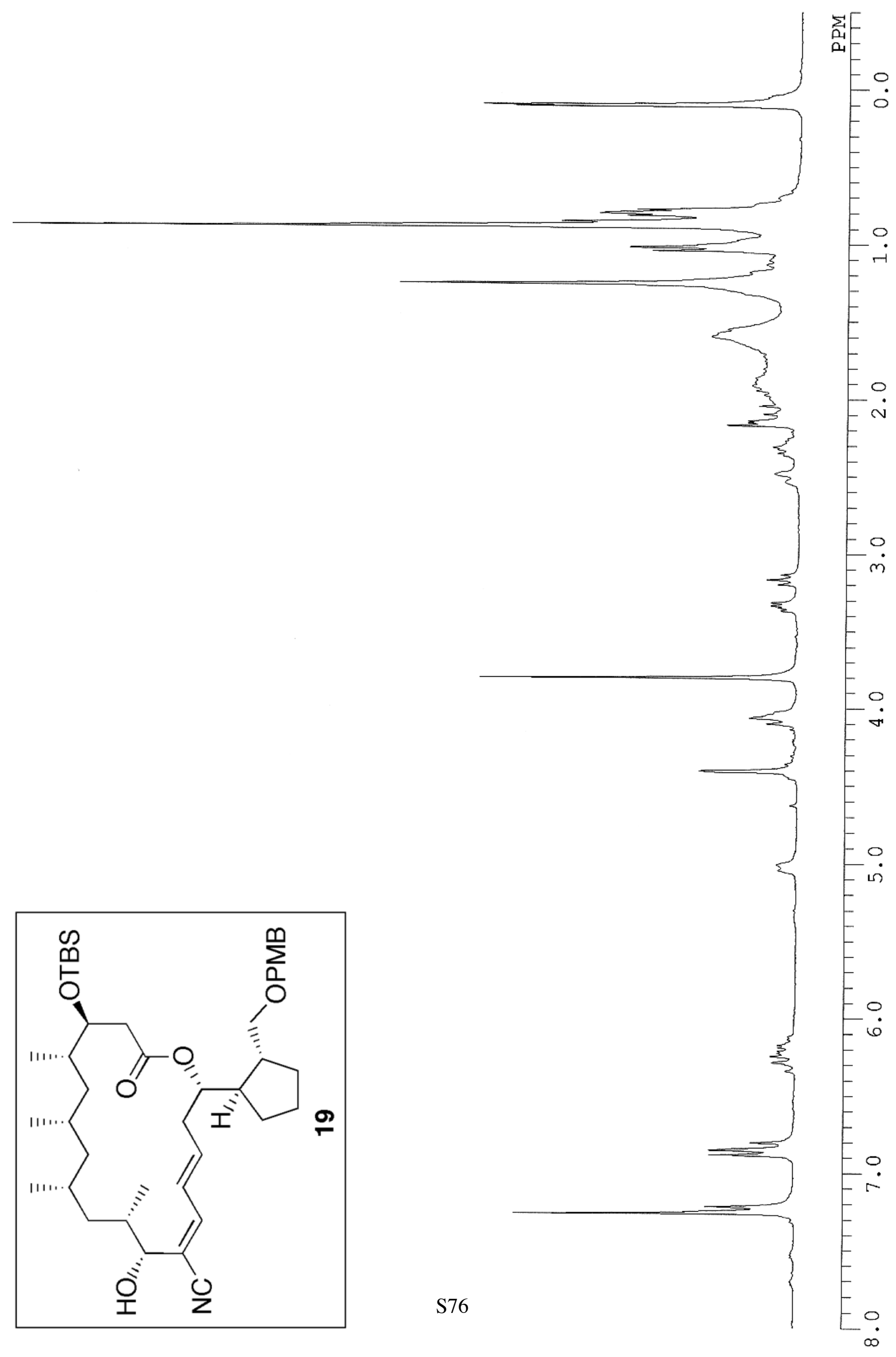




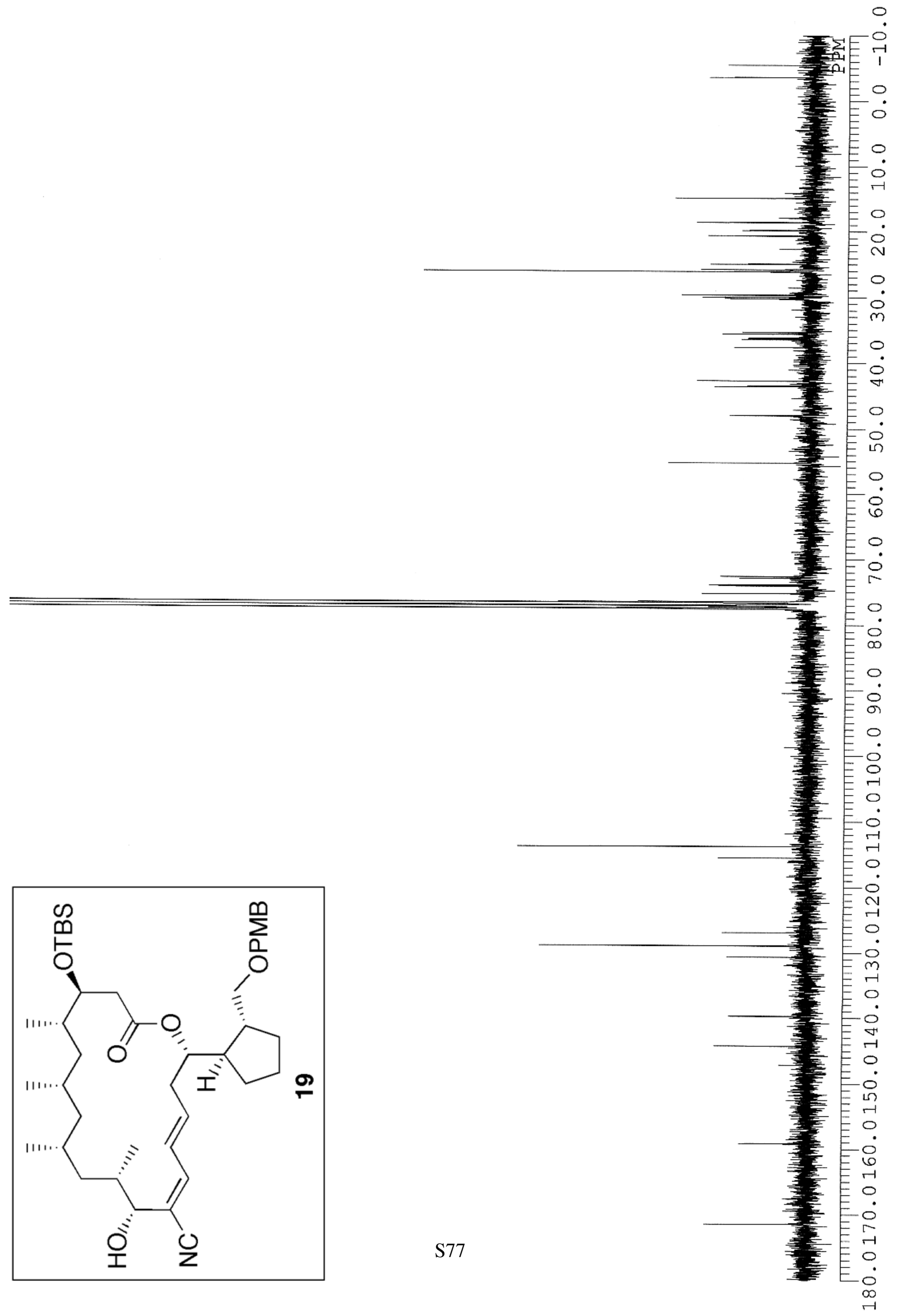




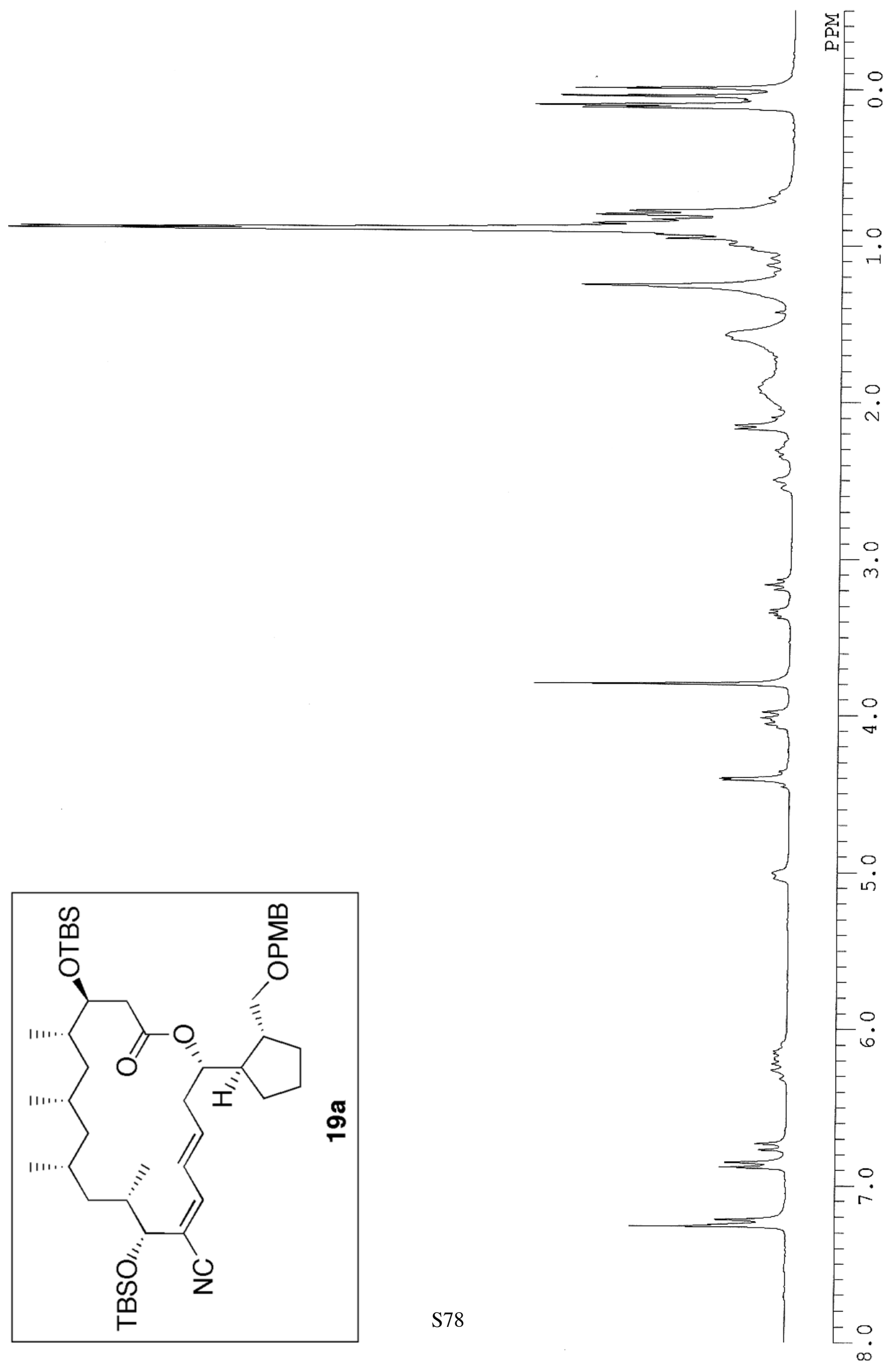




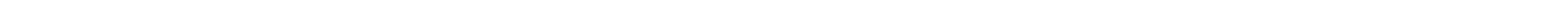




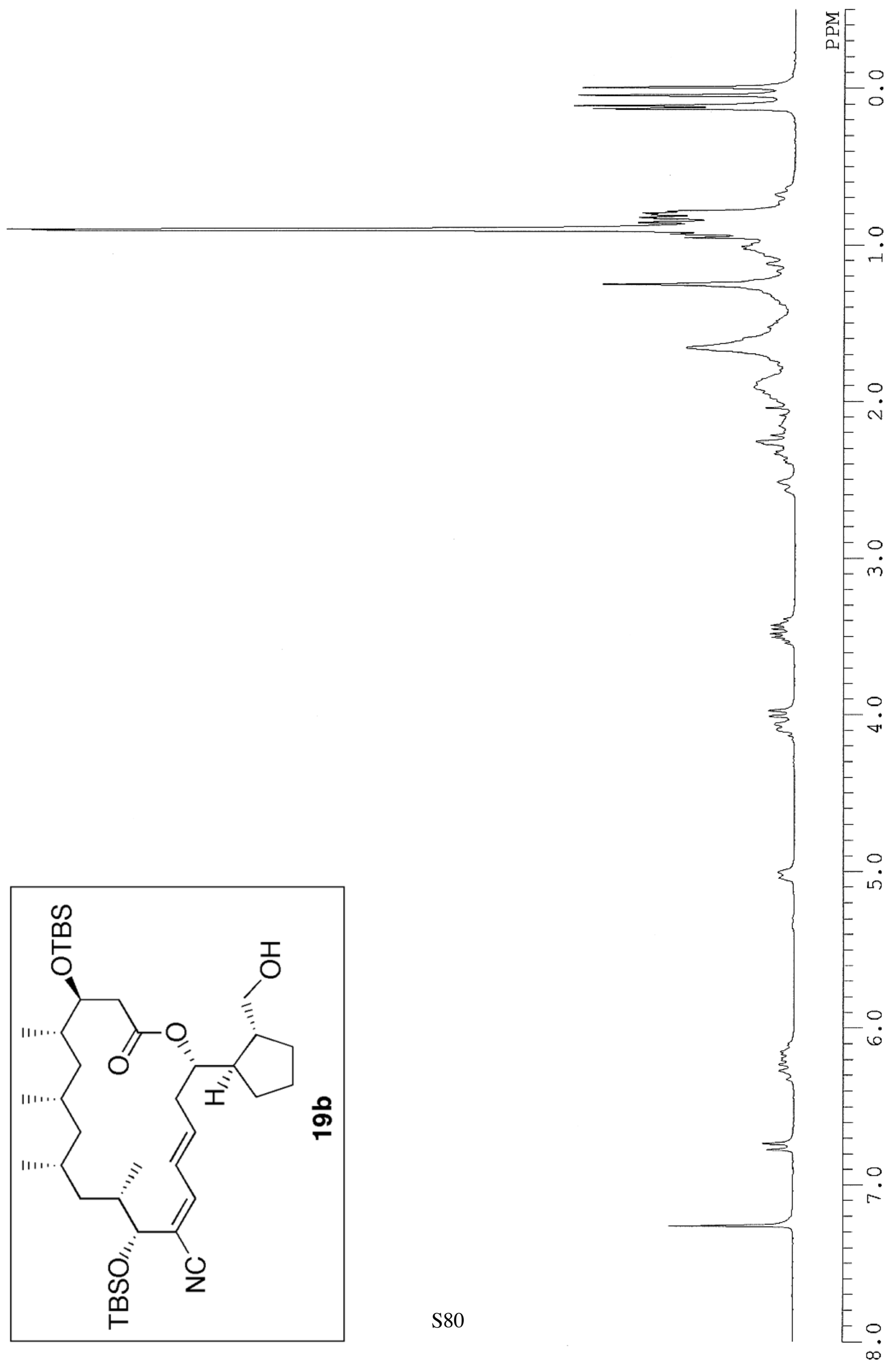




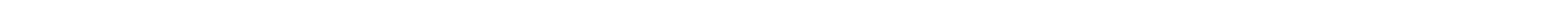




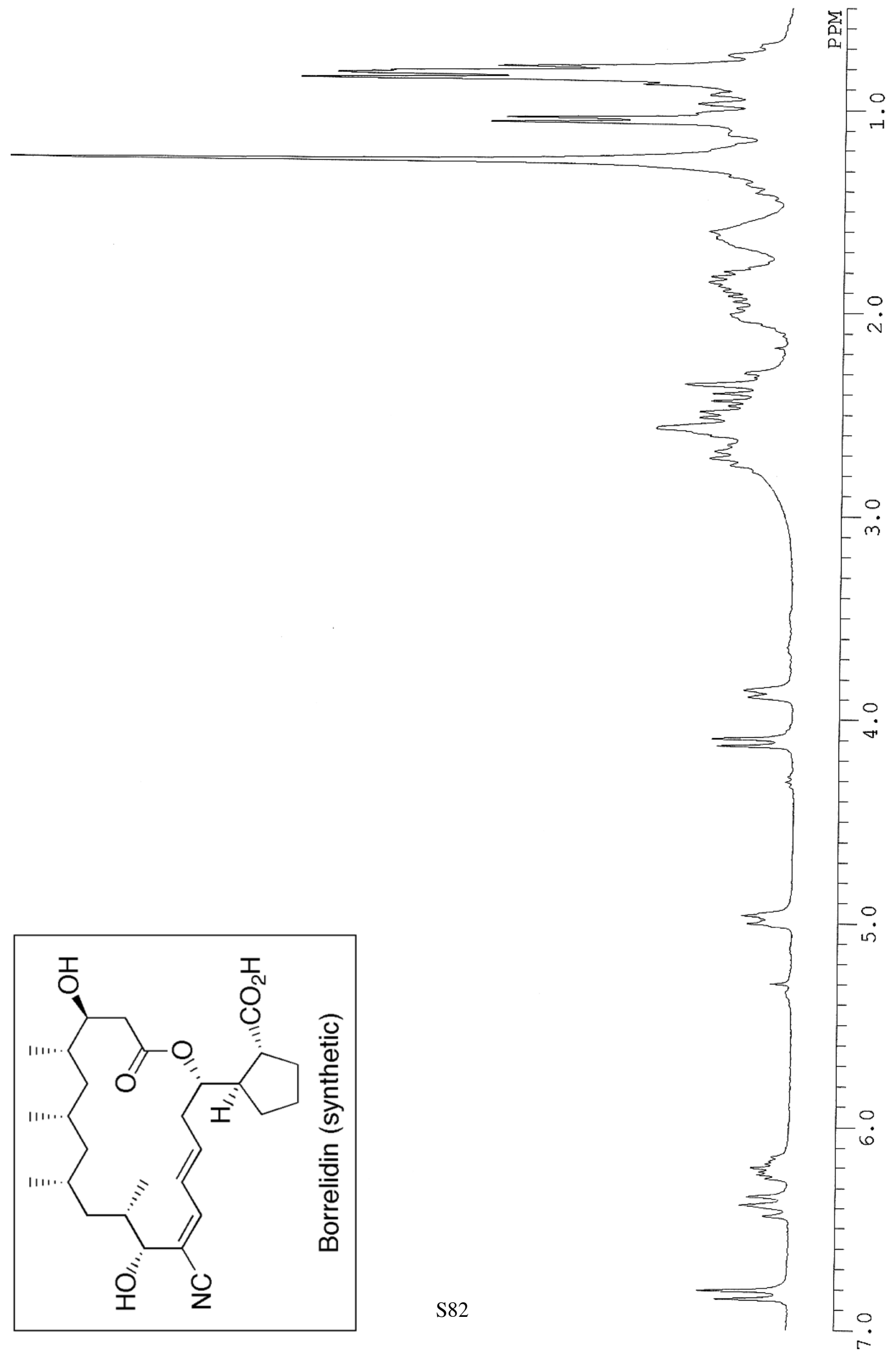




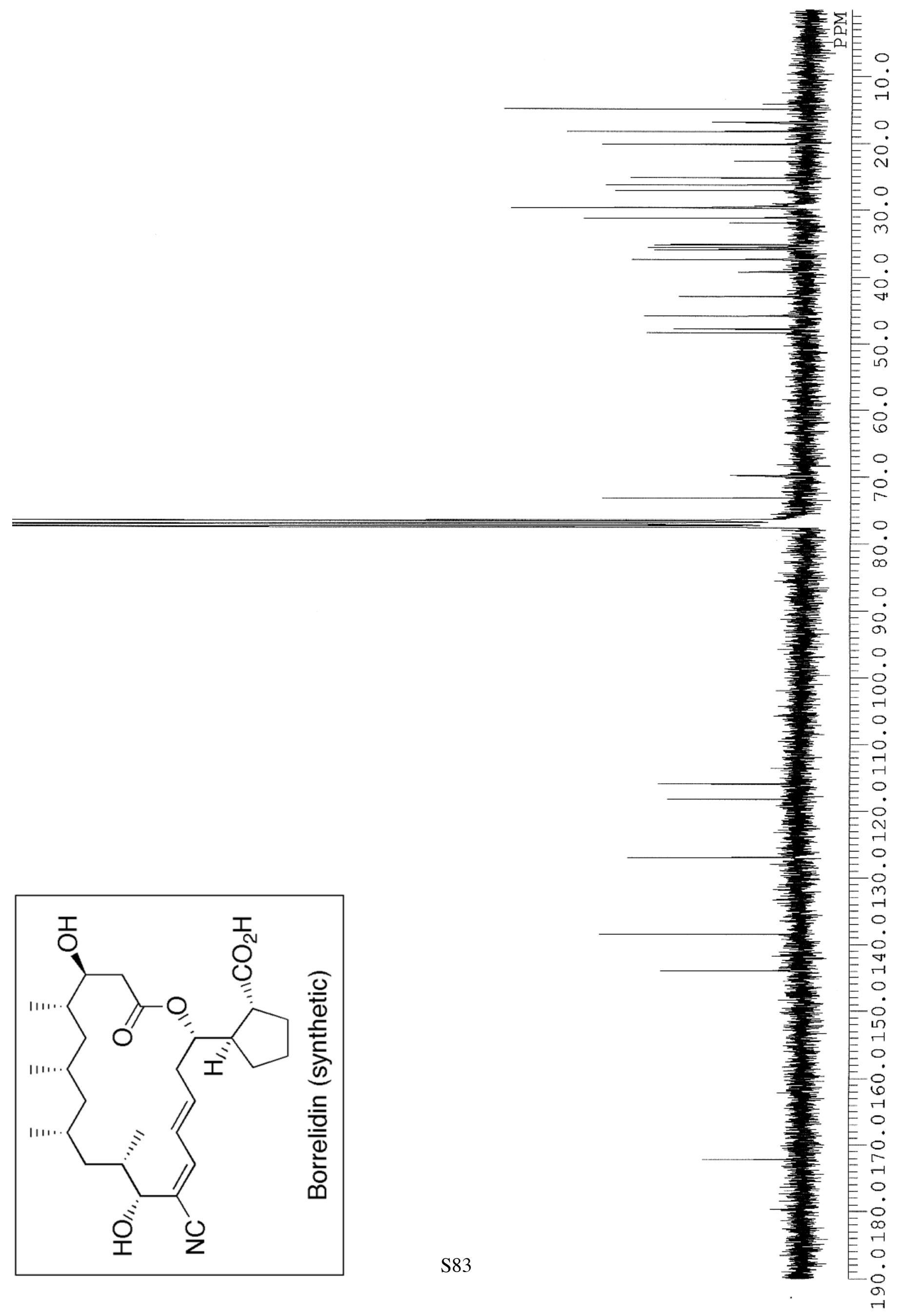




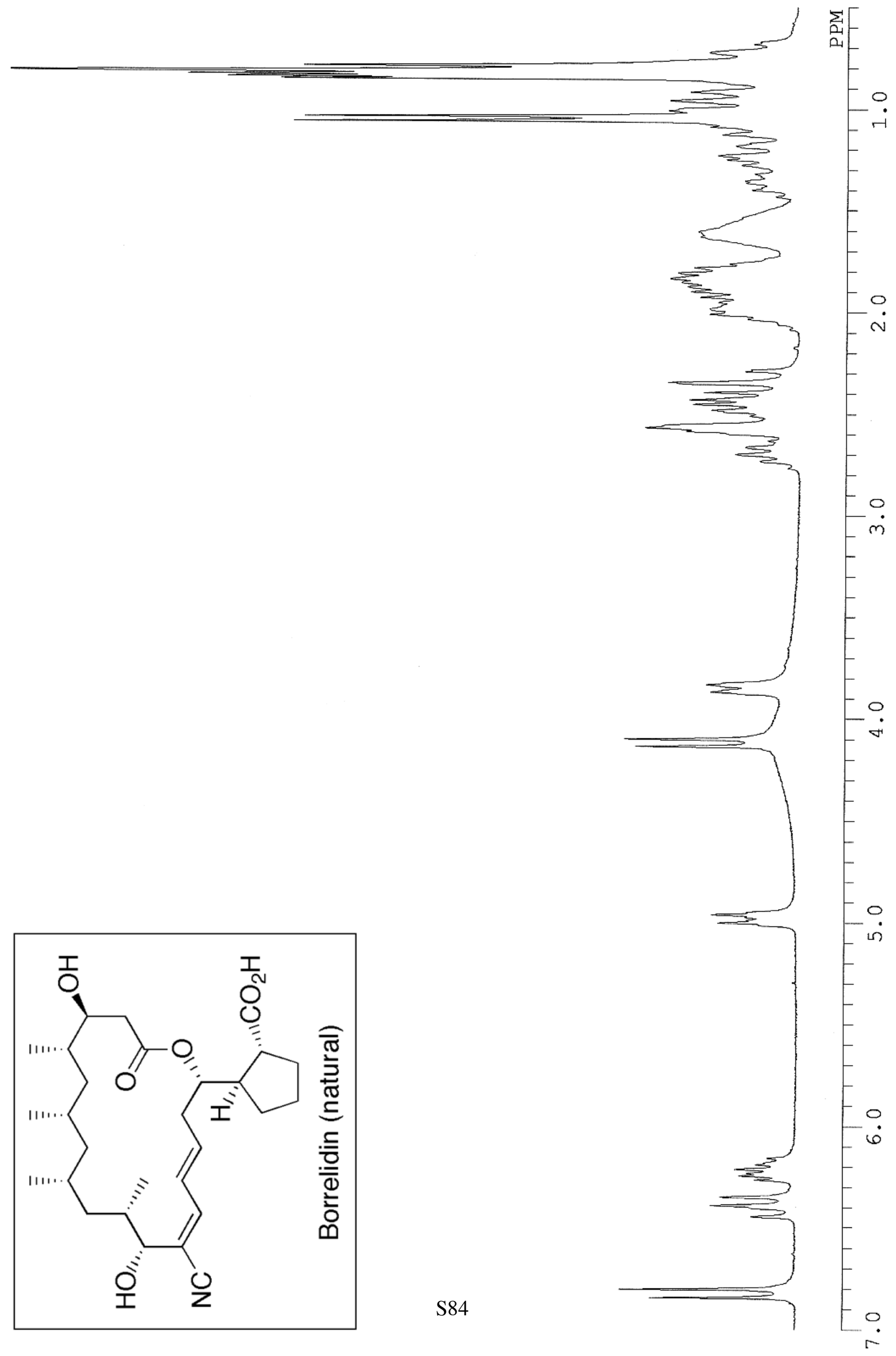




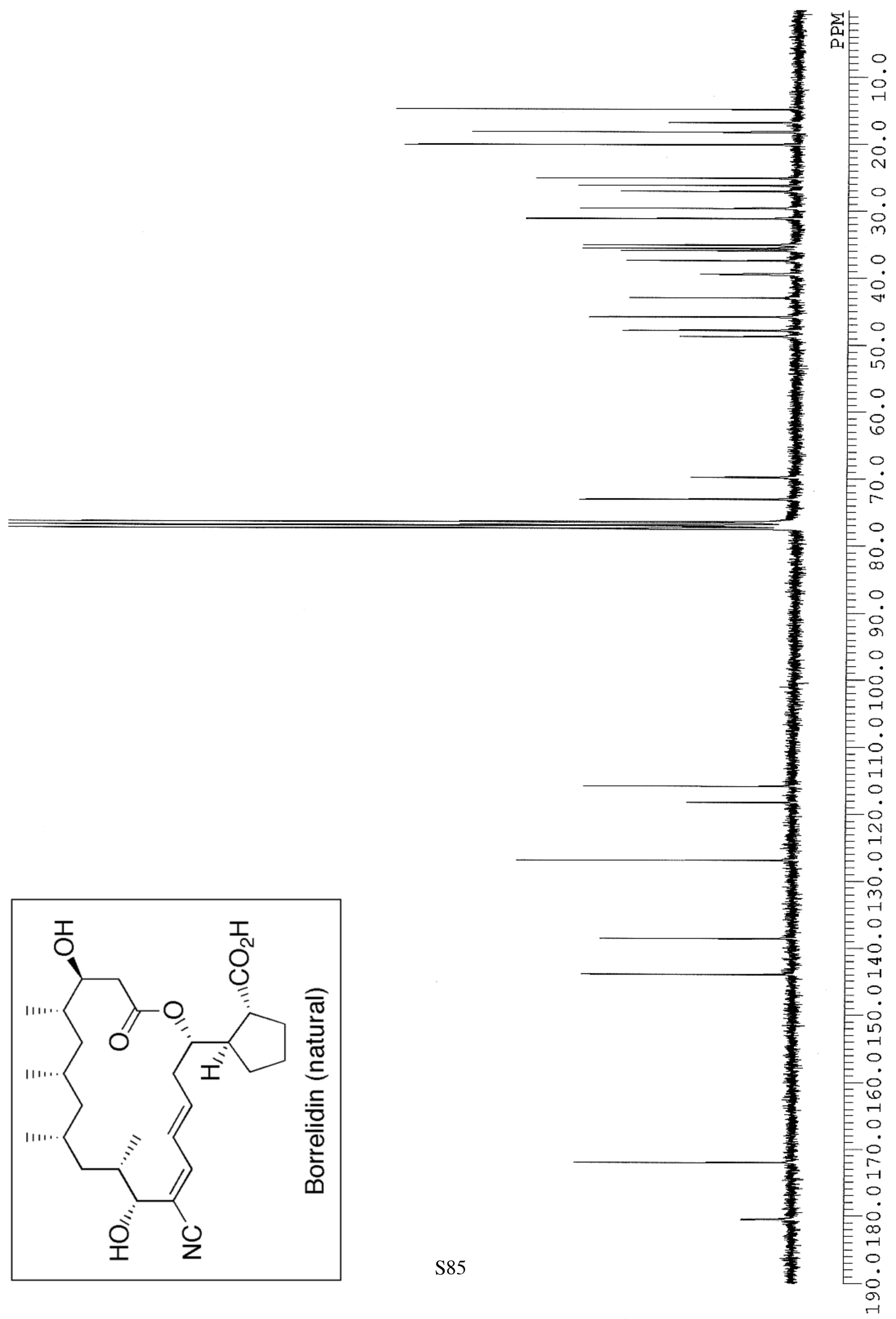

